Departamento de Inteligencia Artificial

Escuela Técnica Superior de Ingenieros Informáticos

\title{
A new agent-based platform for simulating multicellular biocircuits with conjugative plasmids
}

Ph.D. Thesis

Author: Martín Eduardo Gutiérrez Pescarmona, MSc.

Supervisor: Dr. Alfonso Vicente Rodríguez-Patón Aradas 
Dedicated to the memory of mi abuela Alicia, to the memory of mis abuelos Alfonso y Graciela and to my family. 


\section{Acknowledgements}

So, yeah, acknowledgements... So many people to thank, so little space!!! Let's get started:

\section{Spain and family}

First and foremost, I would like to thank my advisor, Alfonso, for bringing me to his lab, supporting my work during these years and teaching me how to research. Without him, none of this could have been possible: Gracias Profe!!!

My family, a multicellular distributed circuit: Gracias Papi por creer en mi y darme consejos de cómo seguir adelante. Tu apoyo ha sido vital en todo el proceso. Gracias Mami y Redro por estar pendientes de mi y de cómo voy y mandarme todos los días un mensaje para saludarme. Los quiero mucho.

Next, my brothers and sisters in arms... my colleagues in the lab. Willy, Pablo, Paula, Vishal (gooooo guys! We're almost done!), Ismael (the Profeta), Marcos, Guillermo, Iván, Luis, Sandra, Irene, Jesús, Angel, Ismael, Aitor, David. You guys were one of the pillars behind this experience and my friends. I have seen you almost everyday at one stage or the other of this journey, and made it a great one. I admire you all, and hope our paths cross again sooner than later. Also my brothers in arms who finished their work in the lab when I was a n00b (still am) in this lab: Jesús and Iñaki. You guys were the role model I wanted to follow when I grew up in the lab. I hope I can live up to the expectation.

A very very special acknowledgement for a big brother who has been with me during the whole duration of my time in Spain, and even now that he is not physically here, still helps me and watches over me. José María: you, Isa, Celia, Silvia and Hyoga made me feel at home here. There are not enough words to thank you for it. I love you guys dearly.

My good friends from UPM: Olga, Bojan, Nina, Luis, Gema, Laura, Freddy, Iñaki, Gherardo, Alberto, Sergio, Ana and Marco. I enjoyed every minute I spent with each and every one of you, I wish you all the best and that we continue to stay in touch for a very long time.

I wish to thank my homies from GMadrid also. Everyday, two hours spent with such great people as yourselves. You arrived in a difficult time of my life and helped me pull through. Thank you for being awesome! 
Arantxa, thank you for your love and support. You have made the last part of this endeavor what I needed it to be. Thank you for being there, being with me and being you. Te quiero mucho!

Also, an acknowledgement to project PLASWIRES for economic support and providing a baseline for the work on this thesis.

Let's now fly (or swim) $11000 \mathrm{kms}$. across the ocean... Chile

Piro, thank you for being by my side for many years. Thank you for your time and love. Your company and support was crucial in this work. I also feel grateful for your family, who supported me in many ways during years.

My friends at UDP. My colleague teachers: Luciano, Jonathan, Diego, Nico, Jorge, Xime and Bea, and all of my ex-students. I love the place! Wait for me! I'll be back...

All of my teachers and friends at PUC, especially Cristian, Carla, Christian, Jorge, Yadran, Jaime and Sole. You are key people in many aspects of my life, and now I would like to follow your example.

Fede, Claudia and Juan Pablo, you were our family during the first year in Madrid. Thank you for watching over us during this time and for sharing a wonderful time with us.

Lucho, George and Mauro: hace 20 años que nos conocemos y Uds. son quizás lo más cercano que tengo a amigos verdaderos de la infancia que me han acompañado en las buenas y en las malas. Los quiero mucho... nunca cambien.

All of my friends and ex-students in Chile. This goes to you: Thank you for trusting that I could achieve this! And to those who did not think I could... you've just made me happier.

I would also like to thank CONICYT, as my studies would have been impossible without their support.

Now into the source code realm... Thank you gro! We had some tough times together, but yeah... I guess that's how our relationship is. Thanks for simulating... my life? I guess...

Pretoriano and Tito Fernández. You guys are still you. I might have mentioned you less in the past few years, but you are the foundations. Never forget that. Thank you for making me me.

Finally, I wish to thank anyone who is not listed here individually and should be. If you are reading this, you definitely deserve to be in this page. 


\section{Contents}

\begin{tabular}{ll}
\hline Contents & 4 \\
\hline
\end{tabular}

\begin{tabular}{ll}
\hline List of Figures & 7
\end{tabular}

$\begin{array}{lc}\text { List of Tables } & 8\end{array}$

\begin{tabular}{lr}
\hline List of Algorithms & 9
\end{tabular}

\begin{tabular}{lll}
\hline & Introduction & 16
\end{tabular}

\begin{tabular}{|lll}
2 & State of the art & 19
\end{tabular}

2.1 Synthetic biology . . . . . . . . . . . . . . . . . . . . . . . . . . 19

2.1 .1 Gene Expression . . . . . . . . . . . . . . . . . . 20

2.1 .2 Gene Regulatory Networks _. . . . . . . . . . . . . . 22

2.1 .3 Network motifs . . . . . . . . . . . . . . . . . . . . . . 24

2.1 .4 Intercellular communication . . . . . . . . . . . . . . 25

2.2 Multicellular circuits . . . . . . . . . . . . . . . . . 28

2.3 Temporal and spatio-temporal pattern circuits . . . . . . . . . 29

$2.3 .1 \quad$ Bullseye pattern $\ldots \ldots \ldots \ldots \ldots \ldots$

2.3 .2 Edge detector $\ldots \ldots \ldots \ldots \ldots$

2.3 .3 French flag model . . . . . . . . . . . . . . . . . . 31

2.4 AbM simulators . . . . . . . . . . . . . . . . . . . . . 32

$2.4 .1 \quad$ Krone et al. Interacting Particle System Model . . . . . . 32

2.4 .2 BactoSim . . . . . . . . . . . . . . . . 33

2.4 .3 DiSCUS $\ldots \ldots \ldots \ldots \ldots \ldots$

2.4 .4 iDynoMiCS . . . . . . . . . . . . . . . . . . . . 34

2.4 .5 CellModeller . . . . . . . . . . . . . . . . . . 35

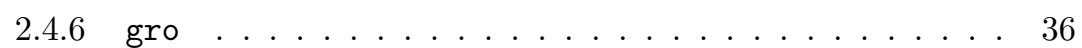

2.4 .7 Other AbM simulators . . . . . . . . . . . . . . . . . 37

2.4 .8 Programming frameworks for implementing AbMs . . . . 37

\begin{tabular}{lll}
\hline 3 & Problem definition & 38
\end{tabular}

3.1 Motivation . . . . . . . . . . . . . . . . . . . . . 38

3.2 Research questions . . . . . . . . . . . . . . . . . . . . . . 39

3.3 Methodology $\ldots \ldots \ldots \ldots$. . . . . . . . . . . . . . 4 41 
4 Proposed solution 44

4.1 The first version of gro . . . . . . . . . . . . . . . . 44

4.2 A new version of gro . . . . . . . . . . . . . . . . . 45

$4.2 .1 \quad$ Improving physical engine speed: from Chipmunk to Cel-

lEngine $\ldots \ldots \ldots \ldots \ldots \ldots \ldots \ldots$

4.2.2 Enhancing cell programmability: from guarded commands to CellPro with ProSpec . . . . . ........ 51

4.2 .3 Extending intercellular communication: Conjugation and signals $\ldots \ldots \ldots \ldots \ldots \ldots \ldots \ldots \ldots$

$4.2 .4 \quad$ Nutrients: CellNutrient $\ldots \ldots \ldots \ldots$

4.3 Novel multicellular circuits $\ldots \ldots \ldots \ldots$. . . . . . . . . . . 65

$4.3 .1 \quad$ Spatio-temporal patterns . . . . . . . . . . . . . . 65

4.3 .2 Temporal patterns $\ldots \ldots \ldots \ldots$. . . . . . . . . . . 76

4.4 Discussion $\ldots \ldots \ldots \ldots \ldots \ldots \ldots$

$4.4 .1 \quad$ CellEngine $\ldots \ldots \ldots \ldots$

$4.4 .2 \quad$ CellPro and ProSpec . . . . . . . . . . . . . . . . . . . . 79

$4.4 .3 \quad$ Bacterial Conjugation . . . . . . . . . . . . . . . . . . 81

$4.4 .4 \quad$ CellSignals $\ldots \ldots \ldots \ldots$. . . . . . . . . . . . . . . 81

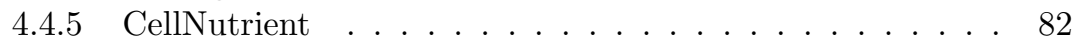

4.4 .6 Multicellular circuits . . . . . . . . . . . . . . . . . 82

5 Conclusions and future work $\quad 84$

5.1 Conclusions . . . . . . . . . . . . . . . . . 84

5.2 Future work . . . . . . . . . . . . . . . . . . 86

\begin{tabular}{ll}
\hline Bibliography & 87
\end{tabular}

\begin{tabular}{ll}
\hline A ProSpec specification reference & 100
\end{tabular}

A.1 A GRO specification (with its new syntax) . . . . . . . . . 100

A.1.1 Library inclusion, parameter setting, definition of global variables and values $\ldots \ldots \ldots \ldots \ldots$. . . . . . . . . . . . .

A.1.2 Genetic entities and their relationships. . . . . . . . . 102

A.1.3 Actions associated to protein conditions . . . . . . . . . . 105

A.1.4 Top-level programs and global control . . . . . . . . . . 108

A.2 Basic specification examples . . . . . . . . . . . . . . . . . . . 109

A.2.1 newLtest.gro . . . . . . . . . . . . . . . . . . . . . . . . 109

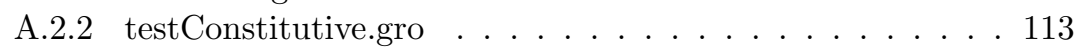

\begin{tabular}{ll}
\hline B .gro sources & 117
\end{tabular}

B.1 YES Gate specification with guarded commands . . . . . . . . 117

B.2 YES Gate specification in ProSpec . . . . . . . . . . . . . . . 121

B.3 Two-level bullseye pattern . . . . . . . . . . . . . . . . . . . . . 122

B.4 Three-level bullseye pattern . . . . . . . . . . . . . . . . . . . 124

B.5 Four-level bullseye pattern . . . . . . . . . . . . . . . . . . . . 127

B.6 Spatial oscillator with no initiating switch, variability or noise. . 131

B.7 Spatial oscillator with initiating switch but no variability or noise 133

B.8 Spatial oscillator with no initiating switch having variability and

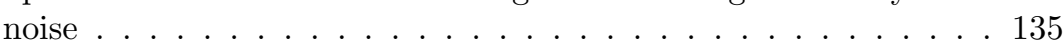

B.9 Spatial oscillator with initiating switch having variability and noise 137

B.10 Nutrient generated sun pattern . . . . . . . . . . . 139 
B.11 Synthetic generated sun pattern. . . . . . . . . . . . . . . . . 140

B.12 Autonomous bioreactor . . . . . . . . . . . . . . . . . . 142 


\section{List of Figures}

$2.1 \quad$ Gene expression process . . . . . . . . . . . . . . . . . . . . . . 22

2.2 Depiction of an operon . . . . . . . . . . . . . . . . . . . . . . 23

2.3 Feed-forward Loops . . . . . . . . . . . . . . . . . . . . . . . . . . . . . . . . . . . . . . . . . . 24

2.4 Lux QS system . . . . . . . . . . . . . . . . . . . . . . . . . . . . . . . . . 26

2.5 Schematization of conjugation . . . . . . . . . . . . . . . . . . . . 28

2.6 Original bullseye pattern by Basu et al. . . . . . . . . . . . . . . . . 30

2.7 Edge detecting circuit by Tabor et al. . . . . . . . . . . . . . . . 31

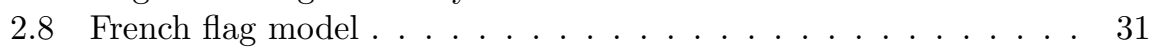

$4.1 \quad$ Original gro architecture . . . . . . . . . . . . . . . . . . . . . 45

4.2 Updated gro architecture . . . . . . . . . . . . . . . . . . . . . . . . . . . . . . . . 46

4.3 New gro workflow . . . . . . . . . . . . . . . . . . . . . . . 46

4.4 Chipmunk shoving dynamics in gro . . . . . . . . . . . . . . . . . . . . . . . 47

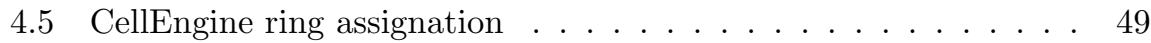

4.6 CellEngine relaxation step . . . . . . . . . . . . . . . . . . . . . . 49

4.7 CellEngine relocation step . . . . . . . . . . . . . . . . . . . . . . . . 50

4.8 gro performance . . . . . . . . . . . . . . . . . 50

4.9 CellEngine validation . . . . . . . . . . . . . . . . . . 51

4.10 Protein expression in CellPro . . . . . . . . . . . . . . . . . . . . . . . . . . . . . . 53

4.11 Simulating bacterial conjugation . . . . . . . . . . . . . . . . 57

4.12 Signal area limit . . . . . . . . . . . . . . . . . . . 59

4.13 Dynamic sized signal grid . . . . . . . . . . . . . . . . . . . . . 60

4.14 Custom integration matrix . . . . . . . . . . . . . . . . . . . 61

4.15 Digital signals . . . . . . . . . . . . . . . . . . . . . . . . . . . . . . . 62

4.16 Colony growth with nutrients . . . . . . . . . . . . . . . 64

4.17 Expected results for bullseye pattern circuits . . . . . . . . . . . 65

4.18 Two-level multicellular bullseye pattern . . . . . . . . . . . . . . 67

4.19 Three-level multicellular bullseye pattern . . . . . . . . . . . . . 69

4.20 Four-level multicellular bullseye pattern . . . . . . . . . . . . . . 70

4.21 Expected results for spatial oscillator pattern circuits . . . . . . . 71

4.22 Spatial oscillators . . . . . . . . . . . . . . . 72

4.23 Spatial oscillators with noise and variability . . . . . . . . . . . 73

4.24 Expected results for sun pattern circuits . . . . . . . . . . . . . . 73

4.25 Sun pattern generated with nutrient consumption . . . . . . . . . 74

4.26 Sun pattern generated with a synthetic circuit . . . . . . . . . . . 75

4.27 Expected sequence for the autonomous bioreactor. . . . . . . . . 76

4.28 Design of the Autonomous Bioreactor . . . . . . . . . . . . . . 77

4.29 Simulation of the Autonomous Bioreactor . . . . . . . . . . . . . 78 


\section{List of Tables}

$2.1 \quad$ FFL functions . . . . . . . . . . . . . . . . . 25 


\section{List of Algorithms}

4.1 Bacterial conjugation . . . . . . . . . . . . . . 57

4.2 Nutrient uptake $\ldots \ldots \ldots \ldots$. . . . . . . . . . . . . . 63 


\section{Abstract}

Synthetic biology is a recently born discipline, finding its origins in the year 2000 at the MIT Artificial Intelligence lab. It emerges as a discipline in which engineering is applied to biological components and systems. As per its nature, synthetic biology is an interdisciplinary field in which engineers and biologists work together to design and implement circuits that either seek to recreate life or display an unnatural behavior for a specific purpose. Until recently, this engineering of biology has been carried out in an informal manner, as bioCAD and simulation tools were not available to aid in the design of biocircuits. A trial-and-error cycle along with the biologist intuition are the guidelines for a long process in reaching the desired biocircuit behavior. This places synthetic biology at a stage that can be compared to microelectronics in the 1980s. Trialand-error is a slow method, simulators and bioCAD tools are a requirement for speeding up the process. The design-build-test-learn cycle is the approach most synthetic biologists currently use to undertake the task. This cycle takes full advantage of the software tools to speed up the process. In the design phase of this cycle, the scientist works on a design and elaborates on its functionality in a theoretical manner. It is at this stage that computer simulations become useful, as they reproduce the basic dynamics of the designs. These programs serve the purpose of assisting in identifying flaws and ruling out incorrect designs. They also help in directing and estimating parameters to implement designs in the wet lab. One goal is to minimize the costs in time and money due to experimentation. In this scenario, there is a clear need for fast, accurate and simple to use simulators.

Agent based Models (AbMs), also known as Individual based Models (IbMs), are a type of models implemented by simulators that focus on the behavior of each individual in a colony (in this context, it is a colony of cells). These models are alternatives to Ordinary Differential Equation (ODE) or stochastic (Gillespie) based models. AbMs provide a better representation of how bacterial populations interact and behave in a spatial manner, whilst ODE and Gillespie models offer a more accurate simulation of dynamics at an intracellular level. Bacterial colonies grow in a Petri dish in a 2D manner. Each bacterium can be seen as an individual agent that communicates and interacts with other bacteria through environmental chemical signals or by exchanging genetic messages (plasmids). Each cell follows probabilistic rules that direct its growth, reproduction, communication, death, or any action it executes. This accounts for the whole bacterium behavior. These probabilistic rules yield the emergence of a global population-level behavior. AbM simulators are well-suited for assessing and predicting multicellular synthetic circuit behavior. 
This leads to three research questions on the matter: 1) What potential do current AbM simulators have for simulating novel multicellular genetic circuits using bacterial conjugation? 2) How should the identified shortcomings be overcome to enable the simulation of novel multicellular genetic circuits using bacterial conjugation? 3) What novel designs relating to spatial and/or temporal patterns can the newly enhanced platform prototype?

When this work began, no AbM simulator was capable of simulating multicellular circuits in the PLASWIRES project. gro was selected as the AbM framework to be improved and extended. Following this choice, and the scaling complexity in simulations, a roadmap of features to complement it was established:

- Improving the simulator speed: this was done by implementing CellEngine, a new physics engine featuring a shoving algorithm tailored for bacterial colonies.

- Adding bacterial conjugation as an intercell communication mechanism: implemented directly into the gro source code, being a key requirement for simulating PLASWIRES circuits.

- Simplifying the gene expression process in the simulator and simulation specification: CellPro is a new module that simplifies gene expression through binary protein dynamics. ProSpec defines a new scalable specification language for describing complex genetic circuits.

- Adding nutrient uptake and modulating cell growth: a Monod based nutrient uptake simulator module, CellNutrient, was developed to couple with gro.

- Improving the environmental signal capabilities: CellSignals packages a new and extended version of environmental signal simulation for gro.

gro was improved substiantially upon inclusion of the proposed features. It is now capable of simulating $10^{5}$ bacteria in a matter of minutes (instead of a week). This newly-acquired speed of the simulator places it as a very fast prototyping tool for a wide range of systems and synthetic biology designs. Additionally, gro now provides built-in genetic specification and dynamics, nutrient consumption, improved environmental signal capabilities and bacterial conjugation. This platform, along with the mentioned modules are open-source and can be found at: https://github.com/liaupm/GRO-LIA. Realism of the simulations produced by the platform has increased as high cell counts are now attainable and can make use of the new features. Outcomes of these simulations accurately shed light on the behavior of multicellular circuits, fulfilling their purpose in prototyping for early assessment of circuit design. This work also presents novel multicellular designs as an application of the tool for implementation and analysis of multicellular synthetic circuit designs. They mainly refer to spatial and/or temporal patterns, and make full use of the newly included features of gro. Future developments on the simulator include automating simulation specification, offering a web-based version of the simulator, connecting to existing standards 
such as SBOL or SBML and adding onto the existing features by implementing bacteriophage infection or gene editing capabilities such as a CRISPR/Cas9 system. gro has become a tool that is useful for a broader audience than its previous version. Systems biology, evolutionary biology and epidemiology are examples of other fields that could benefit from a new platform like the one presented in the current document. 


\section{Resumen}

La biología sintética es una disciplina nacida recientemente que se origina en el año 2000 en el Laboratorio de Inteligencia Artificial del MIT. Emerge como una disciplina en que la ingeniería se aplica a componentes y sistemas biológicos. Por su naturaleza, la biología sintética es un campo interdisciplinario en el que los ingenieros y biólogos trabajan en conjunto para diseñar e implementar circuitos que ya sea persiguen recrear vida, o bien comportarse de manera no natural con algún propósito específico. Hasta recientemente, ésta ingeniería de la biología se ha llevado a cabo de manera informal. No existían herramientas bioCAD o de simulación para asistir en el diseño de circuitos biológicos. Un ciclo de prueba y error en conjunto con la intuición del biólogo eran las directrices en un proceso largo con el objetivo de alcanzar el comportamiento deseado del circuito biológico. Esto coloca a la biología sintética en un estado comparable al de la microelectrónica en los años 80. El método de prueba y error es lento, por lo que simuladores y herramientas de bioCAD son requerimientos para acelerar el proceso. El ciclo de diseño-construcción-prueba-aprendizaje es un enfoque que muchos biólogos sintéticos actualmente utilizan para llevar a cabo dicha tarea. Este ciclo aprovecha plenamente el uso de las herramientas de software para acelerar el proceso. En la fase de diseño del ciclo, los científicos trabajan en un diseño y profundizan sobre su funcionalidad de manera teórica. Es en este punto que las simulaciones computacionales muestran su utilidad, puesto que reproducen las dinámicas base de dichos diseños. Estos programas tienen por finalidad ayudar en la identificación y descarte de diseños incorrectos. También sirven para dirigir y estimar valores de parámetros que luego serán utilizados en la implementación de los diseños en el laboratorio de microbiología. El objetivo es minimizar costos en tiempo y dinero que suscita la experimentación en el laboratorio. En este contexto, hay una necesidad clara de simuladores veloces, precisos y simples de usar.

Los modelos basados en agentes (AbMs), también conocidos como modelos basados en individuos (IbMs), son un tipo de modelos implementados por simuladores que se centran en el comportamiento de cada individuo en una colonia (en el contexto actual, son colonias de células). Estos modelos son alternativas a aquellos basados en Ecuaciones Diferenciales Ordinarias (ODE) o estocásticos (Gillespie). Los AbM proporcionan una mejor representación de cómo interactúan poblaciones bacterianas y se comportan de manera espacial, mientras modelos basados en ODEs y Gillespie ofrecen una simulación más precisa de dinámicas a nivel intracelular. Las colonias bacterianas crecen en 2D sobre placas de Petri. Cada bacteria se puede ver como un agente individual que se comunica e interactúa con otras bacterias a través de señales químicas del en- 
torno o por intercambio de mensajes genéticos (plásmidos). Cada célula sigue una serie de reglas probabilísticas que dirigen su crecimiento, reproducción, comunicación, muerte, o cualquier acción que ejecute. Esto representa el comportamiento completo de la bacteria. Las reglas probabilísticas implican la emergencia de un comportamiento global a nivel poblacional. Los simuladores AbM son adecuados para evaluar y predecir comportamientos de circuitos sintéticos multicelulares.

Lo expuesto conduce a hacerse las siguientes tres preguntas de investigación al respecto: 1) ¿Qué potencial tienen los simuladores AbM actuales para simular circuitos genéticos multicelulares novedosos que utilicen conjugación bacteriana? 2) ¿Cómo se pueden solucionar las limitaciones identificadas para que se puedan simular circuitos genéticos multicelulares novedosos que utilicen conjugación bacteriana? 3) ¿Qué diseños novedosos relacionados con patrones espaciales y/o temporales puede prototipar la plataforma mejorada?

Al empezar el desarollo del presente trabajo, no habían simuladores AbM capaces de simular circuitos multicelulares del proyecto PLASWIRES. gro se seleccionó como AbM para ser mejorado y extendido. A raíz de esta elección y del aumento gradual en complejidad de las simulaciones, se estableció un listado de características para complementar el simulador:

- Mejorar la velocidad del simulador: se logró implementando CellEngine, un nuevo motor físico que consta de un algoritmo de empuje adaptado para colonias de bacterias.

- Agregar conjugación bacteriana como mecanismo de comunicación intercelular: se implementó directamente en el código fuente de gro, siendo un requerimiento clave para simular circuitos de PLASWIRES.

- Simplificar el proceso de expresión genética en el simulador y la especificación de simulaciones: CellPro es un nuevo módulo que simplifica la expresión genética por medio de dinámicas asociadas a proteínas binarias. ProSpec define un nuevo lenguaje de especificación escalable para describir circuitos genéticos complejos.

- Agregar consumo de nutrientes y modulación de crecimiento celular: un simulador de consumo de nutrientes basado en Monod, CellNutrient, se desarrolló para acoplarse a gro.

- Mejorar las capacidades de señales del entorno: CellSignals empaqueta una nueva versión extendida de simulación de señales del entorno para gro.

gro se mejoró sustancialmente con la inclusión de las características propuestas. Ahora es capaz de simular $10^{5}$ bacterias en minutos (en vez de una semana). La aceleración adquirida del simulador lo ubica como una herramienta de prototipado muy rápida que se aplica a una amplia gama de diseños en biología sintética y de sistemas. Además, gro ahora dispone de especificación y dinámicas genéticas incorporadas en el sistema, consumo de nutrientes, capacidades de señales del entorno mejoradas y conjugación bacteriana. La plataforma junto con los módulos mencionados son de código abierto y se pueden encontrar 
en: https://github.com/liaupm/GRO-LIA. El realismo de las simulaciones producidas por la plataforma ha aumentado gracias a la gran cantidad de bacterias que es posible simular ahora y que ellas pueden hacer uso de las nuevas características. Los resultados de estas simulaciones muestran el comportamiento de circuitos multicelulares, cumpliendo así su propósito en el prototipado para evaluar tempranamente el diseño de los circuitos. Este trabajo también presenta diseños multicelulares novedosos como aplicación de la herramienta para implementación y análisis de diseños de circuitos sintéticos multicelulares. Estos diseños se refieren principalmente a patrones espaciales y/o temporales, y hacen uso de todas las características nuevas incluidas en gro. El desarrollo futuro del simulador incluye la automatización de la especificación de simulaciones, una versión web del simulador, la conexión con estándares existentes como SBOL o SBML y la implementación de nuevas características como infección de bacteriófagos o capacidad de edición genética como en el caso del sistema CRISPR/Cas9. gro se ha convertido en una herramienta que le es útil a un público más amplio que en su versión anterior. Biología de sistemas, biología evolutiva y epidemiología son ejemplos de otras disciplinas que se podrían beneficiar de una nueva plataforma como la que se presenta en el documento actual. 


\section{Chapter 1}

\section{Introduction}

Systems and synthetic biology are fields relating to the design and analysis of biological systems. In the case of systems biology, these constructs seek to be explained at a system level. It provides a higher level perspective upon which biological systems can be studied. Focus of study in this field is aimed towards four main goals: 1) understanding system structre, 2) studying the system dynamics, 3) controlling biological systems, and 4) obtaining methods for designing and altering systems to obtain a desired behavior or property $\mathrm{K}^{+} 01$. Building on this fourth direction, a different approach can be taken: applying engineering to biological systems. This refers to synthetic biology. Two directions arise: 1) reproducing emergent behaviors from natural biology, and 2) assemble systems that display an artificial and unnatural behavior [BS05.

Scientists working on systems and synthetic biology undergo a very long and tedious process to reach designs that can successfully be implemented in the lab. Recently, the development process for systems and synthetic biology experiments has started following the Design-Build-Test-Learn cycle [LBZG15]. Within this cycle, the design phase is the most creative and conceptual one. Ideas are put into place and represented with a model or system that should later be built and tested.

Usually, scientists hardly get any preliminary feedback on their proposed designs, as building and testing phases produce their first results Gor16. However, it is at the design phase that information about the constructs can help accelerate the development process. Basic design flaws and mistakes in the construction can be detected early if information on the execution were available. It is in this spirit that simulation software seeks to test the fundamental rules imposed by the conceived designs and serve as an early indicator of potential problems.

Designs can be simulated from different standpoints and with distinct purposes. For instance, tools such as D-VASim [BM17] are oriented towards the simulation and analysis of genetic circuit models described in SBML (Systems Biology Markup Language) $\mathrm{HFS}^{+} 03$. It focuses on a very detailed representation of genetic circuits, and from a single-cell behavioral perspective. Another system, Cello $\mathrm{NDS}^{+} 16$, is tasked with finding the correct configuration for im- 
plementing genetic circuits. It is based on Electronic Design Automation (EDA) and the Verilog language (for specifying electronic circuits) to describe hardware circuit function and hence, program cells. The resulting circuits are based on RNA Polymerase input and output signals while circuitry logic is implemented through a cascade of NOT and NOR gates. Cello seeks to reproduce the path electronics followed within the context of synthetic biology. Both D-VASim and Cello simulators are mainly aimed towards analysis in the construction of designs.

Other simulators are meant to target the study of specific processes such as biofilm formation, cell growth or intercell communication. The reader can remark that these processes involve cell populations, and no longer focus on the behavior of a single cell. Multicellularity is a next step in circuit scaling Amo14 and also crucial for providing new tools for multicellular systems biology. An example is tissue and microbiome engineering and study. Designs involving populations require observing emergent behavior, instead of individual entities. Characterization, studying and iteration at the design phase in these types of circuits also proves to be very useful Gor16.

Programming biology can therefore be interpreted in the sense of altering native components in biological hardware to change the behavior to something that is expected by the programmer. However, it can also be understood as the simulation of biological situations in which the biology behavior is programmed JOEK12, $\mathrm{NDS}^{+} 16$. Two key components are present in this context; the biological hardware and designs that use it to exhibit novel artificial behavior. The first component refers to all of the biological systems involved in wet-lab experiments. The second one relates to the interaction of these elements and how they connect to produce the expected behavior.

This interplay of elements should be tested early in the development cycle to reduce costs related to the construction of such systems in the wet-lab and to time required for analyzing the results of those experiments. This is referred to as prototyping. One of the goals for simulators is to fulfill the role of platforms to prototype designs. As stated above, a good simulation platform should execute fast, offer an integral view for anaylizing the designs being tested, should be able to simulate realistic population sizes and provide a large toolkit for describing complex circuits. Of course, the specification of such designs should also be simple enough for the scientist to easily prototype his/her designs GGGPdP $^{+} 17$.

These requirements provide guidelines for developing new features and directing improvement of existing ones. It is at the core of this problem that the current work seeks to contribute. Progress in speed and capacities of an existing platform to comply with requirements that cannot be satisfied by existing simulators is the main goal: A fast platform that can prototype synthetic circuit designs involving cell-cell communication is the desired outcome. This platform offering new features should be able to prototype novel circuits or somehow make the prototyping of exisiting and novel designs easier. Therefore, exploiting the newly developed tool is also a problem to solve. Identifying and creating designs that were not possible to simulate or were very difficult to under a better environment is another important goal. 
The core of this work shows the improvement of an AbM simulator, gro, in acquiring new capabilities and making it more powerful. gro is supplied with a new physics engine that now is able to handle about 2 orders of magnitude more simulated cells than the original version in a lower time. Also, capabilities concerning cell-cell communication (bacterial conjugation and environmental signals), cell growth and nutrient consumption, gene expression dynamics and circuit specification are presented. All of these features were either implemented from scratch and adapted to the simulator or are the result of revising existing features that were extended and improved. Also, new synthetic designs are proposed and simulated in the newly created platform. These simulations take advantage of the novel features of the simulator and therefore also present examples of how the extended toolkit is used to advance study of synthetic biology through the presented version of gro.

This document is structured as follows: Chapter 1 (this Introduction) establishes the angles from which the research questions were posed. It introduces the context upon which the system was developed and the directions for which the development constitutes an advance. Chapter 2 reviews basic concepts on systems and synthetic biology, well-known circuit designs and other existing simulators. Chapter 3 states the research questions and goals that were pursued in this work. Chapter 4 describes the main contribution of this work, first by presenting the improvements and novel additions made to gro and then by presenting novel circuit designs simulated on the platform. Chapter 5 concludes and proposes future research and development lines. Appendix $B$ is a compendium of all the source codes of simulations used in this document. Appendix $\mathrm{A}$ is a reference manual for all of the new additions that were made to gro. 


\section{Chapter 2}

\section{State of the art}

\subsection{Synthetic biology}

Synthetic biology is an interdisciplinary young field in which engineered devices are constructed using biological hardware: in this context, hardware refers to the elements involved in microbial function. Examples include DNA (deoxyribonucleic acid) and RNA (ribonucleic acid) strands, proteins, intercellular signals or ribosomes among others. Interaction between these "pieces" give rise to specific behaviors. In nature, these elements are pre-organized to follow a given behavior. However, what would happen if the pieces were to be rearranged according to an specific design? Could this new arrangement exhibit a pre-concieved but unrelated behavior? Could it be viewed as a programmed "machine"?

A definite answer to these questions was revealed in the year 2000, when two foundational "machines" (or circuits) were published: A cyclic genetic oscillator in which three genes express themselves in a given order [EL00] and a genetic toggle switch implemented by two genes that are mutually repressing each other GCC00. These devices (executed inside microbial cells) behaved in an artificial and programmed manner, marking the start of the field.

Following these circuits, more toggle switches ASMN03 and oscillators $\mathrm{FWS}^{+} 05$, $\mathrm{SCB}^{+} 08$, TMLSF09] were developed, and the field expanded into other areas such as digital logic gates and information processing $\left[\mathrm{WB} 02, \mathrm{WBH}^{+} 03\right.$, HMC02, AVA07, pattern generation $\mathrm{BGC}^{+} 05$, $\mathrm{SHP}^{+} 09$, implementation of bacterial ecosystems $\mathrm{BSO}^{+} 08$, pathway engineering $\left[\mathrm{MPW}^{+} 03, \mathrm{RPO}^{+} 06\right]$ and use of negative feedback for engineering stability in gene networks BS00. Many of the engineered devices are focused on the use of artificial gene regulatory networks (GRNs) and the processes that accompany them. Examples are DNA replication, transcription and translation, induction and repression of regulation by external inducer molecules, RNA operations or gene editing among many others. A good review of these applications was published by Khalil and Collins $\mathrm{KC10}$.

In this initial stage of synthetic biology, devices were implemented as modules (the Repressilator and Toggle Switch circuits are examples) where only 
genes and their interactions were the building blocks for devices following a bottom-up approach. This is known as the first wave of synthetic biology. A few years later, circuits started to be built by putting together or manipulating systems. The definition of a system is that of a module aggregation that interacts and exhibits a reproducible and coherent behavior Amo14, PW09. This second wave of synthetic biology persists until today, as the construction of circuits is still largely based on systems. A third wave is underway: Amos indicates in his paper Amo14 that the next step in constructing devices in synthetic biology will rely on networks. Synthetic biology is reaching a stage in its maturity where standards are becoming a necessity [End05, Ark08] in that the design and implementation of synthetic devices are a complex task and require full characterization of its components.

The field is therefore an application of engineering concepts to biology $\mathrm{KKA}^{+} 04$, ABKW06 in which the scientists either aim to recreate life or use biological modules for constructing systems with a specific purpose [BS05]. In the spirit of understanding the involved elements and their role, it is fundamental to review the basic concepts associated with the construction of synthetic biology devices.

\subsubsection{Gene Expression}

Cellular function is based on DNA code. The information stored in DNA sequences is composed of nucleotides which form the sequence alphabet and constitute the basic information unit. There are four types of nucleotides (also called bases): A (adenine), C (cytosine), G (guanine) and T (thymine). DNA sequences can be single-strand or double-strand. Following the Watson-Crick complementarity principle [CW54, A pairs with $\mathrm{T}$ and $\mathrm{C}$ pairs with G. RNA also possesses this structure for information storing (however, in the case of RNA, T is replaced with $\mathrm{U}$ - uracil), and also presents the complementary nature of nucleotides.

DNA undergoes a process in which it is transcribed into RNA which is then translated into a protein. These operations are called transcription and translation respectively. In this document, focus will be set on prokaryotic organisms, since it will be the type of entities that will be used (specifically, a bacterium called Escherichia Coli, which is of type Gram-negative). This is also of key importance, as transcription produces readily translatable mRNA (messenger RNA) molecules. In contrast, eukaryote organisms display a more complex process involving several stages before reaching translation.

Transcription occurs by copying a DNA base into its RNA corresponding base - all of them transcribe into the same base, except $\mathrm{T}$ that transcribes into a U. This process is carried out by an enzyme called RNA polymerase. It reads DNA and using it as a template, produces output mRNA. Once transcription has finalized and the resulting mRNA has been produced, ribosomes read the mRNA molecule and initiate the translation process. At this stage, ribosomes take triplets of mRNA bases, called codons, and couple them with a tRNA (transfer RNA) codon complementary (called anticodon) to the mRNA. These anticodons being used by the ribosome are bound to an amino acid. There- 
fore, each codon is associated with a specific amino acid through the action of enzymes called Aminoacyl tRNA synthases. These enzymes are in charge of pairing up the codons in mRNA with the appropriate amino acid. accounting for the translation. 20 different amino acids exist and map to the 64 possible codons. Upon binding the mRNA codon with the tRNA anticodon, the amino acid is appended to a chain that forms a protein. In sum, proteins are amino acid sequences. Once the protein has been produced, its structre folds into a three-dimensional structure which is key in determining its function, and for carrying it out properly.

Genes are DNA regions that encode for functional proteins in a cell. Therefore, the process that has been described for producing proteins inducing a certain function in the cell is known as gene expression and is an important process for the construction of synthetic systems. Gene expression constitutes the most basic form of inducing function, starting from a DNA template, within a cell. The process is depicted in Figure 2.1. 


\section{DNA sequence (coding strand) \\ mRNA sequence \\ DNA sequence \\ (template strand)}

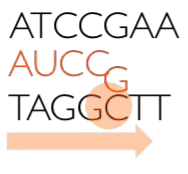

Transcription

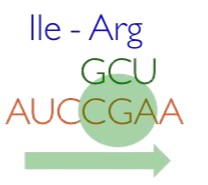

protein (amino acid chain)

tRNA anticodon

mRNA codon

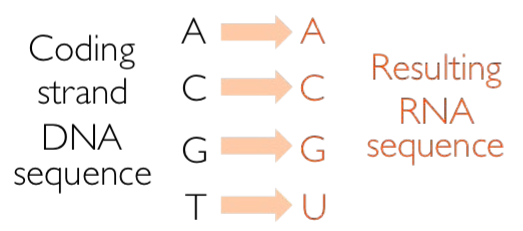

Translation

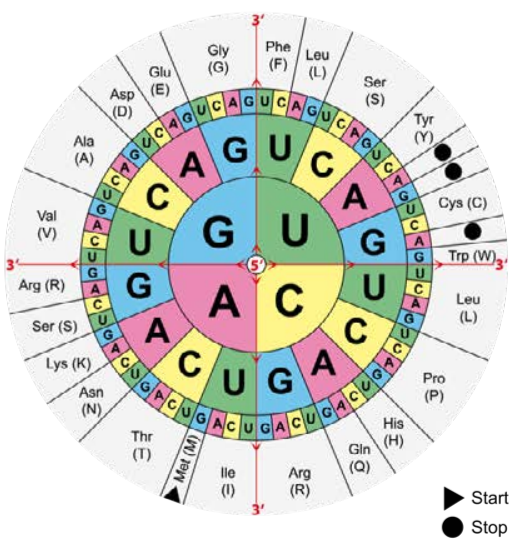

Figure 2.1: Gene expression process. In prokaryote organisms, gene expression occurs in two steps: transcription and then translation. The orange circle represents RNA polymerase, the responsible enzyme for transcribing DNA into mRNA. It elongates RNA by hybridizing with the template strand of the DNA. Transcription occurs following the rules stated in the lower left part of the figure. A green circle in the top right represents the ribosome. It translates an mRNA codon into an amino acid, which is a unit in the protein chain being formed. Translation occurs following the information shown in the lower right, the codon wheel. This chart can be read by following the nucleotides outwards. The inner circle represents the first nucleotide, the second one is read in the adjacent circle, and the third one is in the outer circle. Outmost names represent the amino acid to which the codon is paired.

\subsubsection{Gene Regulatory Networks}

Proteins convey function in a cell. One of the protein functions is to induce or prevent another (or the same) gene or group of genes from producing proteins. Transcription factor $(\mathrm{TF})$ is the name given to a protein controlling gene expression. TFs can be of two types: activators, which induce gene expression or repressors, which block and prevent the process. TFs are therefore responsible for regulating gene expression.

In a DNA sequence, genes are preceded by a region called promoter. The role of promoters is to initiate transcription. The RNA polymerase binding site is contained inside the promoter. This is the zone where RNA polymerase binds to the DNA template sequence and starts to transcribe. Another region that 
influences gene expression regulation is located downstream of the promoter and is called operator. Operators are DNA regions where a repressor binds and blocks transcription, in turn preventing gene expression. A promoter may be regulated by zero or more TFs. Promoters that do not require any TF to initiate transcription of mRNA are classified as constitutive.

The DNA sequence formed by a promoter, an operator and all of the genes regulated by the same promoter is called operon (shown in Figure 2.2p). Therefore TFs have an effect on all genes of the operon in that either all or none of them are expressed.

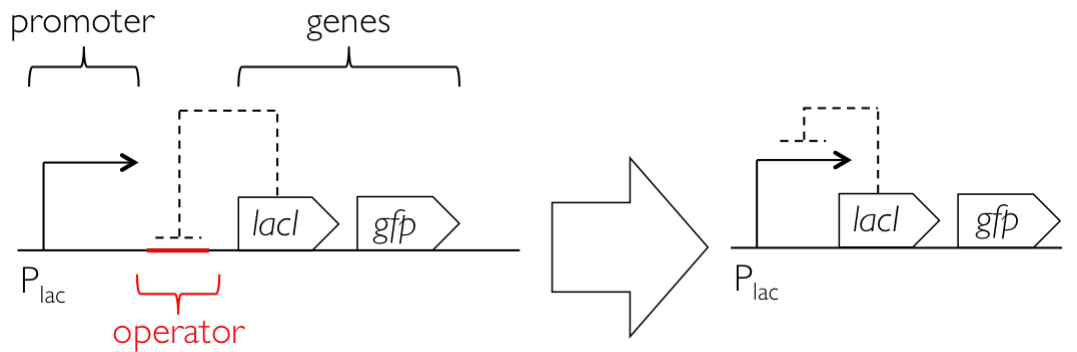

Figure 2.2: Depiction of an operon. A promoter region called $P_{l a c}$ is in charge of initiating mRNA transcription. Genes lacI and GFP are under the control of $P_{\text {lac }}$. lacI gene produces a TF that represses transcription by binding to the operator region of the operon. Standard notation for the remainder of this document is shown here: promoters are represented as bent arrows, the bluntend arrow signals inhibition or repression (conversely, normal arrows stand for activation), the pointy end rectangles are genes and generally the operator is omitted, as shown in the simplified depiction on the right. A complete reference for symbols was published by Quinn et al. as SBOL Visual QCIA $^{+} 15$.

Each of these interactions can be arranged in cascade, making an operon produce a TF that regulates the expresion of itself or another operon [SBB00, forming a network of interconnected operons, Gene Regulatory Networks (GRNs). GRNs give rise to complex interactions and implement the building blocks of life. These networks stand at the base for design and implementation of synthetic circuits, as the interactions can be repurposed for specific artificial functions.

Furthermore, operons are part of larger structures called plasmids. These are circular fragments of double stranded DNA that contain mobile data and usually confer some advantageous trait to the host $\left[\mathrm{SBH}^{+} 05\right]$. Plasmids bear a copy number, being the maximum amount of plasmid copies that are found in a bacterium. Finally, the main DNA fragment of a host is the genome. It is also a circular piece of double stranded DNA and accounts for the largest amount of DNA in the host. 


\subsubsection{Network motifs}

GRNs are organized in the most diverse topologies and complexities. However, patterns of network organization are recurrent across several types of organisms. This suggests that specific functions are performed by each pattern independent of their nature. These patterns are called network motifs $\mathrm{MSOI}^{+}$02, SOMMA02 and constitute basic building blocks for larger GRNs Alo07.

The best known motifs identified and studied by Alon involved in GRNs are Feed-forward Loops (FFLs). FFLs are described as three node structures in which there is a double interaction between an "entry" node and an "output" node. These interactions coexist and differ in that one of them is through an "intermediate" node and the other one is direct. Each of the interactions may be an activation or a repression. Also, since the "output" node is on the receiving end of the interactions, the input signals may be processed as an AND gate or as an OR gate. Eight types of FFLs have been identified and classified into two groups according to the disposition of their interactions: Coherent FFLs and Incoherent FFLs MA03]. Figure 2.3 shows the classification of FFLs.

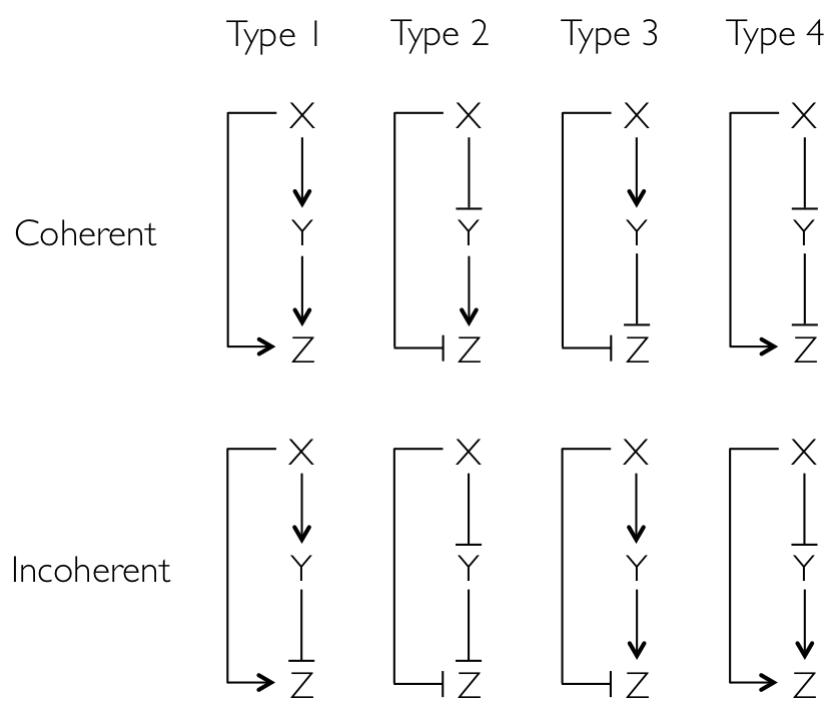

Figure 2.3: Feed-forward Loops. Eight types of FFLs exist, being classified into Coherent (CFFL) and Incoherent (IFFL). For all FFLs shown, the "input" node is called X, the "intermediate" node is called Y and the "output" node is Z. CFFLs display a coherent output between both the direct and indirect arms of the structure, this is both arms either result in an activation or in a repression. IFFLs are the opposite, both arms have a different resulting interaction: one of them is a repression and the other one an activation. Figure adapted from Mangan and Alon MA03.

In their paper, Mangan and Alon also provide a summary for the function of FFLs. An adapted version of their information is shown in Table 2.1. 


\begin{tabular}{ll}
\hline Function & FFL class and type \\
\hline Steady state logic sensitive to X and Y values & AND CFFLs types 1 and 2 \\
& OR IFFLs types 3 and 4 \\
Delay upon X value change & CFFLs types 1, 2, 3 and 4 \\
Pulse generator gated through Y and dependent & OR IFFL types 1 and 2 \\
on X value & AND IFFL types 3 and 4 \\
& (with no basal Y value) \\
Acceleration upon X value change & IFFL types 1, 2, 3 and 4 \\
Cooperativity enhancement on X value & (with basal Y value) \\
& AND CFFL type 1 \\
\hline
\end{tabular}

Table 2.1: FFL functions. A group of generic functions dependent on the input and changes for $\mathrm{X}$ and $\mathrm{Y}$ values was compiled in MA03. $\mathrm{X}$ and $\mathrm{Y}$ values can be assigned a Boolean value representing their expression. The function is expressed as an output in the $\mathrm{Z}$ node.

In sum, these recurring constructs along with custom interactions serve as the building blocks to engineer genetic circuits. Schaerli et al. $\mathrm{SMG}^{+} 14$ demonstrate applications of IFFLs to pattern generation (specifically stripes).

\subsubsection{Intercellular communication}

Microbial organisms have been proven to communicate through many different channels under varied circumstances. Some of these mechanisms involve the environment (long range), while others occur with cells in proximity (local range). The messages that are transferred between cells also vary in their form with the different mechanisms. In this section, two of them will be reviewed: Quorum Sensing and Bacterial Conjugation.

\section{Quorum Sensing}

One of the best studied intercellular communication mechanisms is Quorum Sensing (QS). It is a signaling system that was first identified in the luminescence system (Lux operon) of Vibrio Fischeri bacteria [NPH70]. The signaling system produces small molecules called autoinducers [NH79]. Two components form QS systems: an emitter and a receiver. Emitters produce autoinducers, specifically in the case of Gram-negative bacteria $\mathrm{WBS}^{+} 01$, N-acyl homoserine lactones (AHL). These AHL molecules are then released into the environment and sensed by receivers. The Lux system is depicted in Figure 2.4 . 


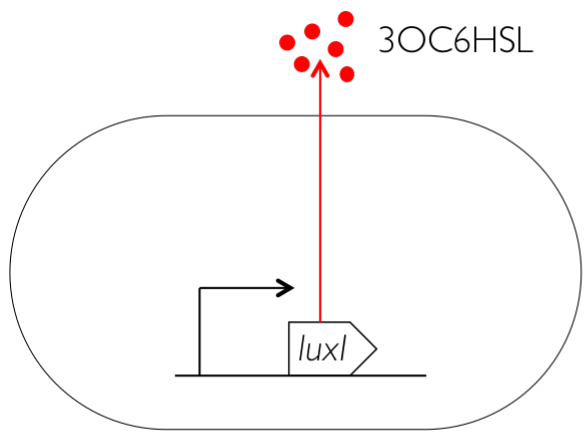

Emitter cell

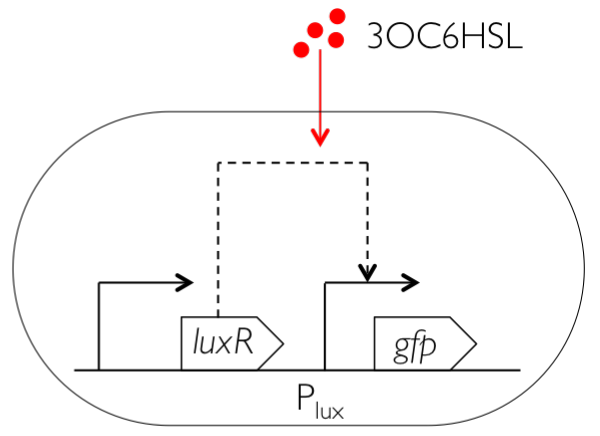

Receiver cell

Figure 2.4: Lux QS system. The emitter cell produces AHL autoinducer (3-oxoC6-HSL, abbreviated to 3OC6HSL) molecules when protein luxI is expressed, and then releases those molecules into the environment. AHL is later sensed by the receiver cell form the environment in a process where protein lux $R$ binds to the AHL molecules and serve as a TF for promoter $P_{l u x}$.

The dynamics can be summarized as follows: when autoinducers are detected in high concentration, the operon induces their further production, although, when auto-inducers are sensed in low concentration, a lower output of them are produced. Therefore, QS is a mechanism in which cells can estimate the population density FWG94.

QS is considered a long range communication mechanism, since AHL molecules are released into the environment and can be sensed by distant cells. QS systems have been deeply studied MB01, WB05, PB16. Apart from the Lux system, other QS systems have been found [MB01]. An important property that must hold between QS systems is orthogonality, which refers to the lack of interference among systems. This is, molecules emitted by one system should not be recognized by another one. Conversely, crosstalk is the term used for situations in which systems interfere. Scott and Hasty published a recent paper in which they assess the levels of crosstalk between QS systems and study different types of orthogonality for their use in synthetic circuits [SH16].

Using the features of QS, programmability is harnessed from these systems. This is achieved by linking the receiving system to some downstream functionality (as shown in Figure 2.4). Collective behaviors are attained through this communication system, as AHL molecules can be treated as signaling components. Because of this, many synthetic circuits use QS as the communication backbone between bacteria. Notable examples include a circuit for programmed cell death [YCWA04, an edge-detecting circuit [TSS ${ }^{+}$09] and a band-pass filter circuit $\mathrm{BGC}^{+} 05$.

\section{Bacterial conjugation}

Despite being a general communication system, QS cannot be spatially directed. Also, the amount of information exchanged through QS is poor OE12, 
GMAdlC13. Since AHL molecules do not carry genetic information and are only perceived through their density, message transfer is slow and does not carry much information. To increase message throughput, a larger amount of data should be exchanged.

DNA provides a viable system for storing a substantial amount of data and transferring it CGK12, $\mathrm{YNFR}^{+} 14$, EZ17. Transfer of DNA can occur in two ways: vertically or vertical gene transfer - VGT (DNA in a daughter cell is inherited from the mother) or horizontally, also known as horizontal gene transfer - HGT [DlCD00] (no direct inheritance occurs and DNA is transferred to an unrelated cell).

Several HGT mechanisms are known in prokaryotes, two important ones being: bacterial conjugation [TL47, LGRCC02 and bacteriophage infection $\mathrm{OE} 12, \mathrm{CFCC}^{+} 03$. Bacterial conjugation is a local transfer mechanism in which a host holding a conjugative plasmid (capable of being transferred on its own), transfers it to an adjacent bacterium. Bacteriophage infection refers to the propagation of DNA through viruses that infect cells within a certain range of the source. This section will maintain the focus on bacterial conjugation.

Bacterial conjugation is a complex process through which genetic material is transferred from a donor host to a recipient. The mechanism is mediated by several proteins and key sites in the plasmid that establish the transfer channel:

- An oriT site: a non-coding DNA region in a plasmid that marks where one of the strands is nicked to initiate transfer of the plasmid.

- Relaxase: it is a protein that nicks one strand of the plasmid and starts its transfer.

- T4CP: protein that directs the relaxase bound to the transferring plasmid towards the transport channel.

- T4SS: protein-based system that produces the secretion transport channel through which the relaxase and plasmid are transferred.

This process is thoroughly described in a paper published by Smillie et al. [SGB ${ }^{+}$10], also, it classifies plasmids in mobilizable (can be transferred, but is lacking certain elements to be completely capable of transfer) and conjugative. Conjugation is shown from a general perspective in Figure 2.5. 
a)

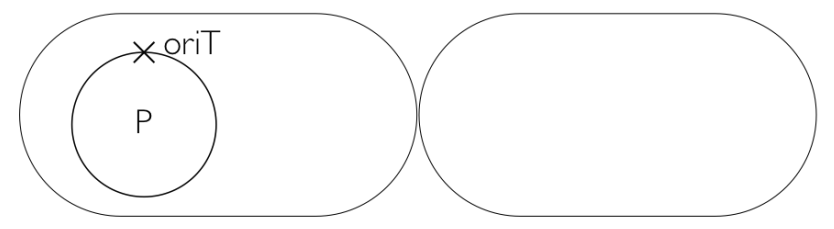

b)

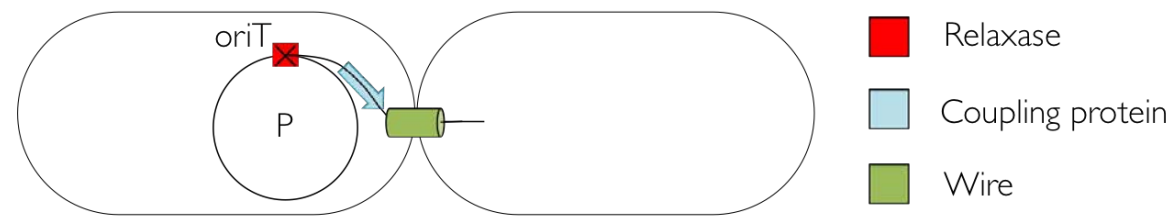

c)

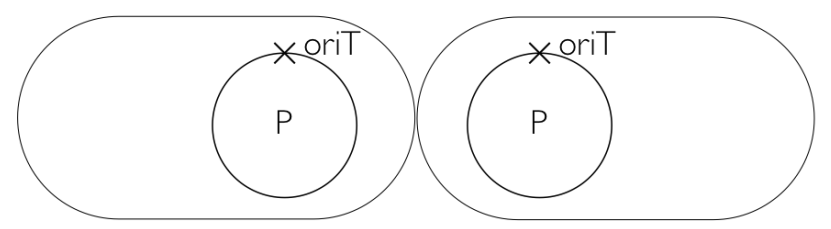

Figure 2.5: Schematization of conjugation. Several stages are involved in bacterial conjugation: a) A conjugative plasmid $P$, that is carrying all of the conjugation machinery (Relaxase, Relaxase Accessory Proteins (RAP): coupling protein and wire) is present in a bacterium but not in its neighbor. b) The relaxase nicks one of the strands of $P$ and the coupling protein leads the relaxase along with the plasmid strand towards the T4SS channel (wire). c) DNA repair mechanisms act on each of the single stranded plasmids on both bacteria and rebuild the missing part of $P$ for it to be double stranded again.

However, bacteria have a mechanism for blocking the access of plasmids that share a compatibility group GBdlC08. Therefore, with all of the features described here, programmability of conjugation is possible by handling the involved components. Being an important transfer mechanism, conjugation will be used heavily throughout this work.

\subsection{Multicellular circuits}

Programming single cells requires coupling genetic device pieces together for them to execute a specific function. Each of these modules or systems impose a metabolic burden on the cells. This yields a limitation on what a single cell can perform on its own. Therefore, an alternative approach is to distribute function among many cells [MPS12], similar to how a computer network operates GMAdlC13.

This philosophy requires identifying all components for carrying out the task at hand. Also, a robust communication system is needed for the entities to share information concerning the division of labor and results of intermediate processing. Early examples of these circuits include a pattern generation circuit 
BGC ${ }^{+}$05] and genetic logic gates GMAdlC13, TTV11, $\mathrm{RMC}^{+} 11, \mathrm{MMC}^{+} 16$.

Under such a paradigm, scalable circuits can be constructed, and larger outputs are achieved. The use of multiple populations, or microbial consortia, opens the door for engineering comples tasks BYA08. The correct functioning of the circuits relies on the communication backbone. For bacteria, three approaches have been proposed: QS $\left[\mathrm{BGC}^{+} 05\right.$, TTV11, bacteriophage infection OE12 and conjugation GMAdlC13. This work uses two communication systems for the implementation of presented circuits; QS and conjugation.

\subsection{Temporal and spatio-temporal pattern cir- cuits}

Patterns typically refer to recognizable and regular arrangements or sequences. Many types of patterns exist, however, the focus of this section will be on microbial populations exhibiting temporal and spatio-temporal patterns. A temporal pattern is usually associated with a sequence that is either repeated or exhibits a distinctive and predictable behavior in time. A spatio-temporal pattern adds a spatial component that is observable and recognizable over time.

Spatio-temporal patterns were one of the early results obtained by synthetic circuits. Achieving such patterns requires processing of cell populations, since any pattern is visible in time or space contexts. Therefore, the implementation of circuits to display patterns should keep in mind the engineering of whole populations.

Characterization of patterns resulting from models such as the Turing model or those involving morphogens KM10, or network interactions $\mathrm{SMG}^{+14}$ have been described and serve as a basis for designing new circuits displaying patterns. Interactions between microbes can be organized according to these models and drive the construction of multicellular circuits for this purpose.

On the other hand, temporal patterns do not necessarily have a spatial component, meaning they are not always visible in the form of a shape, location or visual representation. However, a sequence pattern can be recognized over time in measuring other variables, such as a sound sequence PE85] or oscillations KM10, RSLY12, KK17.

Some notable examples of spatio-temporal patterns achieved through synthetic circuits will be briefly described in this section.

\subsubsection{Bullseye pattern}

A bullseye pattern was first obtained through a genetic circuit in a foundational work published by Basu et al $\mathrm{BGC}^{+} 05$. In this paper, the authors use sensibility in a lac promoter as a way for directing the circuit response. Specifically, the level of received AHL modulates the activation of alternative repression paths. This leads to a circuit that reports a mid-level AHL concentration by expressing 
GFP. Figure 2.6 summarizes the design presented in the paper and shows what expected pattern should be produced by the circuit.
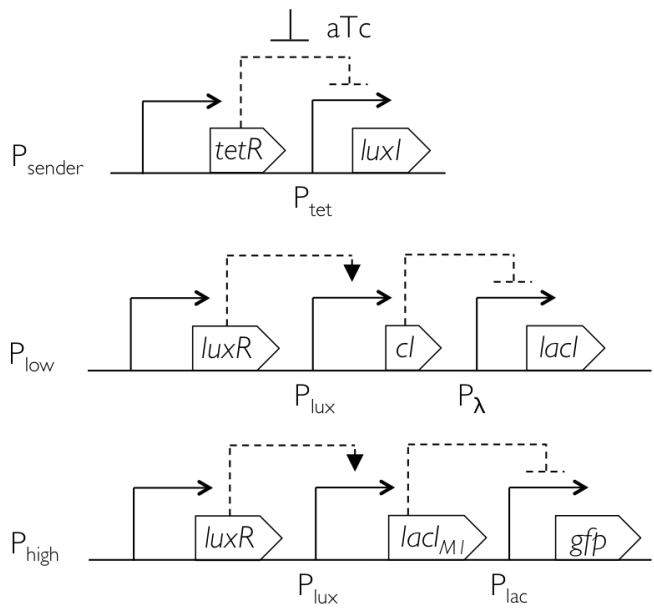

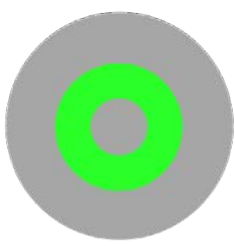

Expected pattern

Figure 2.6: Original bullseye pattern by Basu et al $\left.\mathrm{BGC}^{+} 05\right]$. The circuit differentiates two roles: sending and receiving AHL. Sender bacteria are located at the center of the plate area. Receiver bacteria grow all over the plate and contain plasmids $P_{\text {low }}$ and $P_{\text {high }}$, that respectively detect a low and high concentration of AHL. Both plasmids repress the expression of GFP through a specific type of lacI protein: $P_{\text {low }}$ expresses the regular version of $l a c I$, while $P_{\text {high }}$ generates $l a c I_{M 1}$ (a codon-modified version of $l a c I$ ). If any of the $l a c I$ variants are activated, they repress the expression of $G F P$. Therefore, the circuit should report a band in which a mid-concentration of AHL is read by expressing GFP. On the right, a depiction of the pattern is shown, where green color indicates the region that should glow, and gray represents all other regions where bacteria grow, but do not report anything.

\subsubsection{Edge detector}

Detecting the edge of a light spatial pattern with engineered bacteria was first proposed by Tabor et al [TSS $\left.{ }^{+} 09\right]$. Specifically, this work used photosensitive bacteria not detecting light to trigger the emission of AHL. Conversely, photosensitive bacteria in the light and sensing a sufficient amount of AHL, express a dark pigment. Therefore, only bacteria near the border will be marked with the pigment, delimiting the edge. Figure 2.7 summarizes the circuit design. 
a

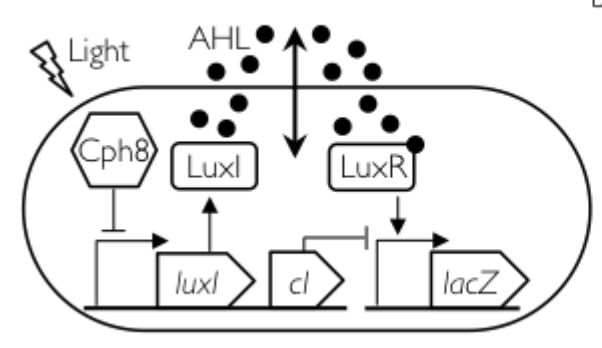

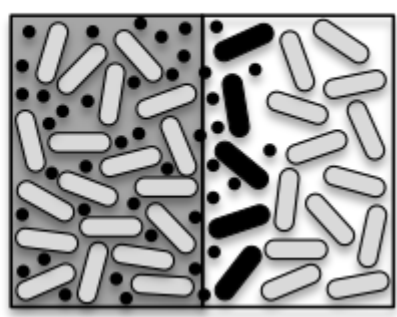

Figure 2.7: Figure inspired on edge detecting circuit by Tabor et al $\mathrm{TSS}^{+} 09$. a) Summary of the edge detecting circuit. Bacteria located in the dark side of the plate emit AHL. AHL is then detected by bacteria in the light side of the plate, which emit a pigment (lacZ) if the amount of sensed AHL is high enough. b) Only bacteria near the interface between light and dark (on the light side) express the pigment.

\subsubsection{French flag model}

Tuning bacteria to detect given concentrations of an underlying morphogen gives rise to bands being expressed. This circuit is referred to as a band-pass filter. Using this idea, Sokha et al. $\left[\mathrm{SHP}^{+} 09\right]$ constructed intersecting zones of morphogens and applied the band-pass circuit to achieve custom bacterial patterns. Figure 2.8 summarizes the model and how it is used.

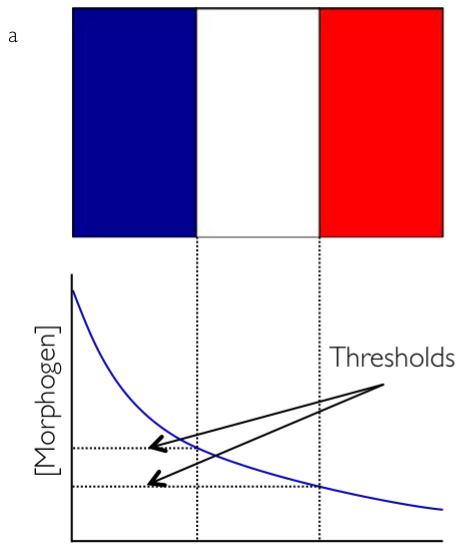

Distance to center

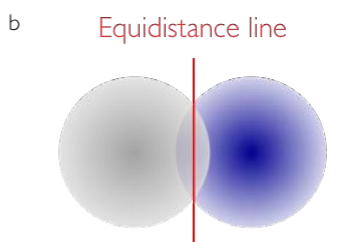

Morphogen I Morphogen 2

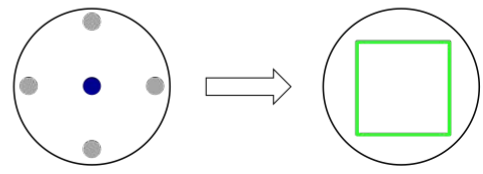

Obtained pattern

Figure 2.8: French flag model. Figure adapted from $\left[\mathrm{SHP}^{+} 09\right]$. a) Bands are generated from detecting thresholds of morphogen concentration. For a single compound and when two different thresholds guide the detection, three bands are generated. b) If two morphogens are present, an equidistant line from both sources can be used to compute custom patterns. An example is shown in which a square is constructed using a generating morphogen distribution. 


\subsection{AbM simulators}

Simulators provide a platform for prototyping and testing proposed designs. Many types of simulators exist, being the most popular ones those based on Ordinary Differential Equations (ODEs) and those based on agents (AbMs). ODE based simulators are very accurate in modeling precise dynamics at an intracellular level [KS08]. Stochasticity can also be included using the Gillespie algorithm Gil77. These elements can simulate all of the processes occurring within a cell with high precision. This accuracy and detail comes at the cost of heavy computation and the poor capacity of these models to simulate populations. AbM simulators however focus on the behavior of single entities (agents or individuals) and simulate an environment in which they interact. The models behind AbMs are generally approximations to lower the computational cost of their execution. Also, the goal is to simulate populations and explore collective concurrent behavior. In the context of this work, a multicellular approach is required, therefore $\mathrm{AbM}$ simulators are those better suited for this purpose.

The key concept behind an AbM simulator is to focus the attention on each individual entity and establish its behavior. This design paradigm is bottomup, as the resulting collective behavior should emerge from the interaction of all individual actions. Some key simulators will now be briefly described. A detailed review of $\mathrm{AbM}$ systems can be found in recent works by Hellweger et al. $\mathrm{HCC}^{+} 16$ and Gorochowski Gor16].

\subsubsection{Krone et al. Interacting Particle System Model}

An Interacting Particle System (IPS) is a continuous-time Markov jump process for describing the collective behavior of stochastically interacting components (particles). IPS represent a continuous-time equivalent to stochastic cellular automata. An Interacting Particle System (IPS) presented by Krone et al. $\mathrm{KLF}^{+} 07$ deserves special attention as it is the first tool that was presented for simulating bacterial conjugation. This simulator also engaged in elucidating the effect of nutrient diffusion and consumption have on conjugation. The system is based on a $2 \mathrm{D}$ square lattice (of size $1000 \times 1000$ ) where each position can hold several types of bacteria and nutrients. The system also allows for a second layer of cells to be present at a single lattice position. This is done to allow for a limited version of $3 \mathrm{D}$ stacking cells. Cell division is also accounted for and is done by placing a new cell at an adjacent lattice location provided that there is space left (no more than two cells at the same lattice position).

This simulator, although using discrete entities to represent cells and not modeling the full extent of cell growth, can achieve the simulation of complex scenarios involving bacterial conjugation. The whole dynamics of the system is based on neighborhood computations and eliminates the complexity of edges by establishing periodic boundaries. The model behind this simulator states rules that change the state of a lattice position. The operations that alter the state of a location are: Reproduction, segregative loss, conjugation, repression (of a plasmid), update (at a certain time step) and nutrient diffusion. 
This tool provided a great deal of insight into the underlying processes of plasmid loss and transfer. In fact, Krone et al. compare the simulation results to experimental data in their paper and reach the conclusion that their system models conjugation adequately. The simulator was designed to be a lightweight one to explore the basic mechanisms of bacterial conjugation and nutrient diffusion and consumption. It was by no means intended towards the use of conjugation as a mechanism involved in information transfer used by a synthetic circuit.

\subsubsection{BactoSim}

BactoSim [GRP15a, PGRP16] is an AbM simulator developed by Antonio García at LIA-UPM. This software is implemented atop REPAST Col03. (based on Java) and also uses discrete circular entities to represent bacteria shown in a Graphical User Interface (GUI). Parameters are set in an XML file that is processed by BactoSim. The environment in which the bacterial colony simulation takes place is a $1000 \times 1000$ grid (roughly corresponding to $1 \mathrm{~mm}^{2}$ ), where each location can hold a certain amount of nutrients and a cell. Nutrients are consumed in iterative maximum size neighborhoods, simulating diffusion (like in the model presented by Krone et al. $\mathrm{KLF}^{+} 07$ ]. The system is capable of simulating $10^{5}$ cells in 20 minutes of real time, corresponding to a simulated time of 600 minutes.

Both intracellular and intercellular processes are carried out in BactoSim: nutrient uptake and diffusion, cell division, T4SS expression, cell shoving and conjugation GRP15b. Each process is executed on a cell and in a random order, and naturally changes the bacterium state. Since the simulator is mainly concerned with studying conjugation, each bacterium is assigned a label that represents their plasmid state. These labels are: D for donor (the bacterium holds a conjugative plasmid), $\mathrm{R}$ for recipient (the bacterium does not possess the current plasmid) and, $\mathrm{T}$ for transconjugant (the bacterium did not previously have the plasmid, but received it from another transconjugant or a donor). A parameter $\gamma_{0}$ is crucial in directing the conjugation dynamics. Its value refers to the number of conjugations a bacterium performs on average during its lifetime.

The goal of BactoSim is to study the conjugation process. The main advances provided by BactoSim are: 1) it predicts conjugation behavior in bacterial populations, and 2) is useful to study how intracellular processes interact and show emergence, and affect global dynamics of conjugation. Individual behavior leading to the emergent large scale modeling of conjugation can therefore be inferred by simulating with BactoSim. The source code can be found at: https://github.com/antonio-pgarcia/haldane

\subsubsection{DiSCUS}

DiSCUS GMAdlC13, GMA15 is a framework developed to use conjugation as a tool in synthetic circuits and study the process of conjugation itself. It features rod-shaped bacteria of variable length and fixed width, and also offers microfluidic chamber settings. This simulator is implemented as a Python package in 
which every bacterium follows a given behavior programmed by the scientist, concurring with the definition of an AbM. It was the first framework to simulate conjugation with rod-shaped bacteria.

Cell growth and division are handled respectively by a parameter called textttgrowth speed and by the cell length: division occurs deterministically when the cell reaches 2 times the minimum length value. Biomechanical properties were validated against E. Coli experimental data extracted from a microfluidic channel using the same setting.

Bacterial conjugation is modeled as springs connecting neighboring bacteria and through which the DNA is transferred. Plasmid copy number is also recorded at each stage of the simulation. Conjugation occurs with a probability distirbuted according to the following parameters:

- $N$, the number of conjugation tries a bacterium has in its lifetime

- $p$, a success probability of each try

- $c_{-}$time, being the lapse of time during which a cell remains connected to another for conjugating

This simulator also has gene expression capabilities that drive individual bacterial behavior. It is represented as ODEs that are solved individually within each cell. These ODEs are (unrealistically) deterministic in solving gene expression dynamics. However, it is unclear that this approach is scalable due to the large amount of processing needed to solve each ODE, and to how complex a circuit can be simulated. The source code of the first version of DiSCUS is available at: https://code.google.com/archive/p/discus/

\subsection{4 iDynoMiCS}

iDynoMiCS $\mathrm{LMM}^{+} 11$ is the successor of BacSim KBW98, the first AbM bacterial simulator ever proposed. iDynoMiCS is a $3 \mathrm{D}$ environment for studying cell growth in a biofilm immersed in a liquid medium. The ultimate goal of the platform is to understand how individual level behavior affects an emerging population or biofilm level properties. Another important goal of the platform is to simulate spatial structure and assess its effect on growth dynamics.

Each of the bacterial entities in iDynoMiCS can be either (natively) spherical or rod-shaped bacteria to which physical shoving is applied. The rod-shape adaptation of the bacterial entities was implemented by Jean Pierre Arteaga at LIA-UPM. In addition, Aurora Arroyo, also from LIA-UPM, developed the simulation of conjugation for the platform. Another important feature of $\mathrm{iDy}-$ noMiCS is the simulation of extracellular polymeric substances (EPS) that are involved in biofilm formation, along with erosion and shear forces, and are accounted for in the microbial mechanical interactions. Non-bacterial agents may also be introduced into the system. A discrete time step is the duration for an agent to update its state. All of the agents process this update in a random order. 
Microbial behavior and metabolism are based on the sensing of local solute concentrations present in the environment, reactions and mechanical interactions between agents. Three layers are involved in the simulation process: a biofilm layer, a boundary layer and a bulk layer. All of the microbes and EPS produced by them and involved in the simulation are concentrated in the biofilm layer. On top of this layer, the boundary layer, is a diffusive liquid region between the biofilm and bulk layers. Finally, the bulk layer is composed of wellmixed bulk liquid where the whole system is immersed and solute concentrations are fixed to their bulk values.

iDynoMiCS is implemented in Java, with a configuration of its parameters being expressed in XML. This parametrization, despite being very thorough and complete is complex. The simulator has been validated against experimental biofilm data. The simulator can be found at: http://www.idynomics.org

\subsubsection{CellModeller}

CellModeller RSPH12 is a fast Python-based 3D bacterial simulation programming environment where users can implement cell behavior and visualize it in a GUI. This simulator was developed by Tim Rudge and P.J. Steiner at University of Cambridge. It can run on GPU hardware (using OpenCL) and simulate up to 30000 cells in 30 minutes. The source code of CellModeller was released in March 2015, and can be found at: https://github.com/HaseloffLab/CellModeller

The AbM framework assumes each agent to be a rod-shaped cell. These cells divide when they reach a length uniformly distributed between 3.5 and $4.5 \mu \mathrm{m}$. Each cell possesses a state given by a set of physical and biological variables. CellModeller implements a novel and sophisticated biophysics model that is well suited to be processed in parallel. When the cell divides, by default, the two daughter cells inherit the state. Intercellular environment signals are included in the CellModeller toolkit. At the time of its release, conjugation was not available in the simulator.

Cell behavior can be specified in a rule-based fashion or using ODEs. The process (at each time step) in which the simulation state is updated can be summarized as: 1) Update all cells according to the defined rules and ODEs. 2) Divide all cells scheduled to do so at the current time step. 3) Calculate and apply growth of a time step to all cells and reposition the cells accordingly. 4) Calculate and apply update of a time step to all extra-cellular and environmental elements. 5) Update state variables for all cells and repeat from step 1). This cycle is processed in an efficient way using GPU and CPU hardware to maximize throughput as the operations can largely be based on matrices.

CellModeller is mainly oriented towards studying cell growth, however due to the rule-based and ODE specifications, this simulator can reproduce a broad range of behaviors. Fractal patterns $\left[\mathrm{RFS}^{+} 13\right.$ and morphogenesis $\mathrm{NMDV}^{+} 17$ ] have been simulated with the help of CellModeller. 


\subsection{6 gro}

gro is a framework for cell programming and simulation. It includes a functional language and a simulator of growing rod-shaped bacterial populations in 2D. This platform offers a good GUI for visualizing the ongoing simulations. It also stands out due to its environmental signal intercell communication mechanisms. The software was developed at Klavins Lab, University of Washington. It is implemented in $\mathrm{C}++$, and composed by three parts:

1. A language for coordinating and controlling agents (CCL) Kla04]

2. A physics engine for handling mechanical interactions, Chipmunk LS]

3. The biological layer of the simulator, gro

This programming language is largely based on a rule-based paradigm consisting of guarded commands (rules of the form $G: C$ where $G$ is a Boolean condition that when satisfied, triggers the command block $C$, similar to an IFTHEN instruction). This freedom allows for an arbitrary level of abstraction in the implementation of cell behavior, since the rules can involve any variables present in the specification and can be as complex as needed. Two levels of rules are implemented in gro: environment level rules, which direct the general level of control in the simulation, and individual level rules that are responsible for the behavior of each cell.

Relating to mechanical processing of overlap, gro uses an algorithm implemented with the primitives offered by the physics engine Chipmunk for eliminating overlap between cells. The algorithm is effective, as cells are tightly packaged and well placed. However, this comes at a high cost, as the algorithm implemented in gro simply applies a very large number of iterations to adjust all new positions. The resulting simulation throughput is of 5000 bacteria in 15 minutes.

The biological layer establishes the dynamics and parameters for all of the biology elements involved in the execution (such as cells, environmental signals or the simulation space). Some examples include environmental signal diffusion and degradation dynamics (implemented with a finite element method) reactions between them or a default exponential growth model for cells and their division. Bacteria are treated as agents in the framework.

In June 2013, the source code of the software was released (and can be found at https://github.com/klavinslab/gro). Since then, the software has been used in many applications such as simulating finite state machines OK14, synthetic collective intelligence $\left[\mathrm{SADN}^{+} 16\right]$, morphogenetic developmental biology $\left.\mathrm{PPK}^{+} 16\right]$ and also by the 2016 Imperial College iGEM team in the Ecolibrium project (http://2016.igem.org/Team:Imperial_College).

This thesis has specifically built on gro, by proposing several new extensions to the platform and using it for simulating novel multicellular synthetic circuits using conjugation. This new version of the platform can be found at https://github.com/liaupm/GRO-LIA 


\subsubsection{Other AbM simulators}

All AbM simulators can be classified according to their focus and features. Some systems such as BSim $\mathrm{GMT}^{+12}$ and COMETS $\left[\mathrm{HRD}^{+} 14\right.$ are aimed at studying specific processes surrounding microbial populations. COMETS is oriented towards modeling the diffusion process associated with metabolites and how it affects bacterial populations. BSim is a 3D tool that seeks to study relationships between single cell dynamics and population behavior. A recently published simulator, Chaste $\mathrm{OFPF}^{+} 17$, is a cell-based system (equivalent of AbM in multicellular systems biology) concerned with studying tissue dynamics and development.

Also general-purpose frameworks, for studying microbial populations from different perspectives, have been developed. Biocellion [KKM ${ }^{+} 14$ is a general high-performance tool that runs on parallel computers and aimed toward the study of 3D multicellular biological systems. CeCe GL15] is a 2D general purpose microbial population simulator with continuous fluid dynamics capabilities for studying microfluidic systems.

\subsubsection{Programming frameworks for implementing AbMs}

Other solutions possess a lower level of abstraction, meaning they provide general programming frameworks for building AbM simulators. These frameworks are deemed multi-agent systems and provide a wide-range basis for programming entities that exhibit individual behavior and interact. Examples include REPAST [Col03], FLAME [KRH ${ }^{+} 10$, Netlogo [W04] and Mason $\mathrm{LCRP}^{+} 05$. All of these programming frameworks require agent behavior and environment rules definition. Mason and REPAST are Java-based multi-agent programming toolkits, FLAME uses XML definitions and runs on GPUs and HPC systems, while Netlogo is a multi-agent programming language in itself. 


\section{Chapter 3}

\section{Problem definition}

\subsection{Motivation}

Synthetic multicellular circuits using bacterial conjugation have not been extensively explored and constitute an interesting research direction. As mentioned in Section 2.1.4 this mechanism is a means for transporting a message richer in information within a local neighborhood between bacteria. The full biological process of conjugation is not completely understood yet. Therefore, a simulation tool to clarify the process and tinker with its parameters and dynamics would contribute in:

- Finding parameters for the simulation such that it reflects the conjugation process as realistically as possible.

- Simulating individual dynamics of the conjugation process to reproduce the effect observed in a bacterial population.

- Elucidating an algorithm for modeling conjugation

In addition of focusing on studying the process itself, using conjugation for constructing multicellular synthetic circuits is also a goal to pursue. Some simulators such as BactoSim, the IPS implemented by Krone et al., and DiSCUS are already capable of simulating bacterial conjugation to an extent. However, these systems do not simulate the whole effect of the plasmid migration associated with the genetic material being shared with a neighbor bacterium. This brings forward the issue of the simulation of gene expression in large scale colonies.

Combining long and short range communication mechanisms to generate more powerful synthetic circuits is also an interesting topic to study. None of the platforms currently supporting conjugation possesses more than a single mechanism. Conversely, other simulators displaying long range communication capabilities lack the implementation of bacterial conjugation. Thereby, a fast simulation tool that supports multiple communication mechanisms is needed. A related but different feature is nutrient consumption and cell growth. Krone's system and BactoSim are both capable of simulating these features. Nonetheless, the modulation of growth is limited, as these simulators represent bacteria 
as discrete entities performing a discrete version of growth and movement.

The main attention of the synthetic circuits to be simulated atop the new platform will be directed toward spatio-temporal patterns. Two requirements arise: 1) the tool should be fast enough to simulate a larger amount of bacteria than other simulators, and 2) it should have a good Graphical User Interface (GUI) to display the patterns at hand or any other circuit being simulated. This specific type of circuits (spatio-temporal pattern generating ones) would be a good proof of concept, as all of the simulator requirements are tested.

This thesis is motivated in finding novel designs and prototyping them with the help of a simulator that presents a new array of functionalities and allow for all of these features to be combined. The development of gro was driven by the need for an AbM to model and simulate multicellular circuits in the context of EU-FP7 project PLASWIRES (using plasmids as wires): http://www.plaswires.eu. This simulator should provide fast prototyping of synthetic multicellular designs and be easy to use.

\subsection{Research questions}

Developing new synthetic circuits is a difficult, long and costly process, as many hours are invested in thinking on the design, building the circuit and testing it in the lab. Also, the cost in time and money is further raised by the fundamental errors that may be found at the stage of construction and test of the circuits, as the experiments must be restarted and the lab material replaced. Much of this process could be shortened if enough information is made available at the implementation stage in the wet lab, and much of the trial process in the Build and Test phases of the development cycle would be eliminated.

An alternative to this approach is to use simulations for predicting (or prototyping) the behavior of a design, detecting errors and focusing on specific details of the experiment. Several classes of simulations exist within the scope of Systems and Synthetic Biology, being the most popular ones Ordinary Differential Equation (ODE) based, Partial Differential Equations (PDE) based, stochastic algorithms (such as the Gillespie algorithm) and AbMs.

Naturally, the reality cannot be fully reproduced in these simulations. To fulfill their role, simulators should reproduce a simplified reality and concentrate on accurately modeling a few key elements. Simulations shed light on several specific aspects that could take months to observe in a real experiment. They also help correct and refine the designs early in their development cycle. Information extracted from the simulations is meant to inform about certain parameters of the real experiment to the biologists carrying it out in the wet lab.

For simulators to be truly valuable, a certain (high) degree of realism must be preserved, and the focus should be as isolated as possible. The simulation realism can be associated with several features. In the context of this work, realism relates to how well a microbiology experiment in a Petri dish can be 
reproduced. This is, simulating a colony of bacteria. As shown in Section 2.4 , several frameworks to simulate this reality are already available. Unfortunately, these frameworks do not offer a toolkit complete enough for Systems and Synthetic Biology simulations to be carried out.

Some assumptions need to be taken into account when considering these simulators:

- The whole bacterial-environmental ecosystem cannot be simulated as it is way too complex and many of the intracellular, intercellular and environmental interactions are unknown or not well understood

- Computing power is limited and there is a trade-off between accuracy and scope (in terms of colony sizes)

- Physical simulated space is distorted and may not be an exact reconstitution of what happens in the Petri dish

Under these assumptions, a good simulator for studying multicellular synthetic genetic circuits should be able to reproduce enough intracellular, intercellular and environmental interactions. Specifically, on these levels, they refer to:

1. Intracellular: A general behavior model for each cell driven by gene expression

2. Intercellular: Short (bacterial conjugation) and long range (environment signals) communication mechanisms between bacteria

3. Environmental: Nutrient consumption for affecting growth rate of cells

It should also quickly reach a high number of simulated bacteria whilst being able to handle all of the mentioned interactions. Specific designs being proposed, a sufficiently good AbM tool should be able to simulate them. This should be as precise as possible within its physical space constraints. The aim of this work is to have the capacity for simulating complex multicellular genetic circuits with conjugation. It raises a first research question to tackle in this thesis:

RQ1: What potential do current AbM simulators have for simulating novel multicellular genetic circuits using bacterial conjugation?

A review of the existing simulators can elucidate this question and point out shortcomings. Overcoming these limitations yields a second research question:

RQ2: How should the identified shortcomings be overcome to enable the simulation of novel multicellular genetic circuits using bacterial conjugation?

This question establishes the basis for improvement and extension of a chosen platform and the requirements for simulating the proposed designs. This new platform should be used for simulating existing and novel multicellular genetic circuits that were not possible or were very difficult to simulate. Coming back to the original designs that need to be simulated, a platform meeting all 
requirements contemplated in RQ2 should be constructed. This is done by addresssing the shortcomings found in RQ1. The novel designs are to be simulated in this new platform, raising a final question:

RQ3: What novel designs relating to spatial and/or temporal patterns can the newly enhanced platform prototype?

This work will propose answers to the above questions.

\subsection{Methodology}

RQs direct whatever research is done to accomplish the goals of this thesis. These objectives can be summarized as:

- Studying AbM simulators and their capabilities for simulating novel multicellular circuits using conjugation.

- If no simulator can provide all of the needed features, improving and extending an existing one for it to comply with the requirements.

- Designing and simulating proposed novel circuits using the newly incorporated toolkit array in the platform.

Reaching the first objective is directly related to an analysis on the State of the art. A thorough study of AbM simulators was undertaken and literature on the topic reviewed (see Section 2.4). When this work started, not many AbM simulators were available, and even less were open source. gro [JOEK12, CellModeller [RSPH12, DiSCUS GMAdlC13, initial versions of BactoSim GRP15a and iDynoMiCS $\mathrm{LMM}^{+} 11$ were the candidates to fulfill the role of being the platform chosen to simulate new multicellular circuits.

BactoSim was initially considered a good choice, as it was one of the first simulators (along with DiSCUS) to implement bacterial conjugation. However, in BactoSim bacteria were represented as discrete static entities and had no support for gene expression. As these features were needed for the simulations, it was discarded as a possibility. iDynoMiCs had also been previously extended in the lab, but no documentation was available and the platform capabilities were very limited at the time. Also, the simulator worked mainly with spherical bacteria. The extension built in the lab was enabling iDynoMiCs for simulating rod-shaped bacteria. However, the use of this module slowed down the platform performance and conjugation was not present. In light of these pitfalls, iDynoMiCs was also discarded as a candidate.

The remaining simulators were the final candidates for initiating the task of extending the platform. At this stage, it was already clear that no existing simulator met all of the requirements, therefore answering RQ1. At this time, DiSCUS did not include conjugation dynamics or environmental signals (conjugation was added afterwards, but the code of this version is still not available) and CellModeller, in spite of being the fastest platform (it could simulate 32000 bacteria in 30 minutes) and offering a 3D environment, was not open source. 
CellModeller was released in 2015 to the public. gro was therefore selected as the simulator to extend. The choice was supported heavily by its good GUI and the programming language versatility for specifying circuits. Also, gro featured environmental signals as it hallmark, a valuable tool for intercell communication in the context of a 2D growing rod-shaped bacteria environment. Since gro was released to the public in June 2013, it proved to be the appropriate simulator to be extended.

Following the choice of gro as the starting platform, a roadmap of features to complement it was established:

- Improving the simulator speed

- Adding bacterial conjugation as an intercell communication mechanism

- Simplifying the gene expression process in the simulator and its specification

- Adding nutrient uptake and modulating cell growth

- Improving the environmental signal capabilities

The first problem that was identified is the simulator speed, in terms of bacterial counts. The bottleneck of gro was found to be in the physics engine. After changing parameters of the existing algorithm for handling physics and not getting a sufficient improvement in throughput, a decision was made to design and implement a new algorithm (the shoving algorithm of CellEngine). Initial simulations using both new features were tested and proved to be useful, as plasmid propagation simulations could be prototyped and bacterial counts reached orders of $10^{4}$ bacteria in under an hour.

Bacterial conjugation, being one of the original motivations behind the search for a simulation platform, was a core requirement. The mechanism was developed at the same time as CellEngine. Several different angles of the experimental process were studied, such as frequency-based vs. density-based conjugation or the parameters to reproduce realistic dynamics of the process. Data for the model fitting was taken from experimental papers published by Fernando de la Cruz lab $\left.\mathrm{dCRC}^{+} 12, \mathrm{FLDR}^{+} 14\right]$ and from data provided from the same lab in the context of PLASWIRES project. Specifically, generation times for bacteria (40 - 45 minutes) and conjugation rates ( 0.1 - 1.0 conjugations per cell and generation time) were the outcome.

As simulations scaled in complexity, it became increasingly difficult to specify and compute gene expression dynamics with gro's native language. This is when the need for a simplification and development of a new gene expression module (CellPro) and specification language layer (ProSpec) became evident. A scalable solution was required to this problem, so that specifying and simulating genetic circuits would not require as much detail and complexity as in the original version of gro.

Nutrient uptake and cell growth became issues when noticing from experimental data that bacterial colonies cease their growth after a lapse of time, 
but in a spatially unequal manner (growth stops in the center, but continues towards the colony edges $\left.\left[\mathrm{SLR}^{+} 12\right]\right)$. This functionality was concentrated in another new module, CellNutrient, using a Monod-based model to control cell growth with nutrient uptake.

Finally, the revision of signal capabilities was driven by the need to combine environmental signals and bacterial conjugation. This could be done with the signal capabilities of the original version of gro to a certain extent. As the simulations scaled in size, the existing signal capabilities became insufficient as the spatial area harboring the signals was bounded and the functionalities surrounding emission, absorption and dynamics of signals were limited. The development of CellSignals stemmed from these requirements.

The software development methodology used for all of the extensions except CellEngine, is a Waterfall Methodology $\mathrm{R}^{+} 70$. The development of each module followed a linear track in which sequential advances were made. However, they were mostly developed in parallel. CellEngine mimicked a spiral methodology Boe88 in its development. Once each module was done, it was then integrated into the gro simulator. Each of the developed modules answers the RQ2 question.

At this stage, and with a new platform providing novel features, complex multicellular circuits were prototyped and results were obtained from the simulations. Their design and simulation accounts for the answers to RQ3. The process of reaching final designs was cyclic in that an intital proposal was first prototyped and the flaws identified through simulations. This was possible since an expected behavior could be represented and compared to the results of simulations. Corrections were then made to designs until the expected behavior was reached. This process therefore exemplifies how the platform is useful as a tool for fast prototyping, early flaw detection, and parameter tuning.

Finally, and during the past 4 years, the new version of gro has been used to simulate multicellular biocircuits implemented in the lab by Fernando de la Cruz lab, and pertaining to PLASWIRES project. 


\section{Chapter 4}

\section{Proposed solution}

In this chapter, the solution reached by extending the gro platform will be explained. Also, novel synthetic circuits simulated with the platform will be described. Finally, a discussion will be provided. This discussion will refer to the choices made and the limitations associated to the outcome of every proposed feature.

\subsection{The first version of gro}

The first version of the software was released in June 2013 [Klaa. In its initial form, gro was a framework including a functional programming language for cells and a 2D bacterial colony simulator [JOEK12. gro was originally constructed through the integration of three components:

1. CCL Kla04, a guarded command specification language that is used in cooperative control systems. It is through CCL that the behavior of a cell is specified.

2. Chipmunk [LS], an open-source physics engine that is used to deal with the physical representation of entities in the simulator (cells). It treats bacteria as rigid bodies and calculates the collision physics in the environment, as well as handling their division.

3. gro, a module created to provide the biological background to the software. Within gro, all properties of the entities such as cells and environmental signals are stored and processed.

A summary of the framework architecture is shown in Figure 4.1 


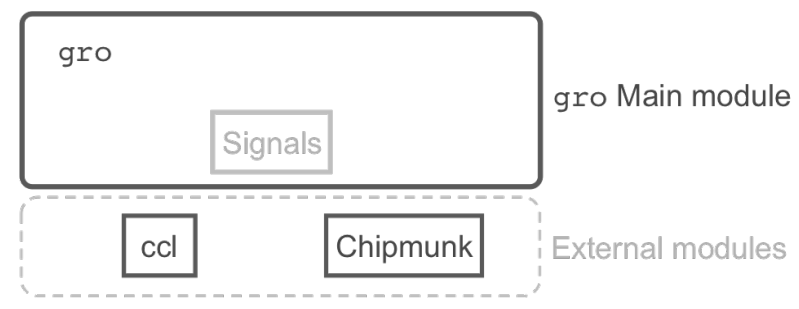

Figure 4.1: Original gro architecture. The system is composed of three modules: CCL, Chipmunk and gro. Both CCL and Chipmunk are independent subsystems that serve as agent control language and physics backbone respectively to the main module, gro.

gro's main utility is in simulating multicellular (synthetic) genetic circuits. Although a great deal of generality was possible within the framework, it was not able to quickly simulate large colonies. Specifically, the largest reported simulations with gro had around 84000 cells and took five days to simulate Klab. Despite this limitation, gro has been used to simulate synthetic collective intelligence $\left[\mathrm{SADN}^{+} 16\right]$, morphogenetic engineering $\left[\mathrm{PPK}^{+} 16\right]$ and RNA circuits [on].

Other shortcomings in gro include:

- Abstraction from genetic circuits to fuctional programs and their tedious implementation in specifications.

- Limited intercellular communication capabilites: environmental signals are the only form of cell communication and shows spatial limitations.

- Nutrient simulation is dependent on environmental signals and requires a large deal of configuration.

\subsection{A new version of gro}

The major focus of this work was to develop a more complete simulator to push forward the simulation capabilites in Systems and Synthetic Biology. To achieve this goal, gro was chosen as a starting point, as it is an open-source, extensible platform and has a reasonably good Graphical User Interface (GUI). The simulator extensions address the shortcomings of gro. As shown in Figure 4.1, the system is built on top of external modules that feed into the main gro module. In consequence, the extensions made for the simulator were also designed as external modules. A summary of the updated architecture is shown in Figure 4.2 and the general workflow of the system depicted in Figure 4.3. Each module will be further described in the following subsections. 


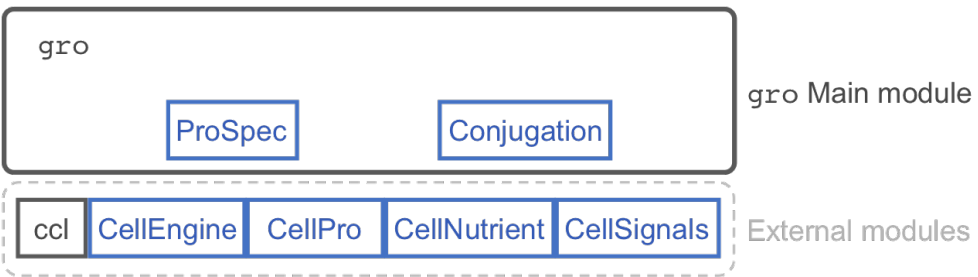

Figure 4.2: Updated gro architecture. In this architecture, several external modules (blue) have been added to newly include, extend or replace functionalities for the simulator. CellEngine replaces the previous physics engine Chipmunk. CellPro and CellNutrient are brand-new modules that handle protein expression and nutrient consumption respectively. CellSignals is an external module for extending the existing environmental signal primitives in gro. ProSpec was designed as an extension of CCL to provide a protein-oriented specification language layer. Finally, bacterial conjugation was implemented into the main module of gro.

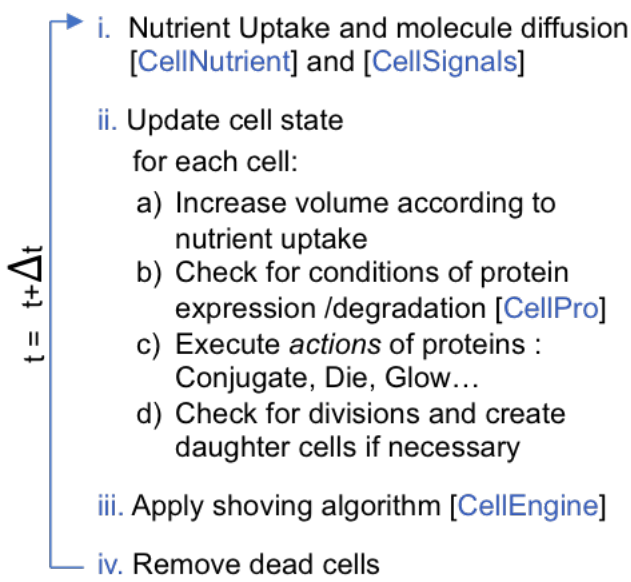

Figure 4.3: New gro workflow. All new modules are marked in blue and included in the operation of the workflow. Each iteration of the simulation goes through four stages: i) Nutrient uptake and signal diffusion calculations. These elements refer mainly to environmental values. ii) Cell state calculations. Every cell grows and updates its internal state. Actions are then executed under specific state conditions. Finally, cell division is evaluated and occurs if necessary. iii) Cell physics is calculated and are rearranged through a shoving algorithm. iv) Dead cells are removed from the simulation space. Reprinted with permission from Gutiérrez et al [GGGPdP ${ }^{+} 17$. Copyright 2017 American Chemical Society. 


\subsubsection{Improving physical engine speed: from Chipmunk to CellEngine}

The first step in making gro a better simulation platform was to get larger bacterial counts in colonies. A great deal of this improvement is directed towards designing and implementing a more efficient algorithm for computing the physics of growing bacteria in the colony. Throughout this section, the terms "(rigid) body" and "bacterium" will be used as interchangeable, since a general physics algorithm is described in the platform context.

\section{Chipmunk}

gro uses a shoving algorithm with Chipmunk as the backbone. Body overlap is generated at each simulation step between all bacteria in the colony when each of them grows. Resolution of this overlap is done through a cycle [MC95] in which a first stage detects collision between bodies Eri04 and then, the overlap is addressed [Mil07]. These operations are repeatedly applied to relieve pressure among bacteria until body overlap is inexistant. The algorithm is very accurate as it relocates bodies using very small differences in the body position. However, it is computationally costly since it requires hundreds of relocations to completely eliminate body overlap. This dynamics is depicted in Figure 4.4 .

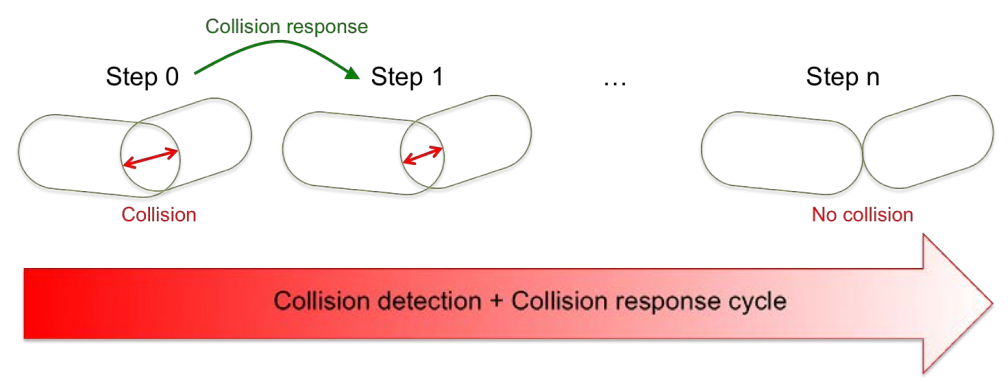

Figure 4.4: Chipmunk shoving dynamics in gro. Overlap between bodies is eliminated by moving them in the opposite direction causing the overlap. To accurately solve the collision, hundreds of steps are required.

Using this algorithm, the system becomes overwhelmed at about 5000 bacteria.

\section{CellEngine}

More efficient computation is required to achieve larger colonies. Several paths lie open to tackle this problem. One option is to vary the algorithm parameters (displacement size, number of iterations in the detection-response cycle). However, two facts make the solution a poor choice:

1. The displacement size does not allow for exact placement of the bodies with a similar precision: If the chosen size is too large, the array of bodies may end up either with excessive overlap or large gaps between them. If 
the displacement size is too small, the number of required iterations to solve the overlap would increase.

2. When adjusting the number of iterations, the computational cost is barely reduced and precision is also affected. A collision detection - response cycle still has to be executed for the bodies to organize, meaning a true gain in efficiency has not been attained. The reason for this is that the algorithm is $O\left(N^{2}\right)$ in computational complexity (where $N$ is the number of bodies). The solution applies to problems classified within the many-body problems type and their direct solution is of complexity $O\left(N^{2}\right)$ App85. Given the exponential rise in the number of bodies, a quadratic solution loses efficiency too fast.

Another solution is to design and implement a different algorithm to tackle the problem. This solution should have a lower complexity order and provide, if not the same accuracy, a good approximation. Quantitatively, this means that the density of colonies should be high (not many gaps between bodies and gaps should be small), and the angular orientation of nearby bacteria should be similar. Therefore, two assumptions are taken to develop the new algorithm:

1. The position and angular orientation of a body is strongly dependent on nearby bodies.

2. Bacterial colonies usually grow in an outward direction.

A new physics engine and shoving algorithm was designed and implemented by Luis Muñoz Mar14, GGGPdP ${ }^{+} 17$. The solution is called CellEngine and its operation is based on grouping bodies in ring structures and relieving pressure of the colony growth. Each body undergoes three steps: First, every bacterium is assigned a ring number, depending on the pressure it experiments (see Figure 4.5). Each ring has width a single body. This operation is performed inwards into the colony, as the outer bodies are the ones experimenting less pressure. Next, the resulting rings are grouped into sets of width $w$, and the individual bodies forming each group undergo a cycle of collision detection - response in the context of their own set. This phase is called relaxation, and accounts for the resolution of local forces, generated by nearby bodies (see Figure 4.6). This operation assumes that inwards sets of bodies cannot be moved (have infinite mass), but outwards sets are non-existant. Upon completion of this phase, new overlap arises with outward rings. At this stage, the third step of the algorithm, relocation, starts. The outer ring causing overlap is relocated in an outward manner while maintaining the contact points and relative positions with nearby bacteria (see Figure 4.7). Relocation seeks to approximate the effect of a distant, global force being exerted on each body coming from the colony center. Relaxation and relocation are executed until all rings have been processed by each of these steps. Upon completion of each iteration, the ring set is shifted one ring outwards, keeping its width of $w$ bodies. This algorithm is of computational complexity $O(N)$, as it performs each of the three steps (and $w$ times the two last ones) on each body. 


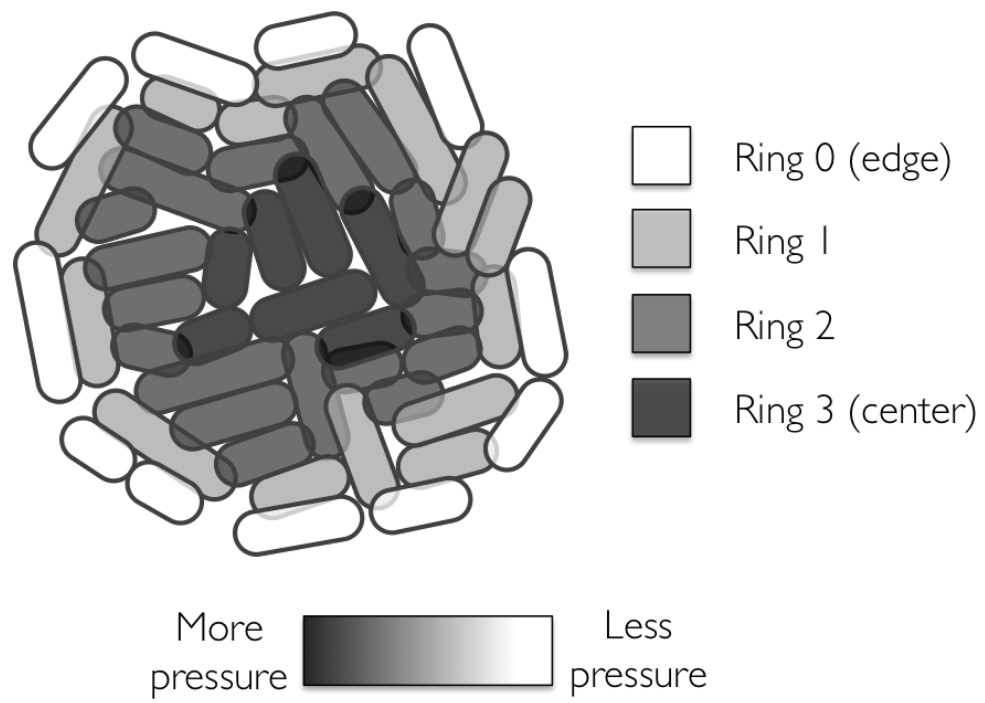

Figure 4.5: CellEngine ring assignation. Each bacterium in the colony is assigned a ring label $(l)$ depending on the pressure it experiments. The labels are assigned inwards. The first bacteria to get a label $(l=0)$ are those located in the outer edge of the colony. Next, whichever bacteria colliding with a labeled bacterium is assigned the label $l+1$. This is done until no bacteria remain without labeling in the colony.

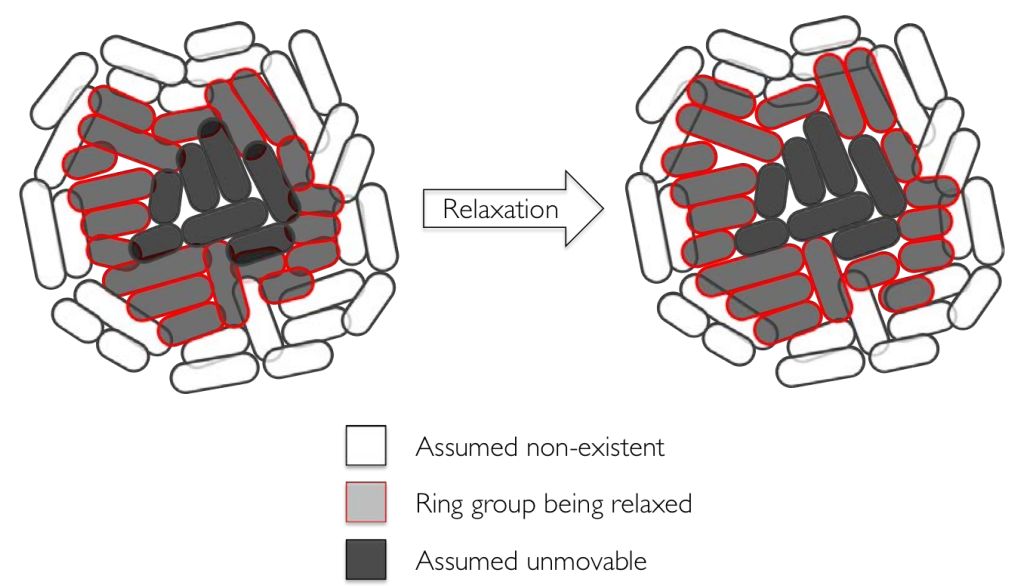

Figure 4.6: CellEngine relaxation step. A collision detection-response cycle is executed on the bacteria within a ring group to eliminate overlap in the group and also with the bacteria assumed unmovable. This part of the algorithm simulates application of local forces to each cell. 

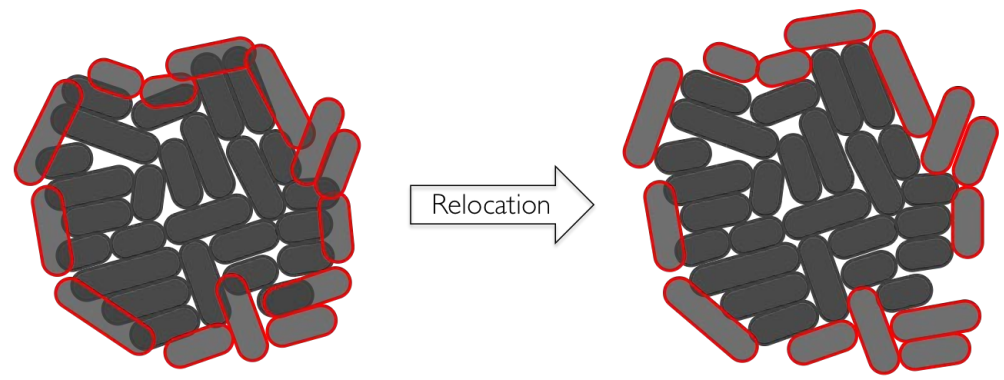

Ring group being relocated

Already processed ring groups

Figure 4.7: CellEngine relocation step. Cells in a ring group are moved outwards attempting to maintain all of the contacts they had when the algorithm iteration started. This step represents the application of global forces coming from the inner part of the colony.

This algorithm was implemented in $\mathrm{C}$ and replaced Chipmunk as the physics engine in gro. Performance of the system was tested and compared with the original version of gro (using Chipmunk). Also, the solution was tested for accuracy, by measuring the relative angular orientation and density of the colony. Results for both tests are shown in Figures 4.8 and 4.9 respectively.

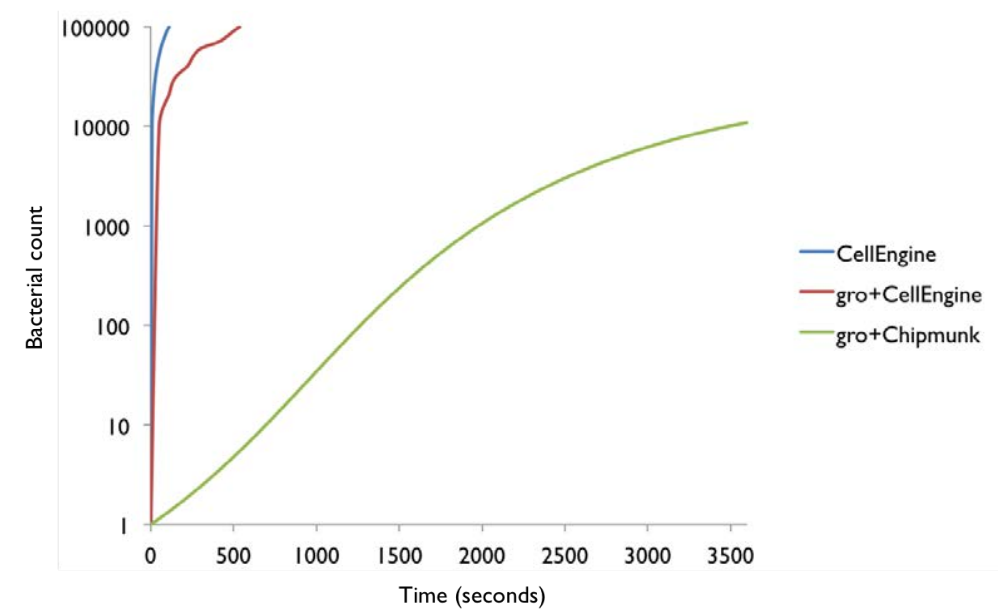

Figure 4.8: gro performance. A comparison of execution speed is compared between CellEngine alone, gro using CellEngine as its physics engine, and gro using Chipmunk. When CellEngine is used, $10^{5}$ bacteria can be simulated in a time under 10 minutes. The difference between CellEngine alone and gro using CellEngine lies in the parsing of the simulation being, and other modules being executed. To reach $10^{5}$ simulated bacteria, the original version of gro would take over a week: it reaches 84000 bacteria in 5 days. 


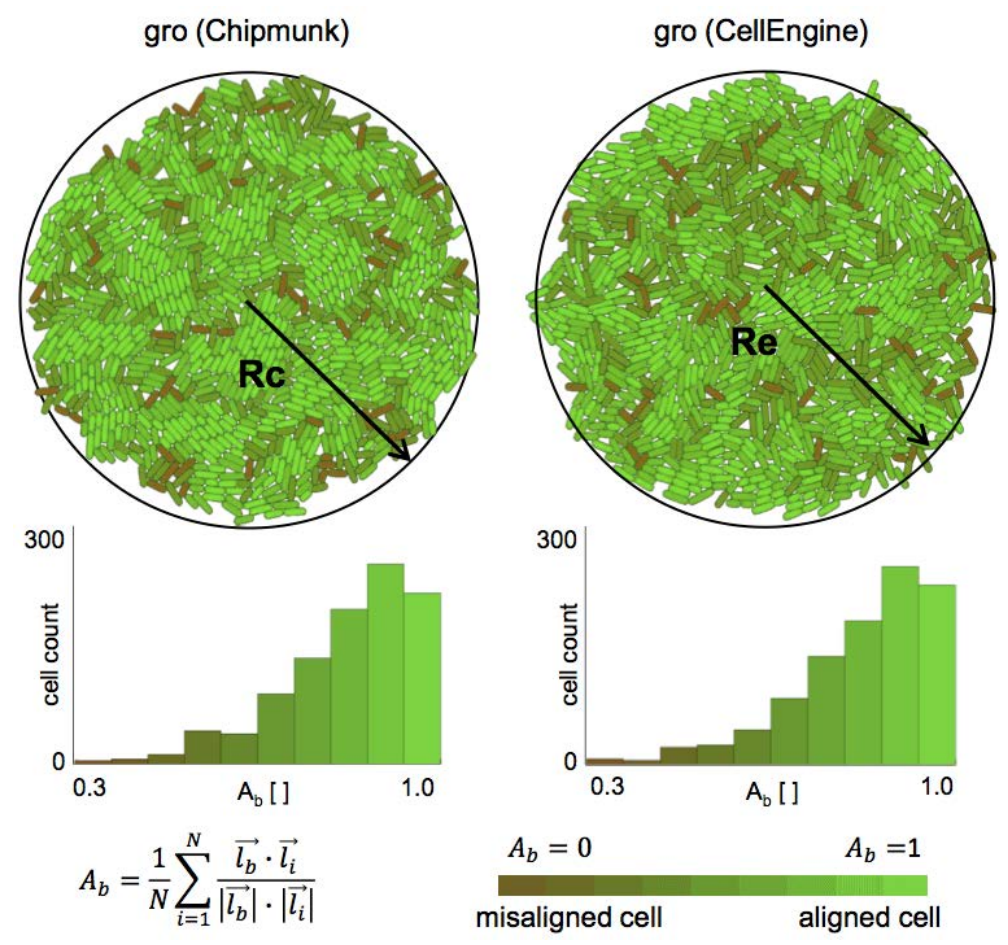

Figure 4.9: CellEngine validation. CellEngine is compared to Chipmunk in terms of cell relative orientation and colony density. $A_{b}$ represents an average of the relative orientation of a bacterium $\left(l_{b}\right)$ with respect to its neighbors $\left(l_{i}\right)$. As the figure shows, $A_{b}$ has very similar values when executing gro with CellEngine or Chipmunk. To compare density, the ratio $R_{c} / R_{e}$ was calculated, being $R_{c}$ and $R_{e}$ the colony radii obtained when running with Chipmunk and CellEngine respectively. $R_{c} / R_{e} \approx 1$ when comparing same colony sizes, showing the density is similar. Reprinted with permission from Gutiérrez et al GGGPdP $^{+} 17$. Copyright 2017 American Chemical Society.

\subsubsection{Enhancing cell programmability: from guarded com- mands to CellPro with ProSpec}

When designing a genetic circuit for Systems and/or Synthetic Biology, control of the biological "hardware" (bacteria within this framework) is needed. Programmability has to be represented and should be able to cover a large toolkit for the cell to use. The original version of gro offers a very general simulation framework in the form of a functional programming language, being the backbone for specification of cell behavior. This paradigm works very well if the person using the simulator is a computer scientist or otherwise skilled in programming. However, a different paradigm for specifying cell behavior is needed for making the simulation framework accessible to a broader audience. This is the motivation for the development of CellPro and ProSpec. 


\section{Guarded commands}

Cell behavior specification is done in gro through a guarded command (rulebased) language based on CCL Kla04. A guarded command is a rule of the form $G: C$ in which whenever a Boolean condition $G$ is satisfied (referred to as guard), it triggers the execution of an instruction block $C$. The paradigm translates to a structure composed of "IF $G$ THEN $C$ " rules that govern cell behavior. All data in the cell are codified as variables (numerical, Boolean, strings) that describe its internal state at any time. Every guard $G$ is a Boolean condition (simple or composite) involving these variables and some external values such as environmental signal concentration. Depending on whether $G$ is satisfied, the instruction block $C$ makes changes to the internal state and/or applies an operation on the environment such as signal emission.

This paradigm is very general and powerful since no restriction is imposed on $G$ or $C$. gro language is functional, and as such is an implementation of $\lambda$-calculus, which is Turing-complete Tur37. This assertion implies that a cell can be programmed to compute any function in the form of a $\lambda$-expression. However, a requirement to specify simulations this framework is to express the specification as a functional program. As much freedom as this paradigm provides, it still entails a necessary translation to variables and other abstract elements.

A gro specification with guarded commands generally has the following structure:

1. Library inclusion, parameter setting, definition of global variables and values, and function definition

2. Program definitions and operations

\section{Main program definition}

A simple example is shown in Appendix B.1. This specification describes a circuit in which cells sense an environmental signal (arabinose) and whenever it is found in sufficient concentration, the cell reports its presence by glowing in green. In this specification, the genetic circuit may also malfunction, yielding that either it glows forever or never glows at all. In this example, program $\mathrm{p}()$ codes the interal logic of cells, indicating when a cell must glow, when it must not and the conditions for malfunction. The reader can identify the guarded commands guiding cell behavior in the second section of the specification: "Program definitions and operations". The reader can also remark that every possible outcome must be implemented into the specification. This accounts for long specifications that although straightforward, force the programmer to code all possible outcomes and branches.

To better relate the specification to the biological reality and simplify the coding, a new paradigm was designed and implemented. 


\section{Protein-driven behavior and specification paradigm}

Bacteria undergo a process of gene expression, which ultimately produces proteins following the Central Dogma of Molecular Biology [Cri58, $\left.\mathrm{C}^{+} 70\right]$. Proteins are the main elements that drive cellular behavior Lod08. Following this assertion, protein expression can be made a central element driving bacterial behavior in the simulator specification. This involves two processes: 1) Gene expression simulation, and 2) Triggering of cell function based on the synthesized proteins.

Simulating gene expression is a complex task, as transcription and translation phases are involved and should in turn, be accurately reproduced. However, it is desirable that quick execution in the simulation environment is kept and that a simple underlying model simulates gene expression. To this end, a new module and a new specification language layer were designed and implemented.

The new module, CellPro, simulates gene expression under two key assumptions:

1. The whole process can be unified and simplified to time durations accounting for protein expression $\left(t_{a c t}\right)$ and degradation $\left(t_{\text {deg }}\right)$. A good choice for these times are the activation and degradation half-times respectively. Both of these times may of course suffer variability $\left(V_{a c t}\right.$ and $\left.V_{d e g}\right)$.

2. Proteins are assumed to be binary: it is expressed/present (taking a Boolean value of 1 ) or it is not (0).

Both of these assumptions are depicted in Figure 4.10

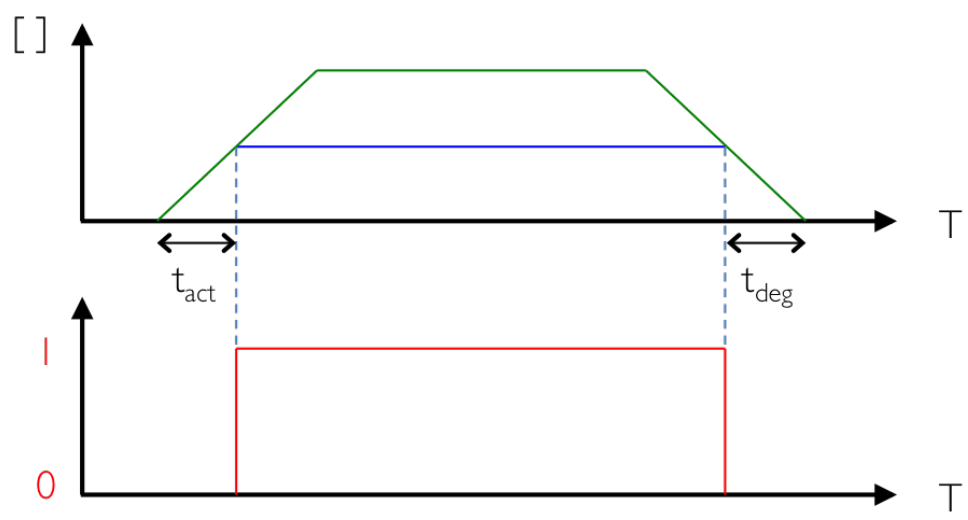

Figure 4.10: Protein expression in CellPro. The expression of a protein is assumed to be dependent on two parameters: $t_{a c t}$ and $t_{d e g}$, both corresponding to the half activation and half degradation time of the protein respectively (top). Whether a protein is being expressed or not is translated to a Boolean value (bottom).

Following these assumptions, several other elements are involved in the dynamics of CellPro. First, proteins are a resulting product of operons. Second, 
each operon is regulated by a promoter. Promoters are implemented as logic gates in which the inputs are presence of proteins (transcription factors) in the cell. Whenever logic gates (promoters) are triggered, activation timers for the protein expression start. Similarly, if the Boolean condition of the gate is not satisfied, degradation timers start running. Third, (permanent) anomalies in protein expression are included as percentages referring to a time. Within this timeframe, the given percentage of the population should be observed as failing. Finally, operons are included in plasmids, who in turn are the higher level genetic blocks representing cell programmability.

Each protein may be seen as a binary signal that is controlled by a noisy binary channel CLM11, represented by a promoter. The whole system translates to a Probabilistic Timed Automaton KNSS02, Sto02. Transitions in the automaton represent changes in the protein values related to the necessary time to switch from a protein value to a different one, along with a probability of a correct value switch. With all of these elements, a network of proteins is built that directs cellular behavior. Under given Boolean conditions based on protein values, cell actions are triggered. These conditions and the associated actions are evaluated/executed on an individual basis.

The codification of these elements in terms of guarded commands would stem a very complex specification, as the machinery would need a binary value per protein, a function for activating and degrading each of the proteins, a probability distribution function for handling abnormal operation, and a large set of rules involving the circuit logic and the triggering of cell function. In addition, the description complexity is variable, and depends on the circuit that would be simulated.

The process for gene expression was therefore condensed into CellPro as a set of bits (each bit representing the state of a protein) that change according to time, probability and values of other bits. This set of bits is organized as stated previously into operons and then in turn into plasmids, which can be held by individual cells.

The parameters needed to set a specification using CellPro have to be organized and packaged in an intuitive and clear way. This is why a new specification language layer, ProSpec, was created. The basic idea behind this language layer is to provide a syntax through which a programmer can specify a simulation structurally similar to how the genetic components of a microbiology experiment are organized. Plasmids and operons are the key elements involved in ProSpec. Proteins are defined as the input and output of operons. Plasmids then include a set of operons. Finally, plasmids are placed in cells. After the development of ProSpec, a related specification layer was published by Pascalie et al $\left[\mathrm{PPK}^{+} 16\right]$.

This new structure changes how the second part of a guarded command based gro specification is written. This part is now divided in two sections:

1. Definition and organization of genetic elements. This section defines the operative and parametric information of the circuit. Proteins are con- 
figured with their parameters $\left(t_{a c t}, t_{d e g}, V_{a c t}, V_{d e g}\right)$. These definitions involve the dynamics of proteins as outputs of an operon. Promoters in the form of logic gates (AND, OR, NOT, YES, ...) are also included in the operon specification and are configured by indicating the input transcription factors and, optionally, probabilities and times for permanent malfunction of the promoter. Once all operons are configured and set, they are placed into plasmids.

2. Definition of conditions for actions and their parameters. Cell function is driven by proteins, therefore a mechanism in which the existence of a protein in the cell triggers a specific reaction should be represented. This is done in ProSpec through Boolean conditions involving present, absent or "don't care" proteins. Whenever the Boolean condition is satisfied in a cell, an action is executed with its specific parameters.

To exemplify the shift in paradigm, the example of Appendix B.1 has been translated to a protein-driven form and is shown in Appendix B.2. The following differences can be remarked: First, the specification is more compact when in the protein-driven form. Second, the guarded command based specification handles many variables to implement the cell logic. Conversely, ProSpec based specifications hardly define any variables, yielding emergence of cell behavior uniquely in terms of involved genetic elements dynamics. Finally, while keeping the general order when specifying a simulation, protein-driven specification keeps a link to how an experiment should be described if taken to the wet lab. Specifically, all of the intracellular elements are organized in structures that are existent when dealing with DNA sequences (at the level of abstraction of genes).

In addition to the changes introduced by the new language layer and since proteins characterize the state of a cell, new data collection functions have also been constructed around the idea. Specifically, two new functions were added:

- It is now possible to screen an individual cell for all of its data in time (the dump_single directive): All of the cell physical and genetic information is extracted at every time step in the simulation and stored in a user-defined .csv file.

- The user may collect data concerning sets of bacteria satisfying Boolean conditions on proteins of the system. This directive, dump_multiple, takes in as input a set of Boolean conditions on proteins and a user-defined .csv file and stores into it the counts for all bacteria satisfying the conditions at every time step in the simulation.

Appendix A shows a complete reference for the syntax of ProSpec, summarizing what has been described in this subsection and providing a user manual for the new specification language. It is also available at: http://vps159.cesvima.upr.es/wpcontent/uploads/2017/05/ProSpecRef.pdf and https://github.com/liaupm/GROLIA 


\subsubsection{Extending intercellular communication: Conjuga- tion and signals}

One of the motivations driving the platform development was to have a framework capable of simulating cell colonies in which communication is a basic interaction amongst individuals. gro provided a set of commands for simulating environmental signals. However, other kinds of intercellular communication mechanisms were still missing from the toolkit. Horizontal gene transfer methods were the main focus, as vertical gene transfer are already implemented in the simulator. A couple of examples are bacterial conjugation and phage infection.

As mentioned in Chapter 2, many existing simulators could already reproduce communication among bacteria. Surprisingly, none of them includes multiple mechanisms nor integrates them with genetic function. In consequence, bacterial conjugation was chosen as the mechanism to implement into gro as a local communication method. Also, the existing signal directives in gro were revisited and extended to perform better and simulate a larger range of circuits than was possible until now.

\section{Bacterial conjugation}

Referred to as "bacterial sex", bacterial conjugation, or simply conjugation $\mathrm{KLF}^{+}$07, $\left.\mathrm{SGBF}^{+} 10, \mathrm{ZDF}^{+} 12\right]$, is a local transfer of genetic material from an origin bacterium to a neighboring destination bacterium. The material, in the form of a conjugative plasmid, is copied through a Type 4 secretion system $\mathrm{SGBF}^{+} 10$, GdlCR13] to the recipient bacterium. Based on what is known on the mechanical dynamics of conjugation on solid surfaces $\mathrm{KLF}^{+} 07, \mathrm{dCRC}^{+} 12$, FLDR $^{+} 14$, an algorithm was put in place to simulate the process. The proposed algorithm is evaluated at each time step of the simulation for bacteria which carry conjugative plasmids (see Figure 4.11 and Algorithm 4.1, reprinted

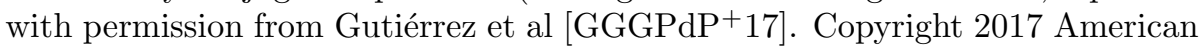
Chemical Society.). 


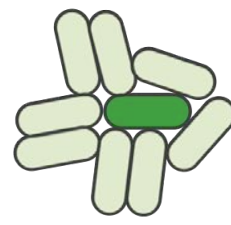

Initial colony

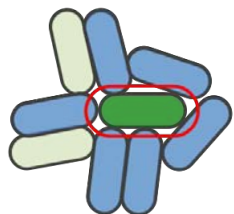

Neighborhood of the bacterium $\left(N_{b}=6\right)$
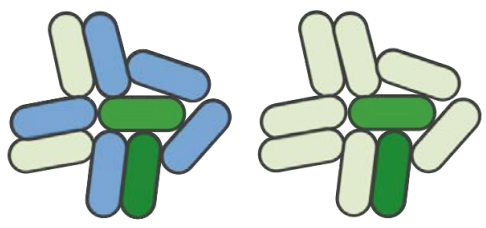

Randomly select one of the neighbors and copy pl to the selected neighbor

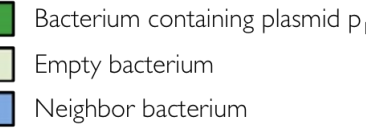

Figure 4.11: Simulating bacterial conjugation. The process is divided into three steps: 1) A bacterium carrying a conjugative plasmid $p_{1}$ checks its neighborhood for viable recipient bacteria. 2) A target bacterium is selected to be a host for the transferred plasmid. 3) Plasmid $p_{1}$ is copied to the target bacterium.

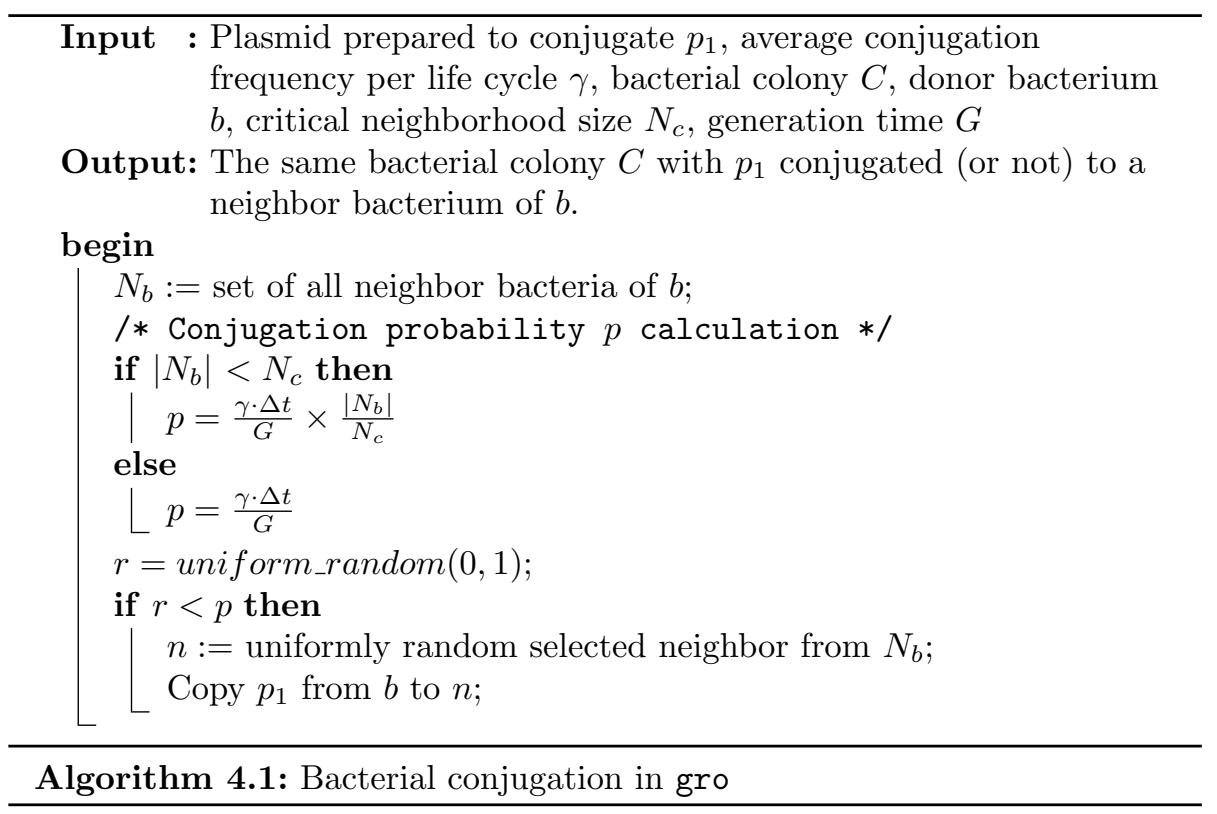

Algorithm 4.1 describes the logic of conjugation in gro, this being how and when a conjugative plasmid $\left(p_{1}\right)$ is transferred to a neighbor bacterium. Since conjugation is dependent on several factors such as time (frequency) and neighborhood (density), a probability of occurrence $(p)$ is calculated. A mixed method for conjugation was implemented to account for both factors. If $N_{b}$ is the number of neighbors a bacterium finds at a given time step, then the larger $N_{b}$ is, the higher $p$ will be. However, it is assumed that over a threshold, given by $N_{c}$, the bacterium likelihood for conjugation will be the same as in the threshold value. $p$ is also calculated based on the life cycle of the bacterium. Conjugation, being related to the cell metabolism, should also include time in its simulation. $\gamma$ is a conjugation rate that represents the average number of 
conjugations that take place during the time span of one generation time of the bacterium. Having calculated $p$, a random number $r$ is compared to $p$ and if $r$ is lower than $p$, a random neighboring bacterium is chosen and $p_{1}$ is copied to this neighbor.

Another conjugation-related mechanism was also implemented into gro in which the plasmid can be rejected by the recipient bacterium. This refers to the entry exclusion mechanism (EEX) GBdlC08. A list of excluded plasmids is held by each bacterium, and if an incoming plasmid is in this list, then it cannot be copied to the destination bacterium. In addition, a special conjugation directive was also included: it is called conjugate_directed and only calculates conjugation probability using competent neighbors. This is, it only uses neighboring bacteria in the calculation that do not have EEX against the plasmid to be transferred.

CellPro and ProSpec, being significantly subsequent to the implementation of bacterial conjugation, provide an ideal structured environment including all elements involved in conjugation implementation. Their design was actually guided by the previous versions of the conjugation implementations in gro. Furthermore, a consequence of including conjugation along with CellPro and ProSpec is its programmability. In Smillie et al. [SGBF ${ }^{+} 10$, necessary conditions for bacterial conjugation are stated, and plasmids are characterized as being mobilizable or conjugative. A mobilizable plasmid is one that includes incomplete machinery for conjugation, but that if provided with the missing elements may conjugate. A conjugative plasmid requires no further additional elements and thereby, can conjugate "at will". Both mobilizable and conjugative plasmids can be simulated with the platform. The case of conjugative plasmids is trivial and a direct application of the process described above. However, the case of mobilizable plasmids can be simulated by applying a Boolean condition of proteins on the conjugation action, therefore making it dependent on the presence of required proteins. Since CellPro and ProSpec consider a hierarchical organization of the genetic elements, this rule extends to foreign plasmids that could produce the missing requirements for conjugation.

Conjugation was implemented directly into the gro source code, making it possible to use either as an instruction for guarded commands or as an action in the context of ProSpec.

\section{Revisiting environmental signals}

gro is a good platform for simulating growing bacterial colonies that communicate through environmental signals. It is in fact one of the signature features of the first version of gro. As the platform was being extended, the need for environmental signals to be included in genetic circuits arose. The built-in module from the original version of gro was used in new circuits being simulated. However, the provided implementation proved to be insufficient since colonies now reached larger sizes. Environmental signals implementation had to be revisited as it needed to adapt and scale along with the colony sizes. Area limitation for a signal is shown in Figure 4.12 . 


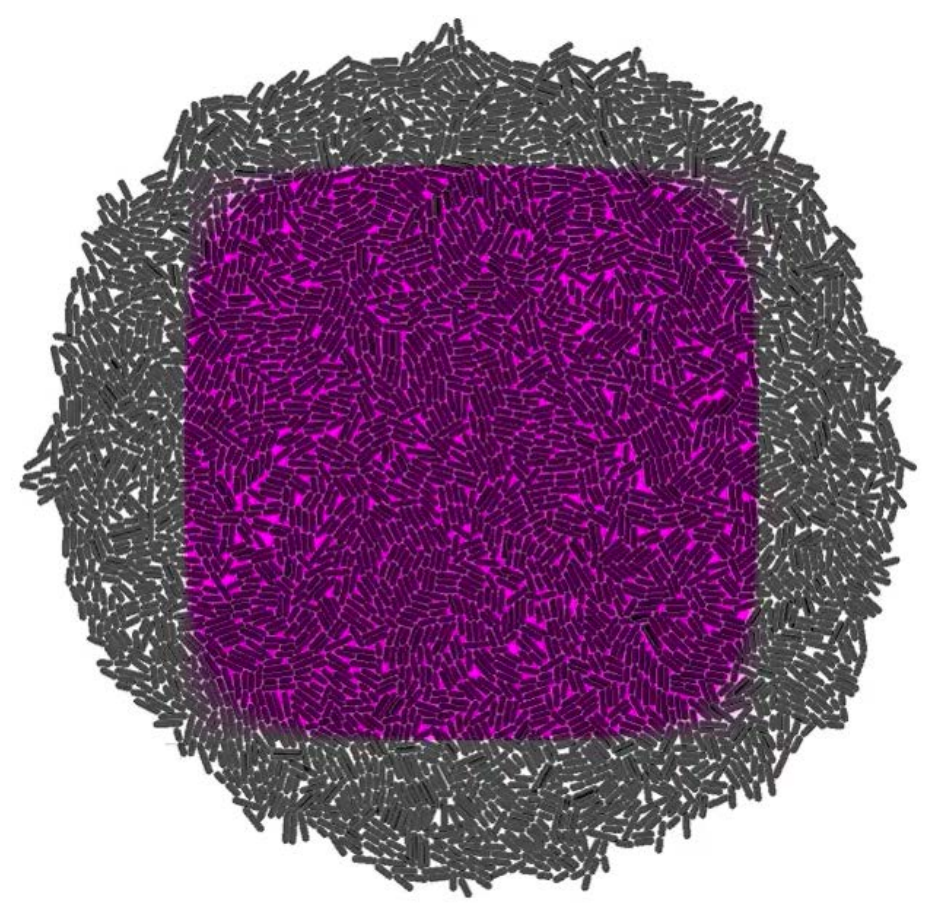

Figure 4.12: Signal area limit. The original implementation of signals in gro is limited to a static grid size. In this simulation, cells are gray in color and the environmental signal is colored in magenta. This figure shows a diffusive environment signal and how a bacterial colony outgrows the valid grid zone as it does not rescale. The signal hence retaining its maximum size within the square grid.

The original version of signals uses the finite element method DR82, RG10 to simulate diffusion. This algorithm applies discretization over the grid and integrating with the Euler method [JOEK12]. Its operation is represented in the form of a fixed coefficient matrix that traverses all of the signal grid cells and provides the template for diffusion operations (Euler integration) on the current grid cell. The default matrix, $M_{d i f}$, of dimension $3 \times 3$ used by gro is:

$$
M_{\text {dif }}=\left[\begin{array}{ccc}
0.5 & 1.0 & 0.5 \\
1.0 & -6.0 & 1.0 \\
0.5 & 1.0 & 0.5
\end{array}\right]
$$

This method results in a fast diffusion in the colony environment. Also, several signals can be processed at each iteration since one grid is used per signal.

Since the mentioned limitations needed to be addressed, an opportunity to globally enrich the existing signal implementation was taken. Specifically, the following additional shortcomings or possibilities were identified: 
- The diffusion process can only be adjusted through a single parameter (diffusion constant). More flexibility is needed for this parametrization.

- Signals in gro are analog in nature. How would a digital version be implemented? What for?

- Bacteria do not always emit/sense signals at a fixed point in their geometry. More approaches are needed to model signal emission/sensing.

Each of the items gives rise to a feature that can be implemented into the solution. Consequently, the features were designed and implemented into an external module called CellSignals. A brief description of each improvement or novel addition will be shown below.

1. A dynamic grid to reflect a larger surface of simulated bacteria was implemented. The initial size of the grid can be set and so can the size of each grid cell. However, when the space needed for further signal diffusion becomes insufficient, the grid resizes allowing continuity in the diffusion process. This event takes place whenever the signal reaches a close neighborhood of the grid border. A simulation using this new feature is shown in Figure 4.13.

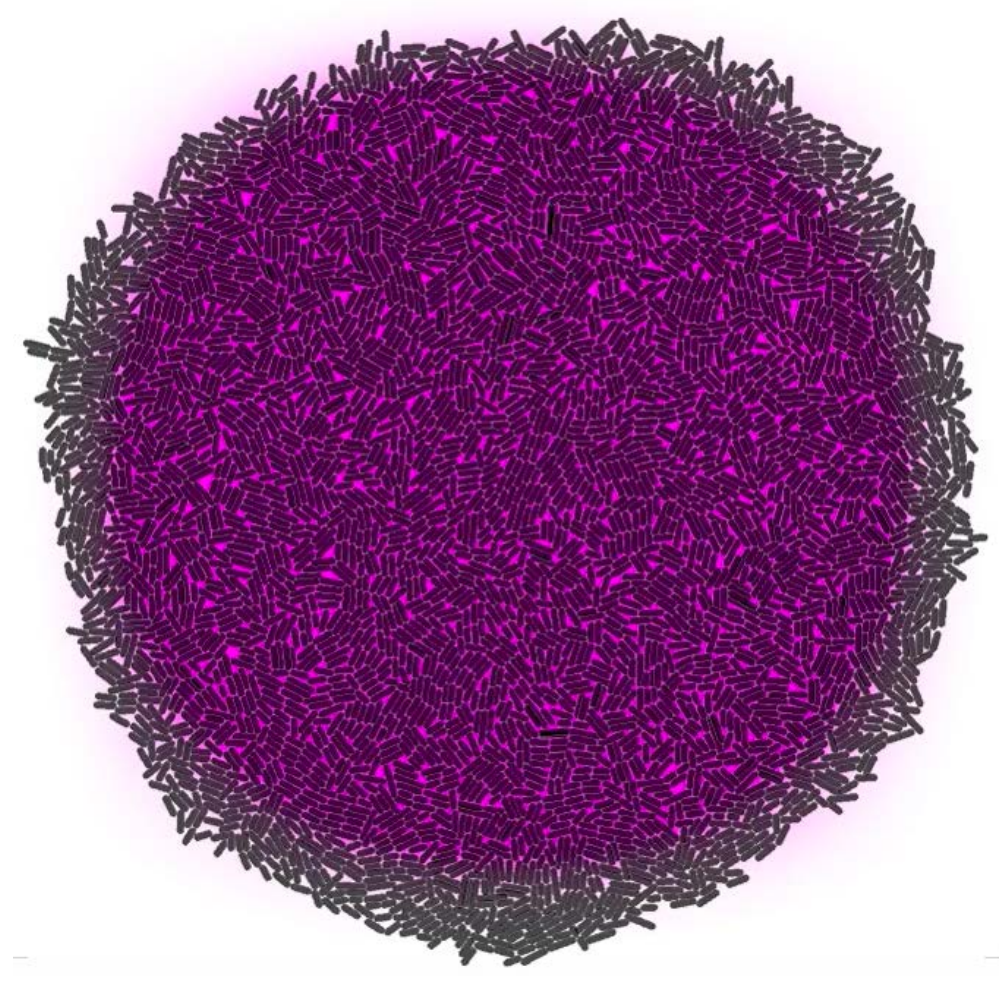

Figure 4.13: Dynamic sized signal grid. In this figure, cells are colored in gray and the environmental signal is magenta. The cell colony grows and the signal diffuses in the environment. Unlike Figure 4.12, this simulation uses the resizable grid and therefore does not limit signal diffusion. 
2. Integration coefficients for the Euler method are organized in a matrix mask of size $3 \times 3$ grid cells. These coefficients were originally fixed, and diffusion could only be adjusted through the external constant. Coefficients of the integration matrix mask have a direct effect on the direction in which the signal diffuses. Specifically, they determine from where the central grid cell acquires signal concentration with respect to its immediate neighbors. In this context, a programmer can now provide custom values for the matrix mask to direct how diffusion should behave spatially. Figure 4.14 shows the effect of custom matrices on signal diffusion.

Figure 4.14: Custom integration matrix. This example depicts the emission of four different signals, each one having its own custom integration matrix.

3. To align with the binary philosophy surrounding the system, a different kind of signals were implemented. Unlike the analog version, digital ones are represented by a single value at a grid cell: whether the signal is present in that location or not. This kind of signal handles diffusion and degradation in a different manner: digital diffusion is modeled as a particle transference between grid cells, and digital degradation occurs after a counter reaches a threshold. Emission of digital signals is shown in Figure 4.15 


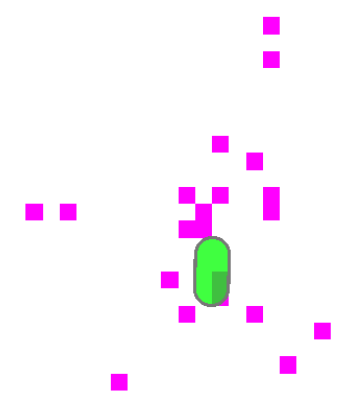

Figure 4.15: Digital signals. Emission, diffusion and degradation of a digital signal (magenta).

4. Improving speed was also a concern, although secondary. To gain in speed, all of the signals were concentrated into a single grid and diffusion for all signals in the simulation can be calculated in only one pass over the grid.

5. gro provided an interface for signal emission and absorption that was limited to the center location of a cell. Now, other possible spatial distributions for emitting and absorbing environmental signals have been added. These distributions are exact, area and average. exact distribution is the original one, in which signal is processed at the center grid cell of the bacterium. area processes a given amount of signal at all of the grid cells covered by the bacterium. average mode equally distributes a concentration value of a signal over all of the grid cells covered by the bacterium.

In sum, CellSignals extends the functionality that gro already provided for larger bacterial colonies and also includes new options for controlling and defining environmental signals. ProSpec directives for using these new signal functionalities are summarized in Appendix A

\subsubsection{Nutrients: CellNutrient}

Another functionality that could be simulated with gro, but required substantial control from within the simulation implementation is nutrient uptake along with its effect on cell growth. Natively, gro simulates cells growing at an exponential rate (see Eq. 4.1, being $V$ the bacterium volume and $k$ the growth rate), translating into an assumption of infinite nutrients.

$$
\frac{d V}{d t}=k V
$$

However, with the use of signals, it is possible to emulate nutrient dynamics and modulate the speed at which cells grow. It is, nonetheless, a tedious exercise 
as it requires to keep track of signal concentration and adjust growth rate according to the sensed amount of signal. In addition, no definite nutrient-based growth model is defined, therefore requiring an implementation of the whole model from scratch.

To make the use of nutrient uptake and its associated cell growth more accessible, an external module called CellNutrient Sáe15 was implemented. It follows Monod equation Mon49] (Eq. 4.2) for relating nutrient uptake to cell growth.

$$
k=k_{\max } \frac{S}{S+\kappa}
$$

In Eq. 4.2, $k$ is the growth rate of the bacterium and is a function of $k_{\max }$ (its maximum growth rate), $S$ (available amount of nutrients at the bacterium location) and $\kappa$ (half saturation constant). The nutrient concentration is stored in a grid, pretty much as in CellSignals, and is also dynamic in size. The operation algorithm for including Eq. 4.2 is shown in Algorithm 4.2, taken from Gutiérrez et al. GGGPdP ${ }^{+} 17$. Parameters $S$ and $k_{\max }$ can be set by the user as global parameters of a specification. An example showing how this is done can be found in Appendix A.

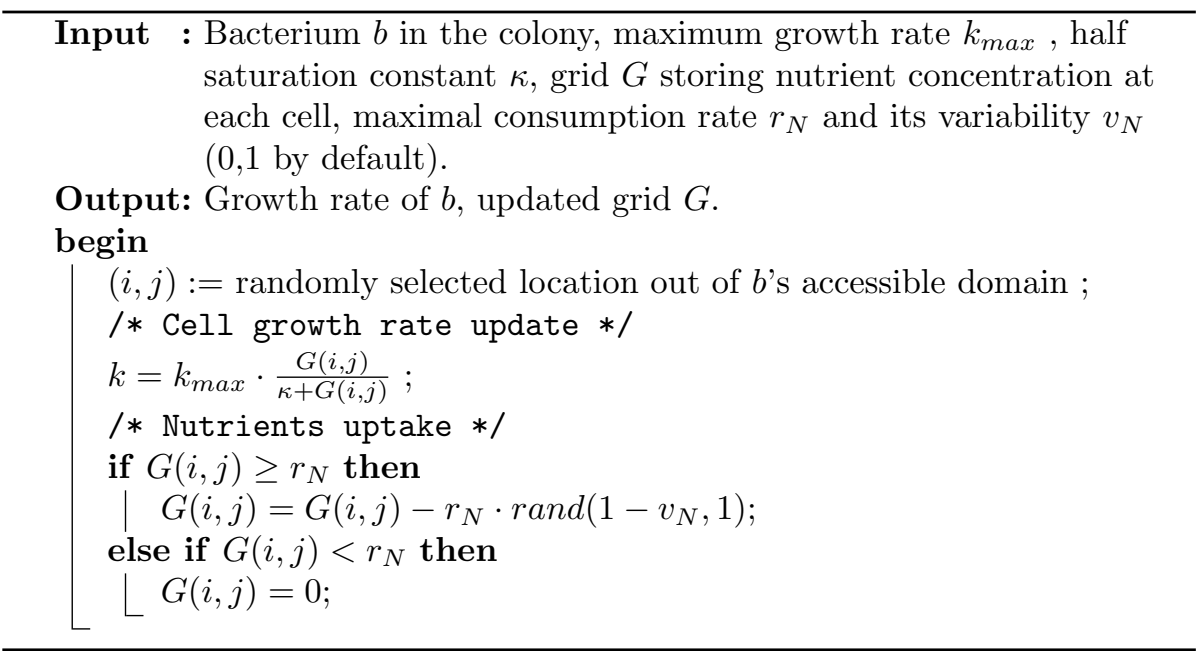

Algorithm 4.2: Nutrient uptake.

Algorithm 4.2 describes the process of nutrient uptake and bacterial growth. First, the bacterium (b) randomly selects a grid location from where it will consume nutrient. Next, it calculates its growth rate based on Eq. 4.2, where $k_{\text {max }}$ is the parameter ecoli_growth_rate of gro, $G(i, j)$ is the nutrient concentration at grid cell $(i, j)$ and $\kappa$ is an internal parameter of the module that accounts for the half saturation constant. Finally, nutrient concentration is recalculated: If the available concentration of nutrients at $(i, j)$ is greater than the maximal nutrient consumption rate, then an amount of nutrients is subtracted from $G(i, j)$ following an uniform probability distribution. Else, if the amount of nutrients at the location are limited and lower than the nutrient consumption 
rate, whatever concentration left is consumed.

To illustrate these concepts, Figure 4.16 depicts a summary of how colonies grow with different nutrient distributions and concentration amounts using CellNutrient.

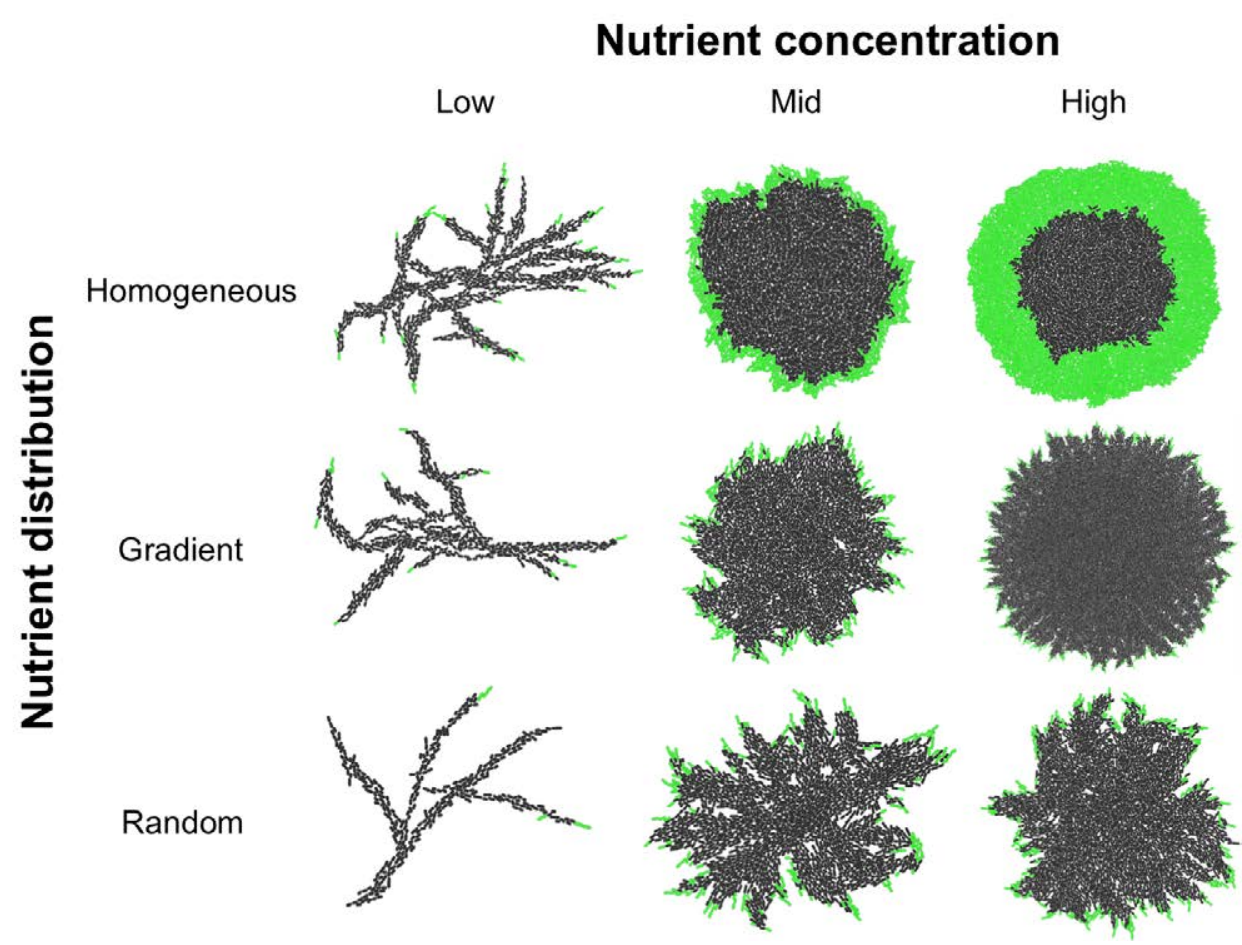

Figure 4.16: Colony growth with nutrients. Growing bacteria are colored in green, while those that are not bear a dark gray color. Each combination of nutrient distribution/concentration yields a distinct grotwh pattern in bacterial colonies. A higher concentration of nutrients causes bacteria to grow for a longer time, hence an outer ring of bacteria growing arises. The higher the concentration of nutrients, the thicker the growth ring is. Low concentrations of nutrient generate filamentous shaped colonies. Distribution affects in the density while bacterial colonies grow: Homogeneous distribution preserves a high density of bacteria, being the amount of nutrient uniform over all of the simulation space. Gradient distribution desposits a descending amount of nutrient the further the location is from the simulation space center that causes the filaments to be observed once the quantity of nutrients is low. Finally, random distribution assigns an arbitrary amount of nutrient at a given location, capped by a given threshold. The latter distribution shows the lowest bacterial density while the colonies grow.

By using CellNutrient, gro is able to reproduce colony morphologies shown in Tokita et al. $\left[\mathrm{TKM}^{+} 09\right]$ and follows a linear radial growth speed $\left[\mathrm{DTD}^{+} 14\right.$, LW81, FHMW13. 


\subsection{Novel multicellular circuits}

In this section, several designs that were simulated on the presented platform will be shown. The idea of this section is to view the platform in action and discuss results and limitations that were extracted from the simulations run in the new version of gro. These circuits rely on the novel additions that were made to gro, but also confirm usefulness of the toolkit, as new results can be simulated.

\subsubsection{Spatio-temporal patterns}

gro is a simulator that is capable of prototyping genetic multicellular circuits. Spatial arrangements can be observed in simulations due to its $2 \mathrm{D}$ graphical interface. Considering that the improvements described previously have made the framework scale to larger extents and its toolkit has been expanded, more complex designs making use of these features can be tested. Novel circuits were prototyped using the presented version of gro. The expected outcome of these designs was for them to exhibit spatio-temporal patterns in the cell arrangements.

\section{Bullseye patterns}

In Chapter 2, a circuit by Basu et al. $\mathrm{BGC}^{+} 05$ was introduced. Basu's circuit, for detecting a band of AHL concentration, is an intracellular circuit. Both sender and receiver bacteria have a fixed task and perform it on their own. Sender bacteria act through the $P_{\text {sender }}$ logic, while receivers use both $P_{\text {high }}$ and $P_{l o w}$. Inspired on this circuit, two novel bullseye pattern circuits were developed, and a third one was designed as a proof of concept for scalability. The desired resulting patterns are shown in Figure 4.17
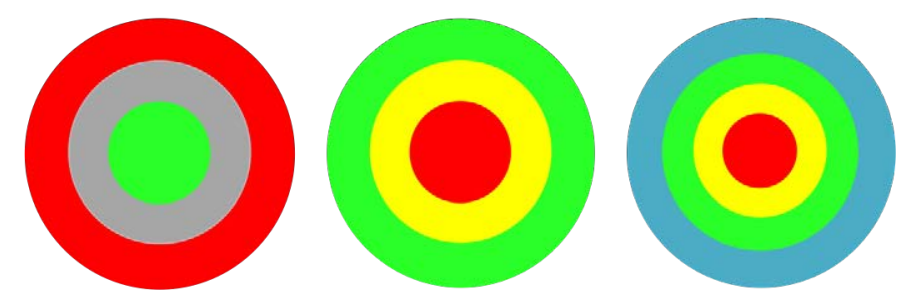

Expected results for the bullseye circuits

Figure 4.17: Expected results for bullseye pattern circuits. Patterns of two, three and four levels are the goals to achieve with the design of novel circuits. These patterns are respectively depicted form left (two-level) to right (fourlevel).

First, a multicellular circuit for generating a two-level bullseye pattern in which a band for mid-concentration is also present, but does not produce any action. Instead of being driven by AHL concentration, an IPTG signal cue serves as the template. Two plasmids detect low and high concentrations of 
IPTG, like in Basu's circuit. However in this case, whenever each plasmid is in the zone of concentration it should detect, it amplifies by activating its own conjugation in addition to reporting (high concentration of IPTG is reported with GFP - Green Fluorescent Protein by $P_{\text {high }}$ while $P_{\text {low }}$ reports low concentration with RFP - Red Fluorescent Protein). Also, bacteria containing each of the plasmids are well-mixed. In Basu's circuit, $\operatorname{lux} R$ was tuned to activate with different thresholds of detected AHL. In the present circuit, sensitivity is achieved through the effect of IPTG on lacI. A variant of lacI ( lacI $_{2-1 A 5}$ [LR09]) that is more sensitive to IPTG, and the regular version of lacI were used in the design. This allows the activation of lacI under different concentrations (thresholds) of IPTG. 


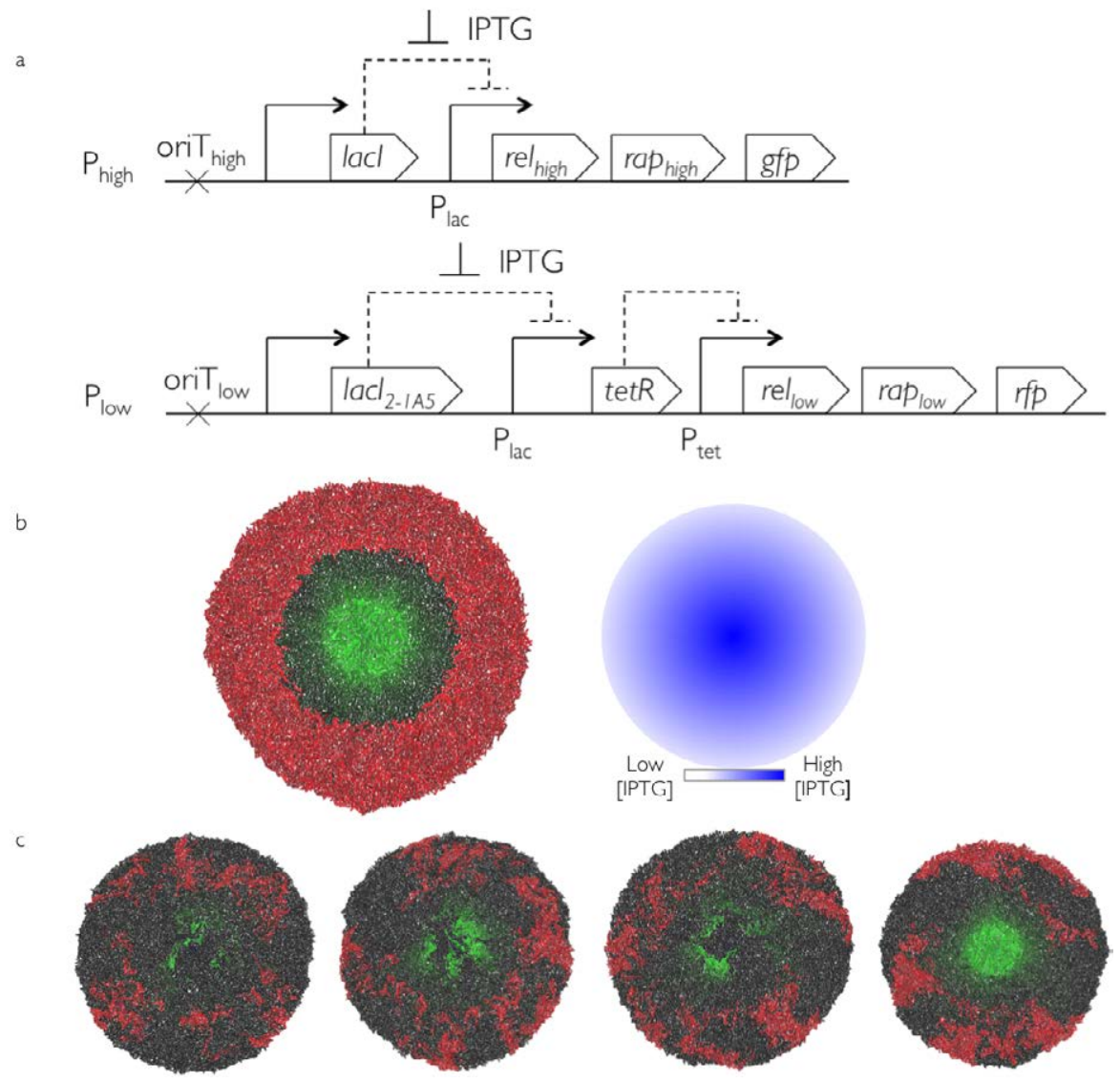

Figure 4.18: Two-level multicellular bullseye pattern (figure partially taken from Gutiérrez et al $\mathrm{GGGPdP}^{+} 17$. Reprinted with permission from Gutiérrez et al $\mathrm{GGGPdP}^{+} 17$. Copyright 2017 American Chemical Society.). In subfigure a), the circuit that implements the pattern is shown. Two plasmids $P_{\text {high }}$ and $P_{\text {low }}$ establish IPTG thresholds for reporting and conjugating. Relaxases ( $\mathrm{rel}$ ) GBFdlC09 and Relaxase Accessory Proteins ( $\mathrm{rap}$ ) [LGRCC02, Chr04, $\left.\mathrm{CRRP}^{+} 15\right]$ are key proteins involved in conjugation. Their expression is controlled by the detected amount of IPTG. Subfigure b) shows a pattern generated by the circuit with a very high conjugation rate (20 conjugations per life cycle) along with the IPTG gradient giving rise to it. Finally, subfigure c) demonstrates how the circuit outcome varies in relation to conjugation rate. The rates used are respectively $0.1,0.5,1.0$ and 5.0 conjugations per life cycle. Further details can be found in the source code, shown in Appendix B.3.

The parameters that affect the circuit outcome are conjugation rate, lacI and $l a c I_{2-1 A 5}$ sensitivities to IPTG and GFP/RFP activation and degradation times. Conjugation rate relates to how dense the reporting of an area is. The higher the conjugation rate, the better the zone related to this rate will be reported. Figure 4.18 shows that at higher conjugation rates, more uniform colored (red and green) and uncolored zones are achieved. Sensitivities of the 
lacI variants translate to the width of detected zones. In practice, only green (high) and uncolored (mid) zones width can be varied, since the red (low) zone only requires a start IPTG concentration threshold as it detects and reports all locations with IPTG concentration below that threshold. Finally, activation and degradation times of $G F P / R F P$ also relate to the reported zone width. Degradation time is the most relevant one for $G F P$, while activation time is the one having most perceivable effects on $R F P$. This is because GFP degradation time marks how fast reporting dilution occurs, while activation of $R F P$ marks how soon the low concentration zone is reported.

The second bullseye pattern that was developed is a multicellular one marking three different zones. The circuit detects three zones of IPTG (high, mid and low), through a plasmid for each zone. Segregation in this circuit not only occurs by reporting, but also by killing bacteria. Activity of a plasmid in its intended zone is composed by conjugation, reporting, cell survival, and in specific cases, signaling. This design is scalable on the number of orthogonal signals used to communicate position in the area. Figure 4.19 depicts the circuit details and its outcome from simulation. This design is more complex than the two-level bullseye one and involves using a larger amount of tools and mechanics such as cell death, integrase-based flipping and quorum sensing (QS) communication methods. 


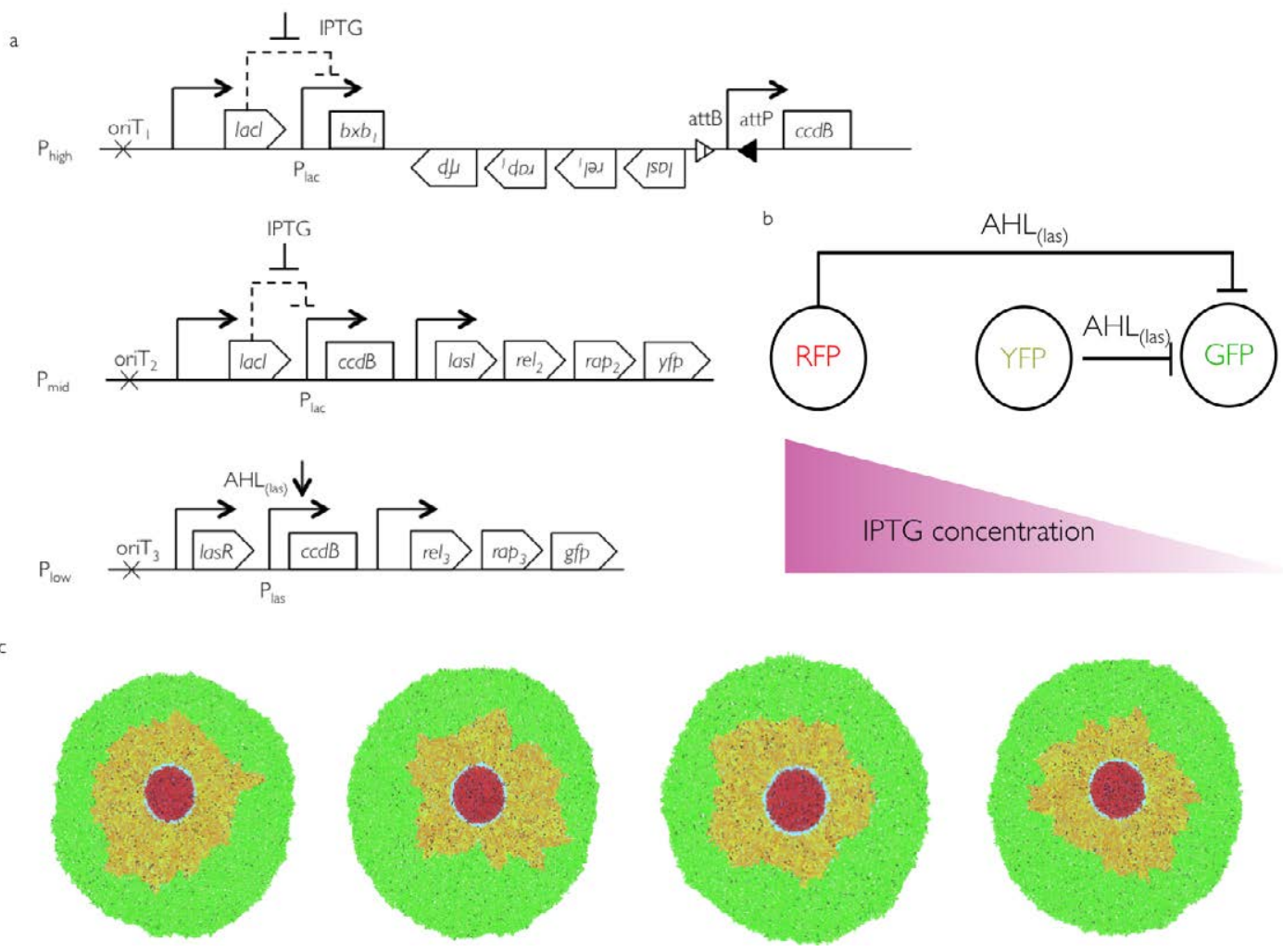

Figure 4.19: Three-level multicellular bullseye pattern (figure partially taken from Gutiérrez et al $\mathrm{GGGPdP}^{+} 17$. Reprinted with permission from Gutiérrez et al GGGPdP ${ }^{+}$17. Copyright 2017 American Chemical Society.). Subfigure a) shows the circuit design. Each plasmid acts in a zone with a given IPTG concentration (high, mid and low). If the plasmid is not in its detection zone, $c c d B$ toxin is activated and kills the cell. $P_{\text {high }}$ plasmid conjugates, emits $A H L_{(\text {las })}$ and expresses $R F P$ if the concentration of IPTG is sufficiently high. Otherwise, $P_{\text {high }}$ triggers cell death through $c c d B$. These conditional actions are selected by $b x b_{1}$ integrase [BSE12], that flips transcription orientation. $P_{\text {mid }}$ conjugates, emits $A H L_{(\text {las })}$ and expresses YFP (Yellow Fluorescent Protein) outside the high IPTG concentration zone. Whenever $P_{\text {mid }}$ detects a high IPTG, it initiates the expression of $c c d B$. $P_{\text {low }}$ constantly conjugates and expresses $G F P$, however, if it detects $A H L_{(\text {las })}, c c d B$ is expressed. Subfigure b) depicts the relation among different zones of the pattern caused by environmental signals IPTG and $A H L_{(\text {las })}$. Subfigure c) shows different executions of the circuit in gro after 300 simulated minutes. The three zones are distinctive - the red area is in the center, where high IPTG is detected. The yellow zone is located outside the red one, and the outer crown is a green one. The full source code for this simulation can be found in Appendix B.4.

Execution of the presented circuit is sensitive to IPTG levels and their detection, bacteria type initial ratio and their growth rate, and cell death speed. IPTG concentration directs what size the inner $R F P$ core has and hence, the 
$Y F P$ zone initial border. It is important to note that GFP is not directly related to IPTG concentration, but depends on where $R F P$ and $Y F P$ are not present. Initial quantities of bacteria carrying each plasmid are also important parameters, as in a random distribution of the cells, they determine the likelihood of a bacterium operating in a zone it is intended to act. Growth rate is an important parameter, since GFP expressing cells have to be capable of outgrowing $Y F P$ ones ( $G F P$ bacteria are killed by $Y F P$ expressing ones) to form the outer crown of the pattern. In this sense, $P_{l o w}$ carrying bacteria must be the fastest growing ones. Finally, it is important to control the correct placement of bacteria in their respective zones by having a timely death of those bacteria not located in their intended zone.

The proposed circuit is scalable to the number of zones by replicating the logic of $P_{m i d}$ with a different diffusible signal being emitted and the signaling particles coming from the "previous" plasmids causing death to the cell carrying the current plasmid. This scalability is also a limitation as not many orthogonal signaling systems have been found. However, a recent paper by Scott and Hasty SH16] describes signaling systems that can be used to these ends. The outcome of a prototype for the circuit reporting four zones is shown in Figure 4.20.

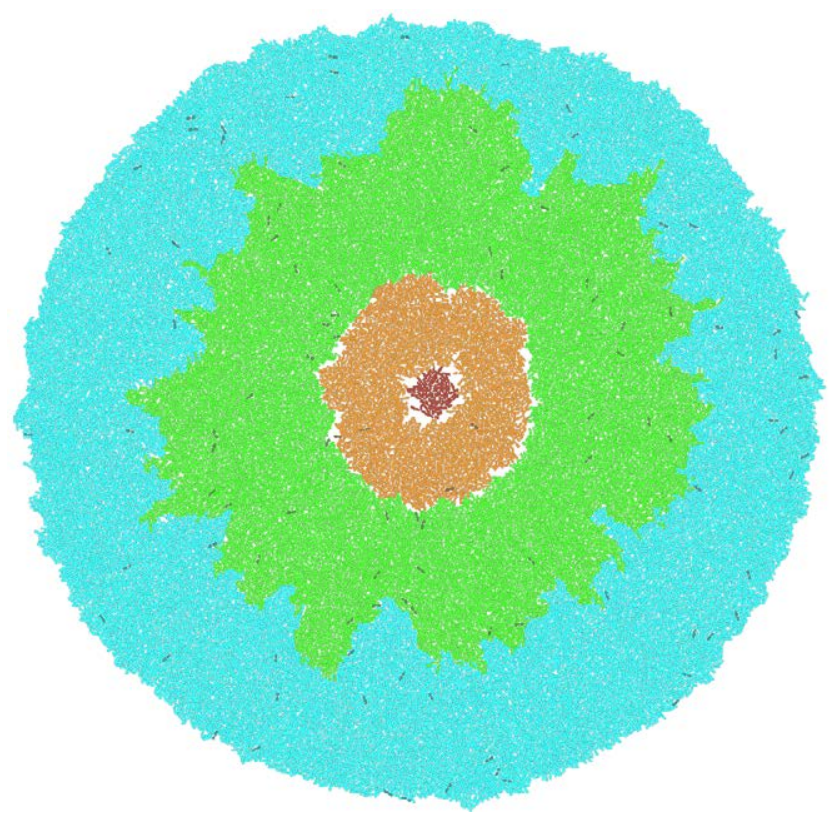

Figure 4.20: Four-level multicellular bullseye pattern. By generalizing the idea presented in the previously shown three-level bullseye pattern, the number of zones can now scale. Source code for this simulation is present in Appendix B.5.

The presented circuits are heavily based on the conjugation and signals functionalities now present in gro. Of course, large colonies are also necessary to be capable of observing the patterns emerge in time. 


\section{Spatial oscillator}

Another spatio-temporal pattern circuit that was designed and simulated is based on an oscillator in which cell growth induces a ripple pattern. The desired outcome for the design is shown in Figure 4.21.
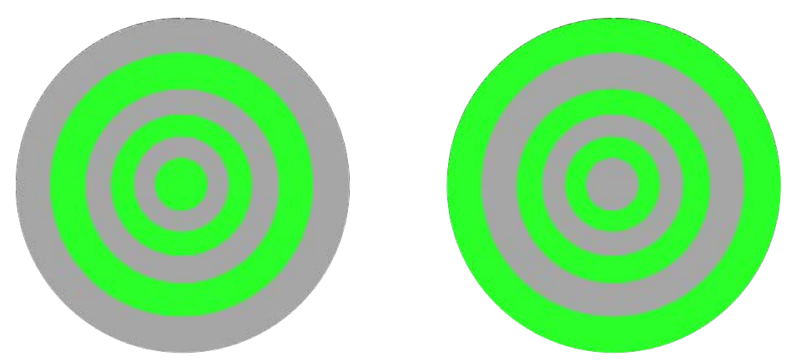

Expected result for the spatial oscillators

Figure 4.21: Expected results for spatial oscillator pattern circuits. The left panel shows a pattern in which the central part initiates the pattern generation and the ripple pattern builds as cells grow outwards. The right panel shows a variant that represses the central bacteria and coordinates oscillations to give rise to the pattern.

By adapting the idea of using oscillations for generating a spatio-temporal pattern RSLY12, KK17, and implementing further conditions for its operation specific designs for a genetic circuit were reached. The circuit is based on coordinating the start in oscillations with an environmental signal such as IPTG. Given this coordination, all bacteria exiting the repression zone marked by the signal will go through the oscillating phases at the very similar times, accounting for the generation of rings. The distance separating each ring is given by the period of oscillation and the growth rate of bacteria. The circuit design is shown in Figure 4.22, 

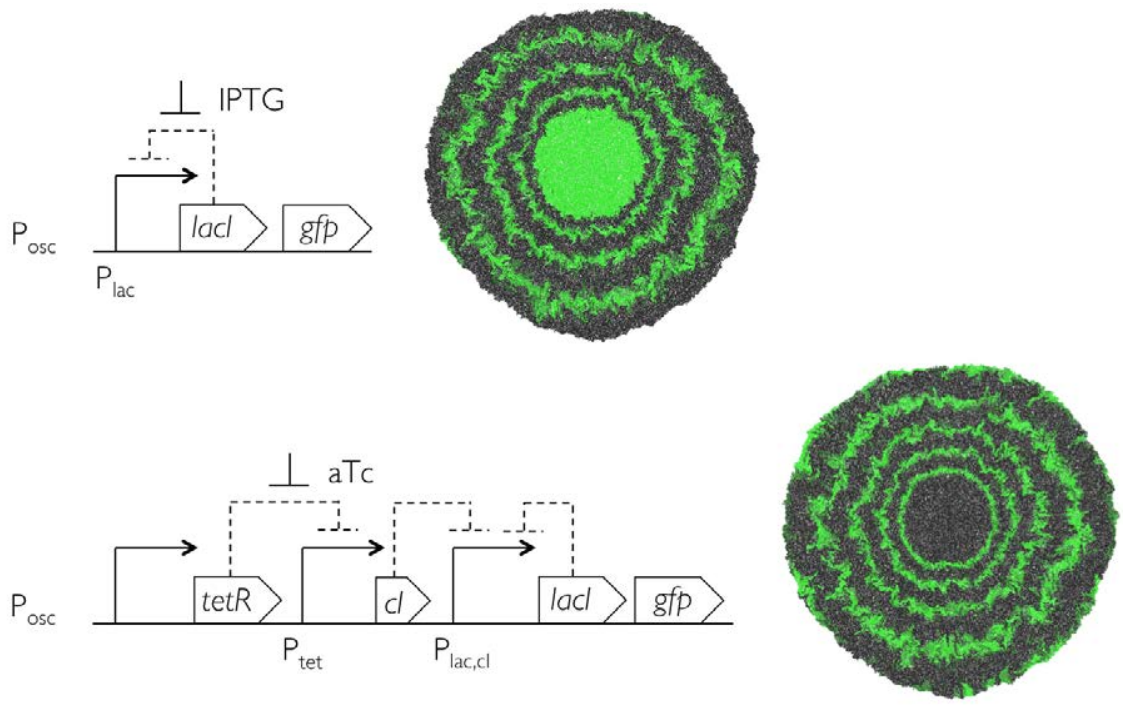

Figure 4.22: Spatial oscillators. Two circuits implement this design: The top circuit is a negative feedback implemented by lacI that reports its expression through GFP. In this case, the high concentration zone of IPTG is reported, as it prevents repression from lacI. The bottom circuit adds a control switch that represses lacI and GFP where aTc is in high concentration. The ripple pattern emerges due to the oscillations beginning at similar times and bacterial growth moving bacteria outwards. The further a GFP expressing ring is from the colony center, the larger its width. In these simulations, no variability is considered and very fast degradation times are set. Details can be found in Appendices B.6 and B.7

The time for lacI to repress itself and $G F P$ is a key parameter in this design, as it produces dark intervals between $G F P$ expressing rings. Changes in this parameter and $G F P$ degradation have an effect on the width of $G F P$ rings. Conversely, GFP expression time translates to the width between GFP expressing rings.

Noise and variability in the expression and degradation times make the pattern fuzzy but still present, as shown in Figure 4.23 (source code with details found in Appendices B.8 and B.9. 

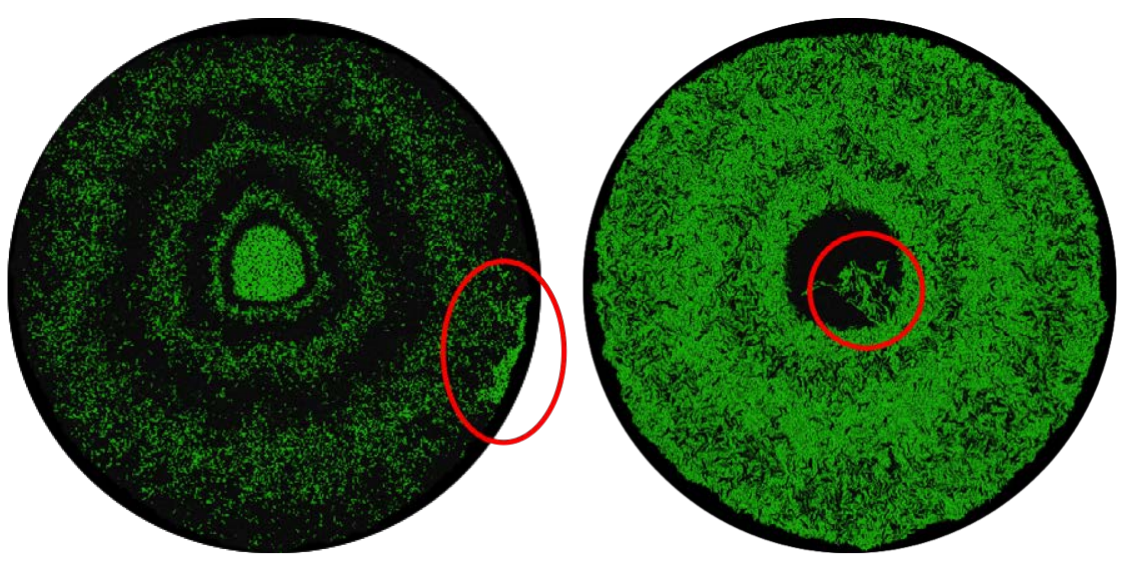

Figure 4.23: Spatial oscillators with noise and variability. Left: no switch is used. The pattern is not as visible as in the previous case, however it is still observable and clear. Right: the pattern is inverted since the repression switch is present. Rings forming the ripple pattern are now the dark ones, but are now less distinguishable. Noise is also visible in the colonies and marked in red.

The designs presented here can be applied to any genetic oscillator MBRP11, $\mathrm{SCB}^{+} 08$, EL00, including those following the Goodwin model GAJ13, RVMR99, Goo65, since mechanical interaction between bacteria is what drives pattern formation for these patterns. No communication is needed between bacteria. Only the starting condition in the center must be applied to the whole colony.

\section{Sun pattern}

A final spatio-temporal pattern will be presented: a circular core with "rays" extending beyond it. It could be classified as a fluid-spreading or Dense-BranchingMorphology type pattern $\left[\mathrm{TKM}^{+} 09, \mathrm{WRI}^{+} 98\right.$. A depiction of the expected outcome is shown in Figure 4.24

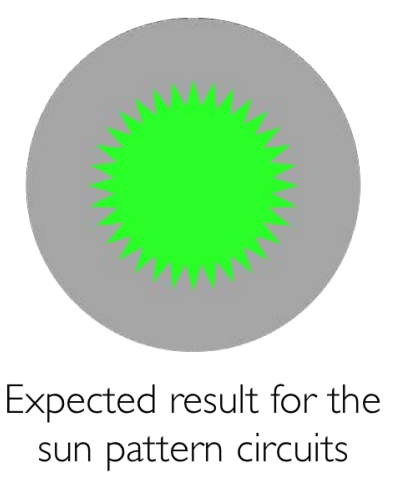

Figure 4.24: Expected results for sun pattern circuits. The pattern should concentrate glowing bacteria in the colony center and multiple filaments of glowing bacteria stemming outwards from this central core. 
It was simulated in gro with two different methods:

- The first method consisted in using nutrient consumption to restrict cell growth. Using a gradient distribution of nutrients, bacteria approaching an area of low nutrient concentration start to grow in filaments. The phenomenon can be explained because of the nutrient sufficient areas being reached by a few bacteria due to mechanical impulse. Figure 4.25 shows the simulation result. The reader can remark similarity to nutrient profile Gradient/High shown in Figure 4.16

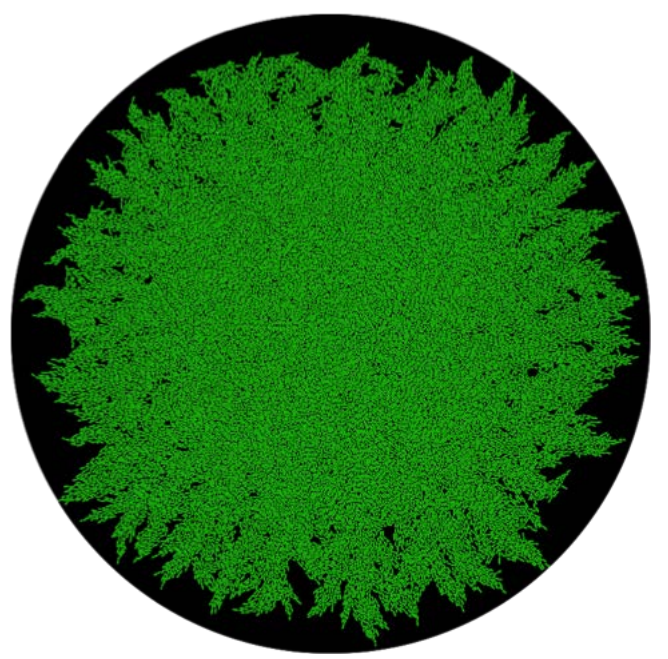

Figure 4.25: Sun pattern generated with nutrient consumption. Outward areas with lower amount of nutrients restrict cell growth, making it only possible for bacteria being pushed outwards to reach zones where nutrients have not been depleted yet. In turn, these bacteria push new ones further, giving rise to the pattern spikes.

- The other method for generating the pattern was through a synthetic circuit. A central circular GFP-expressing core is delimited by an IPTG zone. In this zone, bacteria classified as "emitters" contain a plasmid $\left(P_{E}\right)$ that induces AHL production and conjugation of itself. Another type of bacteria, "receivers", containing a second plasmid $\left(P_{R}\right)$. These bacteria absorb AHL re-emitting it, expressing $G F P$, and conjugating itself when the concentration of AHL is sufficient. The effect is a relay system on AHL in which the rays are produced by bacteria that read enough of the AHL trail outside the center core and have the capacity to pass the relay by conjugating. Design and simulation of the circuit are shown in Figure 4.26 

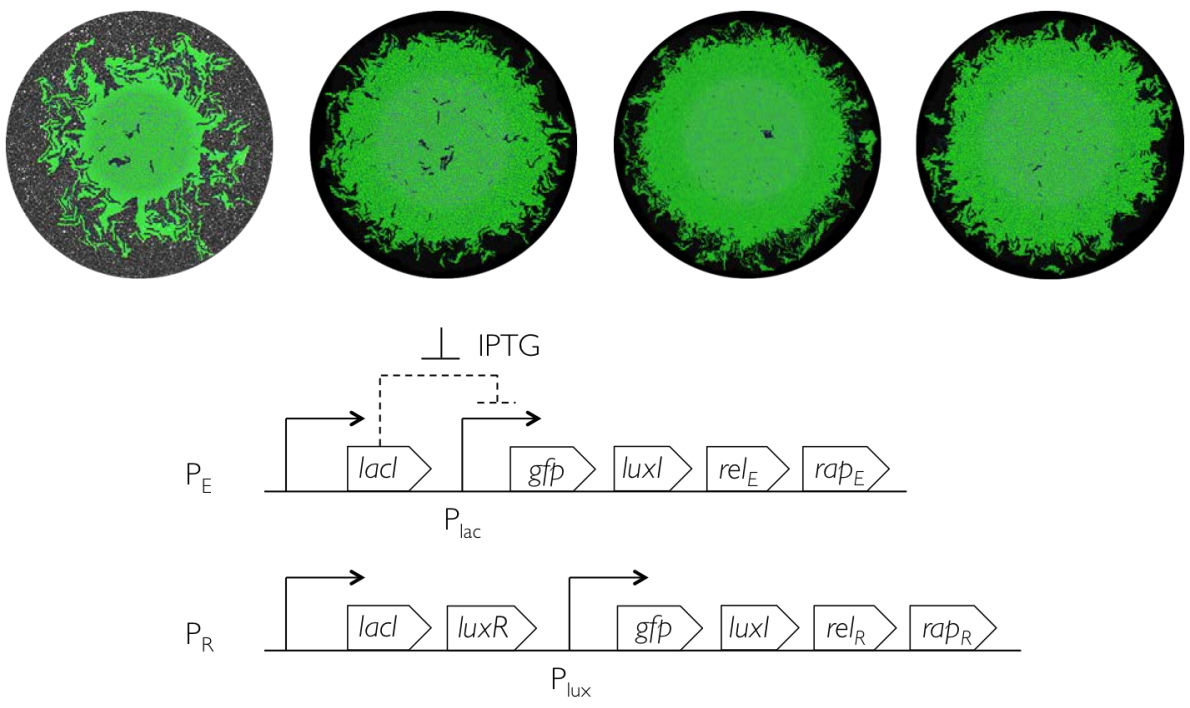

Figure 4.26: Sun pattern generated with a synthetic circuit. Top: simulations resulting in the sun pattern. Each run has a different ratio of Emitter:Receiver bacteria. The ratios are, respectively, from left to right: 1:10, 1:5, 1:2 and 1:1. Bottom: emitter and receiver plasmids. The inner core section of the pattern is colored by $P_{E}$, while the outer spikes are due to $P_{R}$ expressing GFP. $P_{E}$ can only conjugate in the zone where IPTG is present, while $P_{R}$ only conjugates when AHL is detected.

Although both designs produce similar patterns, some differences are noted:

1. Generation with nutrients results in a continuous mass of bacteria, where the colony makes up the pattern shape. However, generation with the synthetic circuit bears discontinuities, as not all receiver bacteria are close to each other.

2. Spikes in the nutrient generated pattern are thicker and smoother than in the synthetic one. This is explained because of the difference in how the pattern is generated. In the first case, it is mechanical forces. However, for the synthetic circuit, it depends on the AHL trail and bacterial conjugation.

3. In the synthetic circuit, the inner core has a defined circular shape, while for the nutrient generated one, it is unclear where the boundary starts. This is due to the IPTG cue present in the synthetic circuit being sharper than a gradient concentration of nutrients.

The sources for both the nutrient and synthetic version of the simulations can be found in Appendices B.10 and B.11 respectively. 


\subsubsection{Temporal patterns}

A temporal pattern circuit was also simulated with gro. Although the system is better suited for simulating circuits that exhibit a spatial component, it can also be used for working without it. Such an example has been implemented and run.

\section{Autonomous bioreactor - Sequential circuit}

The idea behind this circuit is to reproduce a sequential machine in which tasks are completed in order one after another. Each task has a duration. Once this time is over, the next task must be executed. The circuit can be deemed an "Autonomous bioreactor" in the sense that it requires no external control to switch from one task to the next, and bacteria are well-mixed. An idea of the desired outcome is shown in Figure 4.27 .

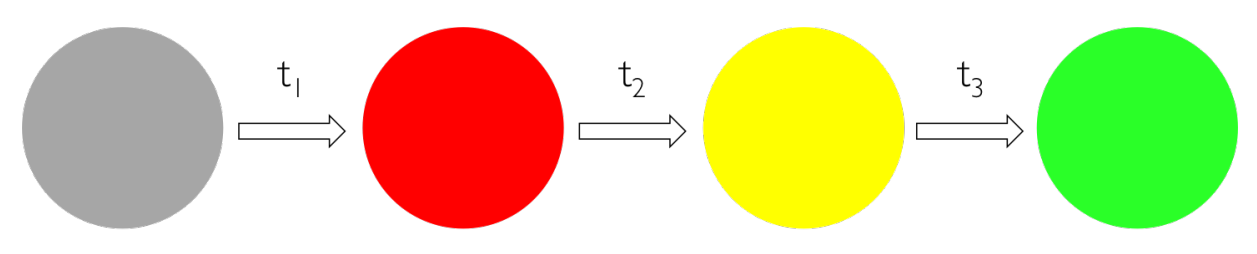

Expected result for the autonomous bioreactor circuit

Figure 4.27: Expected sequence for the autonomous bioreactor. Being a temporal pattern, bacteria in the colony should display only a single color at a given time, marking specific phases in the reaction sequence. The design is expected to produce $R F P$ first, $Y F P$ next and $G F P$ in the end.

The implementation of this circuit is distributed, as each task or step of the sequence is encoded in a different plasmid. Specifically, each task has been replaced with a reporting protein as a placeholder (RFP, YFP and GFP, in that order). A plasmid is responsible for the execution of a single task, and no conjugation is present in the design. This yields a division of labor in which any bacterium carrying a plasmid is responsible only for the task it encodes.

The circuit is based on a signaling system in which a signal emitted by any plasmid encoding a task initiates the next task in the sequence. This same signal triggers elimination of the plasmid that is executing the current task and emitting the signal. Therefore, this process ceases the current task in the form of a negative feedback with delays. The design is shown in Figure 4.28. Simulations for were also run. Results are shown in Figure 4.29. 

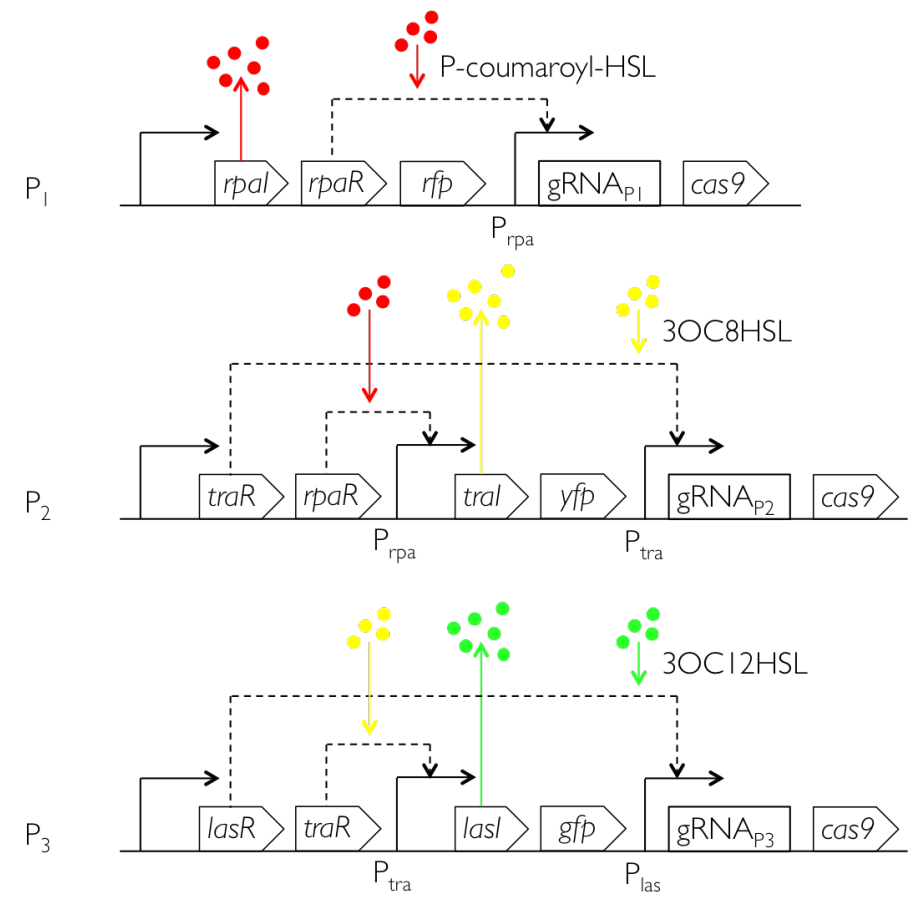

Figure 4.28: Design of the Autonomous Bioreactor. A sequence of three tasks is designed, each task being encoded by a plasmid in the form of a fluorescent protein. Communication is based on signals that direct the start/end of a task in the sequence and induce plasmid loss with a CRISPR/Cas9 system. The circuit is scalable: by reproducing the design of $P_{2}$ or $P_{3}$, it is possible to extend the sequence arbitrarily.

Since this is a distributed circuit and no cell executes more than a single task, an arbitrarily long sequence may be constructed. The design of this circuit is scalable. This scalability is however restrained by orthogonality of the involved signaling communication mechanisms. Recent work has been published on this matter [SH16] and provides a set of systems that prove to be adequate for this design. 

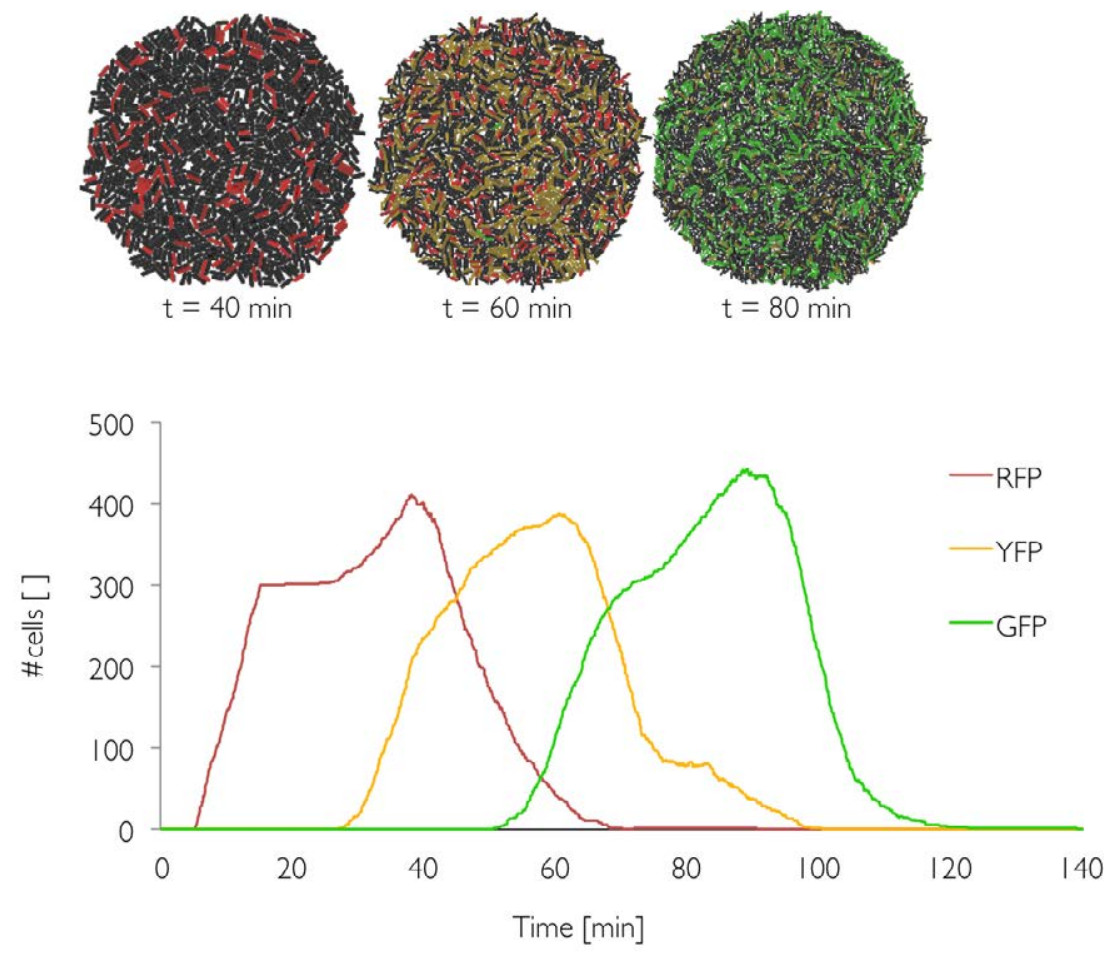

Figure 4.29: Simulation of the Autonomous Bioreactor. Top: all three tasks are depicted at the time of their maximal expression. The circuit dynamics produces pulses that represent execution of each task. There is a difference of about 20 minutes between maximal expression of each fluorescent protein. The sequence to be executed is $R F P$, followed by $Y F P$ and finally GFP. Bottom: the number of cells expressing each fluorescent protein (task) is recorded over time. Pulses are observed that have a duration of about $60+/-10$ minutes and produce peaks at 37 minutes $(R F P), 61$ minutes $(Y F P)$ and 89 minutes $(G F P)$. A pulse temporal pattern is observed as the outcome of the circuit. Reprinted with permission from Gutiérrez et al GGGPdP $^{+} 17$. Copyright 2017 American Chemical Society.

The source code for this circuit can be found in Appendix B.12.

\subsection{Discussion}

gro has undergone major changes in the course of this work. All of the implemented work for the platform can be found at https://github.com/liaupm/GRO-LIA Also, the platform was tested by simulating novel multicellular circuits. Many assumptions and decisions were taken for each of the functionalities implemented. In this section, a discussion on consequences and limitations will be provided for the choices that were made and why other alternatives were discarded. 


\subsubsection{CellEngine}

The acceleration of gro translated into simulating a larger amount of bacteria. This acceleration has not interfered with simulation accuracy. The major bottleneck was found to be located in the physics management of bacteria. The problem could be tackled through several different paths:

- Parallelize the shoving algorithm for cell physics

- Tweak parameters of the existing algorithm

- Design a new algorithm for cell shoving

Parallelization was initially attempted by using OpenMP Int. However, most possible parallelization sites were few and difficult to find, since most of the resources such as the population set (a container of all bacteria in the simulation) or the environment itself (called "world") are shared and must be sequentially accessed. A parallel version of gro was implemented, however, no speedup was observed. A possible explanation for this event is that threads in the OS and in gro itself already handle parallel execution. Another possible explanation is that whatever gain is produced by the parallelization is lost in communication overhead.

The second alternative, changing parameters for the existing shoving algorithm resulted in an acceptable speedup. Specifically, the amount of collision detection-response iterations was the parameter being altered. This change sped up the software by a time proportional to the decrease in number of iterations. However, the reduced computing time came at a great cost: physics was not accurate, meaning cells exhibited many overlap and also cell density in the colony was much lower.

Finally, designing a new algorithm and its development was the chosen path that gave rise to the observable speedup used presently in gro. Validation of CellEngine is shown in Figure 4.9. Parameters used to assess the correct functioning of CellEgine included cell orientation and colony density [VCHT08, $\mathrm{CJC}^{+} 07$. It should be however noted that despite offering a great increase in speed and no accuracy loss, two directives present in the original version of gro had to be disabled: chemostat mode and chemotaxis. The main reason for this was that chemostat mode simulated a liquid flow with solid barriers preventing cell growth beyond those limits. The shoving algorithm of CellEngine cannot handle these constraints in its current implementation. Chemotaxis was also removed since cell movement also interferes with the ring algorithm for shoving.

\subsubsection{CellPro and ProSpec}

A major change in gene expression modelling occured in gro as originally it involved a set of variables to model the various stages of gene expression. All of the values represent concentrations, thresholds, several kinds of rates (transcription, translation, degradation, etc.) and times. The decision was made to first, simplify all of the steps into two events: 1) Transcription factors binding to the promoter and initiation of the expression process. 2) Unify all of the 
time delays into a few of them that direct the protein dynamics (activation and degradation).

The operation over these two events yield a binary model in which proteins are at the center of the simplification. This first shift in paradigm requires a binary model, meaning all model operations should be based on binary inputs with time delays. From an implementation standpoint, this implies that by working at a binary level, CellPro operations are much faster, as genetic elements being represented are seen as bits and can take advantage of low-level bit operations. Another consequence is that the binary philosophy is extended to the specification language, therefore, interpreting many of the biological elements as binary ones, such as logic gates in place of promoters, for instance.

This simplification also conveys limitations such as the impossibility of simulating impedance mismatch MW05. As the circuits are now described in binary terms, all " $0 \mathrm{~s}$ " and "1s" are the same. This restricts the realism of simulated genetic circuits to a certain degree, since most of the time they are not readily combinable. Of course, in an analogical framework, representing impedance mismatch is possible, however the complexity for describing circuits and its parametrization is a very tedious endeavor.

Variability and noise are more elements upon which several possibilities were considered. Since the work environment is a binary one, a source of error should be modeled as a complementary value. For instance, a malfunctioning promoter, can be expressed as a logic gate that outputs a fixed value (permanent malfunction) or an erroneous output. A decision to make was which kinds of malfunction should be included. Such possibilities were always linked to temporal duration: permanent or transient. Biological similarity pointed towards an implementation of permanent failure, as it could represent mutations avoiding the correct expression of the protein or similar proteins misfiring the whole process. A transient malfunction was left out because no viable link was found to noise observed in genetic circuits.

As for ProSpec, the specification language is aligned with CellPro and aims to resemble genetic structures. It is constructed as an extension of CCL Kla04 to complement the already existing guarded commands with protein-oriented programming GGGPdP ${ }^{+} 17$. To allow for continuity of simulations already implemented in the original version of gro and as a way of extending them, ProSpec was built atop CCL. An alternative could have been to completely remove CCL and replace it with ProSpec or another specification language. There are at least two limitations stemming from keeping CCL as a component of the system: 1) Parsing the a specification imposes a great burden on the system and greatly slows it down. 2) CCL restricts certain grammatical constraints, imposing a given structure on the data extracted from the specification. This underlying relationship between the components still enables a rich set of instructions to be included (even with a combination of guarded commands and protein-oriented specification). However, it is fragile in the sense that extending both specification languages even further increases in difficulty and constraints. 


\subsubsection{Bacterial Conjugation}

For bacterial conjugation, implementation of the transference process was straightforward, since only the neighborhood of a bacterium was needed. However, control of this transference process to accurately simulate bacterial conjugation implied other questions such as: Does it occur based on frequency or density of neighbors? How is this occurrence of conjugation translated to an individual case? Is there data for this, or is all data for populations? Many of these questions do not have an answer yet, as conjugation is not fully understood at the individual cell level.

As described in Algorithm 4.1, a mixed version of conjugation was implemented to account for both possibilities - frequency and density approaches of conjugation. The critical neighborhood size $N_{c}$ parameter can be tuned so that in case it is discovered that the process is fully frequency based, the density parameters may disappear. Conversely, a configuration setup can be arranged to make conjugation purely density-based. This setup would rely entirely on the actual number of neighbors $N_{b}$ and the critical neighborhood size $N_{c}$, eliminating the frequency $(\gamma)$ and generation time $(G)$ dependence.

In the spirit of providing realism and additional functionalities concerning bacterial conjugation, a surface entry exclusion mechanism was also implemented. However, it is possible that exclusion of a plasmid can occur after it has entered the bacterium through conjugation or some other means. Therefore, the loss of a plasmid is another implemented operation that covers one more possibility for plasmid disappearance or exclusion.

Justification of a correct simulation of bacterial conjugation was done against population data, describing the dynamics of conjugation in terms of donor (D), recipient $(\mathrm{R})$ and transconjugant $(\mathrm{T})$ bacteria amounts. The input experimental data was produced by Fernando de la Cruz's lab in Santander $\mathrm{dCRC}^{+} 12$, $\mathrm{FLDR}^{+} 14$.

\subsubsection{CellSignals}

Extending CellSignals was straightforward in the analog case, as the diffusion method is the same (Finite Element Method). New functionalities were based on the capability of passing values as input and executing already possible functions such as emission or absorption in different ways. However, a motivation for digital signals proved to be a challenge, as a diffusion method had to be devised and more importantly, modeled to align with gro.

The digital variant of signals was based on a cellular automaton model because of its discrete nature both spatially and in terms of the signal values. In fact, the model that was used is a block automaton. In such a model, each grid cell has a container for multiple signal units. This set can be seen as a smaller, second grid within each cell of the global signal matrix. The smaller grid allocates particles of digital signal at given locations. Diffusion is implemented as a mechanism in which the smaller grid cells exchange signal particles with a given probability, and according to their location. The dynamics is that of a random 
walk algorithm in the movement of particles.

Digital signals were originally thought as a light approximation for the analog type of signals. Although this philosophy is valid and loosely simplifies analog signal diffusion, its real usefulness might be directed towards tracking individual signal particles. In making the grid sufficiently dense, despite the large computational cost it would imply, a finer control of particles would be attainable.

The vision for this type of model will be mentioned in Chapter 5 .

\subsubsection{CellNutrient}

Consumption of nutrients is a widely characterized process and has well known models to simulate it. The model most aligned with the gro framework is the Monod consumption-growth model. The reason for this choice being that it is based on nutrient quantity and due to the model simplicity [SH11. Therefore, this was the model implemented in the framework. However, other possible models were alternative choices, such as Blackman kinetics DFW73, Stannard, Gompertz, logistic, and other models [ZJRVR90. All of these models are still open to be included in gro.

\subsubsection{Multicellular circuits}

The simulation of multicellular circuits atop the new gro version demonstrates the simulator potential for prototyping multicellular genetic circuits using conjugation. The whole array of novel functionalities have been used in the proposed circuits simulation. This confirms that all of them are useful, and in the case of intercellular communication, necessary, as no local mechanism was present in the platform previously. Also, the results produced by the simulator have been enriched in that communication mechanisms can now be combined and more complex circuits specified and simulated.

Parameters driving the specific implementation of each pattern can be summarized as follows:

- Bullseye patterns:

- 2-level pattern: Sensitivity to IPTG thresholds and activation and degradation times translate to the size of the non-glowing gap.

- 3-level pattern: Bacteria ratio is important to determining initial distribution of the cells. Bacterial generation time is also a key parameter, as an equilibrium must be achieved with cells being killed. Sensitivity to the environmental signal and delays until death is a key parameter as it regulates the speed at which a specific zone clears bacteria that do not belong there.

- 4-level pattern: Extension of the 3-level case.

- Spatial oscillator: Activation and degradation times for $G F P$ are the key parameters along with sensitivity to IPTG/aTc. Activation and degradation times establish ring width of the ripple pattern, while the sensitivity 
to environmental signals dictates how large the center core is. When the environmental signal is no longer detected, oscillations are initiated. Less important parameters include noise and variability, since they slightly disorganize the pattern, although it is still generated.

- Sun pattern:

- Nutrient consumption: The only parameter affecting the obtained pattern in this case is the nutrient amount (although a gradient distribution is used). The distance between the colony center and the filament start decreases proportionally to the initial available nutrient amount.

- Synthetic circuit: IPTG sensitivity defines the inner core size, whereas the filament continuity and width depend strongly on the sensitivity to $A H L_{(\operatorname{lux})}$. The lower the sensitivity, the more discontinous and thin the filaments are.

- Autonomous bioreactor: Activation and degradation times, along with sensitivity to QS signals drive the speed at which transitions in the system are undergone. Plasmid destruction triggers the end of a stage, in turn forcing degradation of the protein being produced in that stage.

These designs were obtained by establishing a desired outcome and iterating on their specification whenever flaws were encountered. This follows the exact same goal for which the platform was constructed: fast prototyping of multicellular circuits. As the platform toolkit was fully tested in the design simulation, an advance on the previous circuits that could be simulated is shown. Feedbacks, Feed-forward loops, noise are all complex circuits that can be simulated and are shown in this document with the spatial oscillator, for example. Binary proteins prove to be a robust representation scheme as they are capable of reproducing these effects. Naturally, as more capabilities are added to the toolkit, even more complex circuits will be prototyped. 


\section{Chapter 5}

\section{Conclusions and future work}

\subsection{Conclusions}

gro and the developed modules to complement it are open-source. The LIA group and myself hope that many research groups collaborate with us in its future development.

In this work, two original contributions have been presented:

- A new version of the gro platform has been implemented that is faster and offers new tools for simulating multicellular circuits.

- New designs involving bacterial conjugation were developed and simulated with the new version of gro.

These contributions, along with an analysis of the State of the art presented in Chapter 2 answer the questions posed in Chapter 3 . Conclusions relating to each of the problems that have been solved in this work are now stated.

\section{The new gro platform}

A new version of gro has been developed which now presents a larger toolkit and improves upon its previous version in terms of speed and capabilities. gro can now easily simulate $10^{5}$ bacteria in a matter of minutes (instead of a week). This newly-acquired speed of the simulator places it as a very fast prototyping tool for a wide range of systems and synthetic biology designs. Additionally, gro now provides built-in genetic specification and dynamics, nutrient consumption, improved environmental signal capabilities and bacterial conjugation. Realism of the simulations produced by the platform has increased as high cell counts are now attainable and can make use of the new features. Outcomes of these simulations accurately shed light on the behavior of multicellular circuits, fulfilling their purpose in prototyping for early assessment of circuit design. This makes it a tool that is useful for a broader audience than its previous version. Multicellular systems biology, microbial evolutionary biology and bacterial epidemiology are examples of other fields that could benefit from a new platform 
like the one presented in this document.

Specifying a circuit for the platform to simulate is now less complex, since most of the calculations involved in gene expression dynamics have been simplified and are done internally. Previously, many variables such as expression and degradation rates, concentration and time counters, promoter conditions, and many more needed to be controlled for implementing these dynamics. A novel programming paradigm directed by proteins is proposed as the alternative method for writing specifications in gro. This paradigm summarizes and greatly simplifies the specification of a circuit as the underlying gene expression model requires only a few parameters to execute. Despite this, a user who does not wish to use the new specification paradigm may still use the original guarded command based language. This means that most simulations implemented for the preceding version of gro still work when they are simulated with the current version. ProSpec resembles SBOL in terms of genetic circuit structural description. SBOL represents an organized, but static view of genetic circuits. However, parameters associated with ProSpec in addition to its link with CellPro, make the specification into a dynamic one. Also, the underlying model based on digital proteins provides a framework where describing genetic circuits is simplified and reminiscent of digital logic ones. The cost for losing precision in analog concentrations of the proteins, mainly associated to impedance mismatch, is compensated by the boost in speed, simplicity in the description of circuits and simulation of a wide range of multicellular circuits.

The extension of gro has produced a system architecture and methodology that leave the path open to more improvements and advancements. External modules implementing functionalities in the system are an optimal solution, as these modules can be reused at a later time by a different system, and their role is well defined. However, care should be taken in future additional developments of gro, as the system is outgrowing its original goal: to simulate multicellular behaviors in microcolonies. Deep structural changes would be required to make the platform further, openly and more easily extensible. Specifically, CCL imposes a rigid structure upon the gro architecture, as it restricts the order in reading data for the specifications and setting up the environment. This makes adding new parameters and organizing the new modules that use them a difficult task. Guidelines to address this problem will be discussed in Section 5.2 .

\section{Novel biocircuits}

A large array of designs have been prototyped with gro. Although its main strength lies in the simulation of spatio-temporal patterns, gro is also a valid tool for studying other kinds of models. Examples include microbial population interaction, information processing multicellular circuits or developmental microbiology. Gutiérrez et al. [GGGPdP ${ }^{+} 17$ ] presented two examples in systems biology: a study on plasmid propagation and simulations on microbial population interactions. This paper also showed an elementary information processing circuit in the form of a YES logic gate. Most of these designs were selected as a potential validation for each of the novel features that were included into the platform. 
Novel biocircuits have been proposed in this work. These designs strongly rely on the new capabilities added to gro. Intercellular communication in the form of bacterial conjugation and environmental signals, along with the new protein-oriented programming paradigm and built-in protein expression mechanisms played a major role in the design of these circuits. Another goal was achieved, as novel multicellular biocircuits using conjugation and proposed in the PLASWIRES project that have benefited from the new platform and have been successfully simulated.

Combination of intercellular communication mechanisms is now possible in gro simulations. The spatial patterns presented in Section 4.3.1 are examples of designs that use both conjugation and environmental signals. gro provides an environment in which new designs can be prototyped and easily tinkered with.

\subsection{Future work}

Future (and current) research and development directions stemming from this work are proposed here. These proposals are directly related to gro and its capabilities, although they also would bear an impact on the state of the art concerning AbM microbial simulators.

Further extension of gro requires a careful revision of its original components. A recommendation in this direction is the replacement of CCL and a reorganization of the gro source code to pipeline the data input process and its use. Currently, gro is forced into an execution sequence in which key variables needed to set up the simulation environment cannot receive their value when required. Currently, LIA is working in this direction.

Automation in specification generation will help eliminate human error introduced when designing a circuit. A web-based graphical interface using Blockly [Goo and following the Bioblocks [GIPRP17] implementation is currently being developed by LIA to this end. In parallel to this interface, a client-server architecture is being implemented to execute gro through a web browser, eliminating the need for software installation. Another interesting direction related to these advancements is the import/export and use of SBOL $\mathrm{RBC}^{+} 16$ and/or SBML $\left[\mathrm{HFS}^{+} 03\right]$ for gro specifications.

Concerning gro functionalities, several ongoing research niches remain open: CRISPR/Cas9 system for gene editing, exploring other nutrient consumption models such as Blackman Kinetics, investigating the use of CellSignals for modeling phage propagation, replacement for finite element method in signal diffusion, further research on the shoving algorithm for CellEngine to enable chemostat mode and chemotaxis again.

ProSpec, being a scalable specification language layer should also be extended along with gro to make it capable of describing gene editing (CRISPR/Cas9 system), RNA signals or bacteriophage propagation to name a few extensions. 


\section{Bibliography}

[ABKW06] Ernesto Andrianantoandro, Subhayu Basu, David K Karig, and Ron Weiss. Synthetic biology: new engineering rules for an emerging discipline. Molecular Systems Biology, 2(1), 2006.

[Alo07] Uri Alon. Network motifs: theory and experimental approaches. Nature Reviews Genetics, 8(6):450-461, 2007.

[Amo14] Martyn Amos. Population-based microbial computing: a third wave of synthetic biology? International Journal of General Systems, 43(7):770-782, 2014.

[App85] Andrew W Appel. An efficient program for many-body simulation. SIAM J. Sci. Comput., 6(1):85-103, 1985.

[Ark08] Adam Arkin. Setting the standard in synthetic biology. Nature biotechnology, 26(7):771-773, 2008.

[ASMN03] Mariette R Atkinson, Michael A Savageau, Jesse T Myers, and Alexander J Ninfa. Development of genetic circuitry exhibiting toggle switch or oscillatory behavior in escherichia coli. Cell, 113(5):597-607, 2003.

[AVA07] J Christopher Anderson, Christopher A Voigt, and Adam P Arkin. Environmental signal integration by a modular and gate. Molecular Systems Biology, 3(1):133, 2007.

$\left[\mathrm{BGC}^{+}\right.$05] Subhayu Basu, Yoram Gerchman, Cynthia H Collins, Frances H Arnold, and Ron Weiss. A synthetic multicellular system for programmed pattern formation. Nature, 434(7037):1130-1134, 2005.

[BM17] Hasan Baig and Jan Madsen. D-vasim: an interactive virtual laboratory environment for the simulation and analysis of genetic circuits. Bioinformatics, 33(2):297-299, 2017.

[Boe88] Barry W. Boehm. A spiral model of software development and enhancement. Computer, 21(5):61-72, 1988.

[BS00] Attila Becskei and Luis Serrano. Engineering stability in gene networks by autoregulation. Nature, 405(6786):590-593, 2000.

[BS05] Steven A Benner and A Michael Sismour. Synthetic biology. Nature Reviews Genetics, 6(7):533-543, 2005. 
[BSE12] Jerome Bonnet, Pakpoom Subsoontorn, and Drew Endy. Rewritable digital data storage in live cells via engineered control of recombination directionality. Proc. Natl. Acad. Sci. U. S. A., 109(23):8884-8889, 2012.

$\left[\mathrm{BSO}^{+} 08\right]$ Frederick K Balagaddé, Hao Song, Jun Ozaki, Cynthia H Collins, Matthew Barnet, Frances H Arnold, Stephen R Quake, and Lingchong You. A synthetic escherichia coli predator-prey ecosystem. Mol. Syst. Biol., 4(1):187, 2008.

[BYA08] Katie Brenner, Lingchong You, and Frances H Arnold. Engineering microbial consortia: a new frontier in synthetic biology. Trends in biotechnology, 26(9):483-489, 2008.

$\left[\mathrm{C}^{+} 70\right]$ Francis Crick et al. Central dogma of molecular biology. Nature, 227(5258):561-563, 1970

$\left[\mathrm{CFCC}^{+}\right.$03] Carlos Canchaya, Ghislain Fournous, Sandra ChibaniChennoufi, Marie-Lise Dillmann, and Harald Brüssow. Phage as agents of lateral gene transfer. Current Opinion in Microbiology, 6(4):417-424, 2003.

[CGK12] George M Church, Yuan Gao, and Sriram Kosuri. Nextgeneration digital information storage in DNA. Science, 337(6102):1628-1628, 2012.

[Chr04] Peter J Christie. Type iv secretion: the agrobacterium virb/d4 and related conjugation systems. Biochimica et Biophysica Acta (BBA)-Molecular Cell Research, 1694(1):219-234, 2004.

$\left[\mathrm{CJC}^{+}\right.$07] HoJung Cho, Henrik Jönsson, Kyle Campbell, Pontus Melke, Joshua W. Williams, Bruno Jedynak, Ann M. Stevens, Alex Groisman, and Andre Levchenko. Self-organization in highdensity bacterial colonies: Efficient crowd control. PLoS Biology, 5(11):2614-2623, 2007.

[CLM11] Po-Ning Chen, Hsuan-Yin Lin, and Stefan M Moser. Ultrasmall block-codes for binary discrete memoryless channels. In Proceedings of the IEEE Information Theory Workshop, pages 175-179. IEEE, 2011.

[Col03] Nick Collier. Repast: An extensible framework for agent simulation. The University of Chicago's Social Science Research, 36:2003, 2003.

[Cri58] Francis HC Crick. On protein synthesis. In Symp Soc Exp Biol, volume 12, pages 138-163, 1958.

$\left[\mathrm{CRRP}^{+} 15\right]$ Elena Cabezón, Jorge Ripoll-Rozada, Alejandro Peña, Fernando de la Cruz, and Ignacio Arechaga. Towards an integrated model of bacterial conjugation. FEMS microbiology reviews, 39(1):81-95, 2015. 
[CW54] Francis HC Crick and James D Watson. The complementary structure of deoxyribonucleic acid. In Proceedings of the Royal Society of London A: Mathematical, Physical and Engineering Sciences, volume 223, pages 80-96. The Royal Society, 1954.

[dCRC $\left.^{+} 12\right]$ Irene del Campo, Raúl Ruiz, Ana Cuevas, Carlos Revilla, Luis Vielva, and Fernando de la Cruz. Determination of conjugation rates on solid surfaces. Plasmid, 67(2):174-82, March 2012.

[DFW73] JN Dabes, RK Finn, and CR Wilke. Equations of substratelimited growth: The case for blackman kinetics. Biotechnology and bioengineering, 15(6):1159-1177, 1973.

[DlCD00] Fernando De la Cruz and Julian Davies. Horizontal gene transfer and the origin of species: lessons from bacteria. Trends in Microbiology, 8(3):128-133, 2000.

[DR82] Jim Douglas, Jr and Thomas F Russell. Numerical methods for convection-dominated diffusion problems based on combining the method of characteristics with finite element or finite difference procedures. SIAM J. Numer. Anal., 19(5):871-885, 1982.

[DTD $\left.{ }^{+} 14\right]$ Laurent Drazek, Maud Tournoud, Frédéric Derepas, Maryse Guicherd, Pierre Mahé, Frédéric Pinston, Jean-Baptiste Veyrieras, and Sonia Chatellier. Three-dimensional characterization of bacterial microcolonies on solid agar-based culture media. $J$. Microbiol. Methods, 109:149-156, dec 2014.

[EL00] M B Elowitz and S Leibler. A synthetic oscillatory network of transcriptional regulators. Nature, 403(6767):335-338, 2000.

[End05] Drew Endy. Foundations for engineering biology. Nature, 438(7067):449-453, 2005.

[Eri04] Christer Ericson. Real-time collision detection. CRC Press, 2004.

[EZ17] Yaniv Erlich and Dina Zielinski. Dna fountain enables a robust and efficient storage architecture. Science, 355(6328):950-954, 2017.

[FHMW13] F D C Farrell, O Hallatschek, D. Marenduzzo, and B. Waclaw. Mechanically driven growth of quasi-two-dimensional microbial colonies. Phys. Rev. Lett., 111:1-8, 2013.

[FLDR $\left.{ }^{+} 14\right]$ Raúl Fernández-Lopez, Irene Del Campo, Carlos Revilla, Ana Cuevas, and Fernando de la Cruz. Negative feedback and transcriptional overshooting in a regulatory network for horizontal gene transfer. PLoS genetics, 10(2):e1004171, February 2014.

[FWG94] W Claiborne Fuqua, Stephen C Winans, and E Peter Greenberg. Quorum sensing in bacteria: the luxr-luxi family of cell density-responsive transcriptional regulators. Journal of bacteriology, 176(2):269, 1994. 
[FWS ${ }^{+}$05] Eileen Fung, Wilson W Wong, Jason K Suen, Thomas Bulter, Sun-gu Lee, and James C Liao. A synthetic gene-metabolic oscillator. Nature, 435(7038):118-122, 2005.

[GAJ13] Didier Gonze and Wassim Abou-Jaoudé. The Goodwin model: behind the Hill function. PloS One, 8(8):e69573, 2013.

[GBdlC08] M Pilar Garcillán-Barcia and Fernando de la Cruz. Why is entry exclusion an essential feature of conjugative plasmids? Plasmid, 60(1):1-18, 2008.

[GBFdlC09] María Pilar Garcillán-Barcia, María Victoria Francia, and Fernando de la Cruz. The diversity of conjugative relaxases and its application in plasmid classification. FEMS Microbiology Reviews, 33(3):657-687, may 2009.

[GCC00] T S Gardner, C R Cantor, and J J Collins. Construction of a genetic toggle switch in Escherichia coli. Nature, 403(6767):339 342,2000 .

[GdlCR13] Julien Guglielmini, Fernando de la Cruz, and Eduardo P C Rocha. Evolution of conjugation and type IV secretion systems. Molecular Biology and Evolution, 30(2):315-31, February 2013.

[GGGPdP ${ }^{+}$17] Martín Gutiérrez, Paula Gregorio-Godoy, Guillermo Pérez del Pulgar, Luis Muñoz, Sandra Sáez, and Alfonso RodríguezPatón. A New Improved and Extended Version of the Multicell Bacterial Simulator gro. ACS Synth. Biol., 2017. doi: 10.1021/acssynbio.7b00003.

[Gil77] Daniel T. Gillespie. Exact stochastic simulation of coupled chemical reactions. J. Phys. Chem., 81(25):2340-2361, Dec 1977 .

[GIPRP17] Vishal Gupta, Jesús Irimia, Iván Pau, and Alfonso RodríguezPatón. Bioblocks: Programming protocols in biology made easier. ACS Synthetic Biology, 2017. doi: 10.1021/acssynbio. 6b00304.

[GL15] Georgiev-Lab. Cece. https://github.com/GeorgievLab/ CeCe/, 2015. Accessed June 1, 2017.

[GMA15] Angel Goni-Moreno and Martyn Amos. Discus: A simulation platform for conjugation computing. In International Conference on Unconventional Computation and Natural Computation, pages 181-191. Springer, 2015.

[GMAdlC13] Angel Goñi-Moreno, Martyn Amos, and Fernando de la Cruz. Multicellular computing using conjugation for wiring. PLoS One, 8(6):e65986, 2013.

$\left[\mathrm{GMT}^{+} 12\right]$ Thomas E Gorochowski, Antoni Matyjaszkiewicz, Thomas Todd, Neeraj Oak, Kira Kowalska, Stephen Reid, Krasimira T Tsaneva-Atanasova, Nigel J Savery, Claire S Grierson, and 
Mario di Bernardo. Bsim: an agent-based tool for modeling bacterial populations in systems and synthetic biology. PLoS One, 7(8):e42790, 2012.

[Goo] Google. Blockly. https://developers.google.com/ blockly/. Accessed June 1, 2017.

[Goo65] Brian C Goodwin. Oscillatory behavior in enzymatic control processes. Advances in enzyme regulation, 3:425IN1429IN3431428IN2430IN6437, 1965.

[Gor16] Thomas E Gorochowski. Agent-based modelling in synthetic biology. Essays Biochem., 60(4):325-336, 2016.

[GRP15a] Antonio Prestes García and Alfonso Rodríguez-Patón. Bactosim-an individual-based simulation environment for bacterial conjugation. In International Conference on Practical Applications of Agents and Multi-Agent Systems, pages 275-279. Springer, 2015.

[GRP15b] Antonio Prestes García and Alfonso Rodríguez-Patón. A preliminary assessment of three strategies for the agent-based modeling of bacterial conjugation. In 9th International Conference on practical applications of computational biology and bioinformatics, pages 1-9. Springer, 2015.

[HCC $\left.{ }^{+} 16\right]$ Ferdi L Hellweger, Robert J Clegg, James R Clark, Caroline M Plugge, and Jan-Ulrich Kreft. Advancing microbial sciences by individual-based modelling. Nat. Rev. Microbiol., 14:461-471, 2016 .

$\left[\mathrm{HFS}^{+} 03\right]$ Michael Hucka, Andrew Finney, Herbert M Sauro, Hamid Bolouri, John C Doyle, Hiroaki Kitano, Adam P Arkin, Benjamin J Bornstein, Dennis Bray, Athel Cornish-Bowden, et al. The systems biology markup language (sbml): a medium for representation and exchange of biochemical network models. Bioinformatics, 19(4):524-531, 2003.

[HMC02] Jeff Hasty, David McMillen, and James J Collins. Engineered gene circuits. Nature, 420(6912):224-230, 2002.

[HRD $\left.{ }^{+} 14\right]$ William R Harcombe, William J Riehl, Ilija Dukovski, Brian R Granger, Alex Betts, Alex H Lang, Gracia Bonilla, Amrita Kar, Nicholas Leiby, and Pankaj Mehta. Metabolic resource allocation in individual microbes determines ecosystem interactions and spatial dynamics. Cell Rep., 7(4):1104-1115, 2014.

[Int] Intel. Openmp. https://www.openmprtl.org. Accessed June $1,2017$.

[JOEK12] Seunghee S Jang, Kevin T Oishi, Robert G Egbert, and Eric Klavins. Specification and simulation of synthetic multicelled behaviors. ACS Synth. Biol., 1(8):365-374, 2012. 
$\left[\mathrm{K}^{+} 01\right]$ Hiroaki Kitano et al. Foundations of systems biology. MIT press Cambridge, 2001.

[KBW98] Jan-Ulrich Kreft, Ginger Booth, and Julian WT Wimpenny. Bacsim, a simulator for individual-based modelling of bacterial colony growth. Microbiology, 144(12):3275-3287, 1998.

[KC10] Ahmad S Khalil and James J Collins. Synthetic biology: applications come of age. Nature Reviews Genetics, 11(5):367-379, 2010 .

[KK17] Takahiro Kohsokabe and Kunihiko Kaneko. Boundary-induced pattern formation from temporal oscillation: Spatial map analysis. EPL (Europhysics Letters), 116(4):48005, 2017.

$\left[\mathrm{KKA}^{+} 04\right]$ Hideki Kobayashi, Mads Kærn, Michihiro Araki, Kristy Chung, Timothy S Gardner, Charles R Cantor, and James J Collins. Programmable cells: interfacing natural and engineered gene networks. Proceedings of the National Academy of Sciences of the United States of America, 101(22):8414-8419, 2004.

[KKM $\left.{ }^{+} 14\right]$ Seunghwa Kang, Simon Kahan, Jason McDermott, Nicholas Flann, and Ilya Shmulevich. Biocellion: accelerating computer simulation of multicellular biological system models. Bioinformatics, 30(21):3101-3108, 2014.

[Klaa] Eric Klavins. Gro github. https://github.com/klavinslab/ gro. Accessed June 1, 2017.

[Klab] Eric Klavins. Gro simulation after five days. https://www . youtube.com/watch?v=P0ykJZhc0wI. Accessed June 1, 2017.

[Kla04] Eric Klavins. A language for modeling and programming cooperative control systems. In Proceedings of the IEEE International Conference on Robotics and Automation, volume 4, pages 3403-3410. IEEE, 2004.

$\left[\mathrm{KLF}^{+} 07\right]$ Stephen M Krone, Ruinan Lu, Randal Fox, Haruo Suzuki, and Eva M Top. Modelling the spatial dynamics of plasmid transfer and persistence. Microbiology, 153(8):2803-2816, 2007.

[KM10] Shigeru Kondo and Takashi Miura. Reaction-diffusion model as a framework for understanding biological pattern formation. science, 329(5999):1616-1620, 2010.

[KNSS02] Marta Kwiatkowska, Gethin Norman, Roberto Segala, and Jeremy Sproston. Automatic verification of real-time systems with discrete probability distributions. Theor. Comput. Sci., 282(1):101-150, 2002.

$\left[\mathrm{KRH}^{+} 10\right]$ Mariam Kiran, Paul Richmond, Mike Holcombe, Lee Shawn Chin, David Worth, and Chris Greenough. Flame: simulating large populations of agents on parallel hardware architectures. In Proceedings of the 9th International Conference 
on Autonomous Agents and Multiagent Systems: Volume 1, volume 1, pages 1633-1636. International Foundation for Autonomous Agents and Multiagent Systems, 2010.

[KS08] Guy Karlebach and Ron Shamir. Modelling and analysis of gene regulatory networks. Nat. Rev. Mol. Cell Biol., 9(10):770-780, oct 2008 .

[LBZG15] Rongming Liu, Marcelo C Bassalo, Ramsey I Zeitoun, and Ryan T Gill. Genome scale engineering techniques for metabolic engineering. Metabolic engineering, 32:143-154, 2015.

$\left[\mathrm{LCRP}^{+}\right.$05] Sean Luke, Claudio Cioffi-Revilla, Liviu Panait, Keith Sullivan, and Gabriel Balan. Mason: A multiagent simulation environment. Simulation, 81(7):517-527, 2005.

[LGRCC02] Matxalen Llosa, F Xavier Gomis-Rüth, Miquel Coll, and Fernando de la Cruz. Bacterial conjugation: a two-step mechanism for DNA transport. Molecular Microbiology, 45(1):1-8, 2002.

$\left[\mathrm{LMM}^{+} 11\right]$ Laurent A Lardon, Brian V Merkey, Sónia Martins, Andreas Dötsch, Cristian Picioreanu, Jan-Ulrich Kreft, and Barth F Smets. idynomics: next-generation individual-based modelling of biofilms. Environ. Microbiol., 13(9):2416-2434, 2011.

[Lod08] Harvey Lodish. Molecular Cell Biology. Macmillan, 2008.

[Lon] Imperial College London. Ecolibrium project. http://2016. igem.org/Team:Imperial_College/GRO. Accessed June 1, 2017.

[LR09] O Satya Lakshmi and NM Rao. Evolving lac repressor for enhanced inducibility. Protein Eng., Des. Sel., 22(2):53-58, 2009.

[LS] Scott Lembcke and Howling Moon Software. Chipmunk 2d physics. https://chipmunk-physics.net/. Accessed June 1, 2017.

[LW81] M W Lewis and J W Wimpenny. The influence of nutrition and temperature on the growth of colonies of Escherichia coli K12. Can. J. Microbiol., 27(7):679-684, 1981.

[MA03] Shmoolik Mangan and Uri Alon. Structure and function of the feed-forward loop network motif. Proceedings of the National Academy of Sciences, 100(21):11980-11985, 2003.

[Mar14] Luis Enrique Muñoz Martín. Nuevo algoritmo de relajación para motores de cuerpos sólidos. B.S. Thesis, Universidad Politécnica de Madrid, Madrid, España, June 2014.

[MB01] Melissa B Miller and Bonnie L Bassler. Quorum sensing in bacteria. Annu. Rev. Microbiol., 55(1):165-199, 2001. 
[MBRP11] Jesús M Miró-Bueno and Alfonso Rodríguez-Patón. A simple negative interaction in the positive transcriptional feedback of a single gene is sufficient to produce reliable oscillations. PloS One, 6(11):e27414, 2011.

[MC95] Brian Mirtich and John Canny. Impulse-based simulation of rigid bodies. In Proceedings of the Symposium on Interactive $3 D$ graphics, pages 181-189, New York, New York, USA, apr 1995. ACM Press.

[Mil07] Ian Millington. Game physics engine development. Morgan Kaufmann Publishers Amsterdam, 2007.

$\left[\mathrm{MMC}^{+} 16\right]$ Javier Macia, Romilde Manzoni, Núria Conde, Arturo Urrios, Eulàlia de Nadal, Ricard Solé, and Francesc Posas. Implementation of complex biological logic circuits using spatially distributed multicellular consortia. PLoS Comput Biol, 12(2):e1004685, 2016.

[Mon49] Jacques Monod. The growth of bacterial cultures. Annu. Rev. Microbiol., 3(1):371-394, 1949.

[MPS12] Javier Macía, Francesc Posas, and Ricard V Solé. Distributed computation: the new wave of synthetic biology devices. Trends Biotechnol., 30(6):342-349, jun 2012.

$\left[\mathrm{MPW}^{+}\right.$03] Vincent JJ Martin, Douglas J Pitera, Sydnor T Withers, Jack D Newman, and Jay D Keasling. Engineering a mevalonate pathway in escherichia coli for production of terpenoids. Nature biotechnology, 21(7):796, 2003.

$\left[\mathrm{MSOI}^{+} 02\right]$ Ron Milo, Shai Shen-Orr, Shalev Itzkovitz, Nadav Kashtan, Dmitri Chklovskii, and Uri Alon. Network motifs: simple building blocks of complex networks. Science, 298(5594):824-827, 2002 .

[MW05] Ryan McDaniel and Ron Weiss. Advances in synthetic biology: on the path from prototypes to applications. Current Opinion in Biotechnology, 16(4):476 - 483, 2005.

[NDS $\left.{ }^{+} 16\right]$ Alec AK Nielsen, Bryan S Der, Jonghyeon Shin, Prashant Vaidyanathan, Vanya Paralanov, Elizabeth A Strychalski, David Ross, Douglas Densmore, and Christopher A Voigt. Genetic circuit design automation. Science, 352(6281):aac7341, 2016 .

[NH79] KH Nealson and John W Hastings. Bacterial bioluminescence: its control and ecological significance. Microbiological reviews, 43(4):496, 1979.

$\left[\mathrm{NMDV}^{+}{ }^{17}\right]$ Isaac N. Nuñez, Tamara F. Matute, Ilenne D. Del Valle, Anton Kan, Atri Choksi, Drew Endy, Jim Haseloff, Timothy J. Rudge, and Fernan Federici. Artificial symmetry-breaking for morphogenetic engineering bacterial colonies. ACS Synthetic Biology, 6(2):256-265, 2017. PMID: 27794593. 
[NPH70] Kenneth H Nealson, Terry Platt, and J Woodland Hastings. Cellular control of the synthesis and activity of the bacterial luminescent system. Journal of bacteriology, 104(1):313-322, 1970.

[OE12] Monica E Ortiz and Drew Endy. Engineered cell-cell communication via DNA messaging. J. Biol. Eng., 6(1):16, jan 2012.

$\left[\mathrm{OFPF}^{+} 17\right]$ James M Osborne, Alexander G Fletcher, Joe M Pitt-Francis, Philip K Maini, and David J Gavaghan. Comparing individualbased approaches to modelling the self-organization of multicellular tissues. PLoS computational biology, 13(2):e1005387, 2017.

[OK14] Kevin Oishi and Eric Klavins. Framework for engineering finite state machines in gene regulatory networks. ACS synthetic biology, 3(9):652-665, 2014.

[PB16] Kai Papenfort and Bonnie L Bassler. Quorum sensing signalresponse systems in gram-negative bacteria. Nature Reviews Microbiology, 14(9):576-588, 2016.

[PE85] Dirk-Jan Povel and Peter Essens. Perception of temporal patterns. Music Perception: An Interdisciplinary Journal, 2(4):411-440, 1985.

[PGRP16] Antonio Prestes García and Alfonso Rodríguez-Patón. Sensitivity analysis of repast computational ecology models with r/repast. Ecology and Evolution, 6(24):8811-8831, 2016.

$\left[\mathrm{PPK}^{+} 16\right]$ Jonathan Pascalie, Martin Potier, Taras Kowaliw, Jean-Louis Giavitto, Olivier Michel, Antoine Spicher, and René Doursat. Developmental design of synthetic bacterial architectures by morphogenetic engineering. ACS Synth. Biol., 5(8):842-861, 2016.

[PW09] Priscilla EM Purnick and Ron Weiss. The second wave of synthetic biology: from modules to systems. Nature Reviews Molecular Cell biology, 10(6):410-422, 2009.

$\left[\mathrm{QCIA}^{+}\right.$15] Jacqueline Y Quinn, Robert Sidney Cox III, Aaron Adler, Jacob Beal, Swapnil Bhatia, Yizhi Cai, Joanna Chen, Kevin Clancy, Michal Galdzicki, Nathan J Hillson, et al. Sbol visual: a graphical language for genetic designs. PLoS Biol, 13(12):e1002310, 2015 .

$\left[\mathrm{R}^{+} 70\right]$ Winston W Royce et al. Managing the development of large software systems. In proceedings of IEEE WESCON, volume 26, pages 1-9. Los Angeles, 1970.

$\left[\mathrm{RBC}^{+} 16\right]$ Nicholas Roehner, Jacob Beal, Kevin Clancy, Bryan Bartley, Goksel Misirli, Raik Grünberg, Ernst Oberortner, Matthew Pocock, Michael Bissell, Curtis Madsen, Tramy Nguyen, Michael Zhang, Zhen Zhang, Zach Zundel, Douglas Densmore, 
John H. Gennari, Anil Wipat, Herbert M. Sauro, and Chris J. Myers. Sharing structure and function in biological design with SBOL 2.0. ACS Synth. Biol., 5(6):498-506, 2016.

[RFS $\left.{ }^{+} 13\right]$ Timothy J Rudge, Fernán Federici, Paul J Steiner, Anton Kan, and Jim Haseloff. Cell polarity-driven instability generates selforganized, fractal patterning of cell layers. ACS synthetic biology, 2(12):705-714, 2013.

[RG10] Junuthula Narasimha Reddy and David K Gartling. The Finite Element Method in Heat Transfer and Fluid Dynamics. CRC press, 2010.

$\left[\mathrm{RMC}^{+} 11\right]$ Sergi Regot, Javier Macia, Núria Conde, Kentaro Furukawa, Jimmy Kjellén, Tom Peeters, Stefan Hohmann, Eulàlia de Nadal, Francesc Posas, and Ricard Solé. Distributed biological computation with multicellular engineered networks. Nature, 469(7329):207-211, 2011.

$\left[\mathrm{RPO}^{+} 06\right]$ Dae-Kyun Ro, Eric M Paradise, Mario Ouellet, Karl J Fisher, Karyn L Newman, John M Ndungu, Kimberly A Ho, Rachel A Eachus, Timothy S Ham, James Kirby, et al. Production of the antimalarial drug precursor artemisinic acid in engineered yeast. Nature, 440(7086):940-943, 2006.

[RSLY12] Katherine A Riccione, Robert P Smith, Anna J Lee, and Lingchong You. A synthetic biology approach to understanding cellular information processing. ACS synthetic biology, 1(9):389402, 2012.

[RSPH12] Timothy J Rudge, Paul J Steiner, Andrew Phillips, and Jim Haseloff. Computational modeling of synthetic microbial biofilms. ACS Synth. Biol., 1(8):345-352, 2012.

[RVMR99] Peter Ruoff, Merete Vinsjevik, Christian Monnerjahn, and Ludger Rensing. The Goodwin oscillator: on the importance of degradation reactions in the circadian clock. Journal of biological rhythms, 14(6):469-479, 1999.

[SADN $\left.{ }^{+} 16\right]$ Ricard Solé, Daniel R Amor, Salva Duran-Nebreda, Núria Conde-Pueyo, Max Carbonell-Ballestero, and Raúl Montañez. Synthetic collective intelligence. Biosystems, 148:47-61, 2016.

[Sáe15] Sandra Sáez. Diseño e implementación de un módulo de crecimiento bacteriano dependiente de nutrientes en el simulador gro. B.S. Thesis, Universidad Politécnica de Madrid, Madrid, España, July 2015.

[SBB00] Paul Smolen, Douglas A Baxter, and John H Byrne. Mathematical modeling of gene networks. Neuron, 26(3):567-580, 2000 . 
$\left[\mathrm{SBH}^{+} 05\right]$ Søren J Sørensen, Mark Bailey, Lars H Hansen, Niels Kroer, and Stefan Wuertz. Studying plasmid horizontal transfer in situ: a critical review. Nature Reviews Microbiology, 3(9):700710, 2005

$\left[\mathrm{SCB}^{+} 08\right]$ Jesse Stricker, Scott Cookson, Matthew R Bennett, William H Mather, Lev S Tsimring, and Jeff Hasty. A fast, robust and tunable synthetic gene oscillator. Nature, 456(7221):516-519, 2008.

$\left[\mathrm{SGBF}^{+} 10\right]$ Chris Smillie, M Pilar Garcillán-Barcia, M Victoria Francia, Eduardo P C Rocha, and Fernando de la Cruz. Mobility of plasmids. Microbiol. Mol. Biol. Rev., 74(3):434-452, September 2010 .

[SH11] Matthew Scott and Terence Hwa. Bacterial growth laws and their applications. Current Opinion in Biotechnology, 22(4):559-565, 2011.

[SH16] Spencer R Scott and Jeff Hasty. Quorum sensing communication modules for microbial consortia. ACS Synth. Biol., 5(9):969977, 2016.

[SHP ${ }^{+}$09] Takayuki Sohka, Richard A Heins, Ryan M Phelan, Jennifer M Greisler, Craig A Townsend, and Marc Ostermeier. An externally tunable bacterial band-pass filter. Proceedings of the $\mathrm{Na}$ tional Academy of Sciences, 106(25):10135-10140, 2009.

[SLR ${ }^{+}$12] Pin-Tzu Su, Chih-Tang Liao, Jiunn-Ren Roan, Shao-Hung Wang, Arthur Chiou, and Wan-Jr Syu. Bacterial colony from two-dimensional division to three-dimensional development. PloS One, 7(11):e48098, 2012.

$\left[\mathrm{SMG}^{+} 14\right]$ Yolanda Schaerli, Andreea Munteanu, Magüi Gili, James Cotterell, James Sharpe, and Mark Isalan. A unified design space of synthetic stripe-forming networks. Nature Communications, $5,2014$.

[SOMMA02] Shai S Shen-Orr, Ron Milo, Shmoolik Mangan, and Uri Alon. Network motifs in the transcriptional regulation network of escherichia coli. Nature genetics, 31(1):64-68, 2002.

[Sto02] Mariëlle Stoelinga. An introduction to probabilistic automata. Bull. Eur. Assoc. Theor. Comput. Sci. EATCS, 78(176-198):2, 2002 .

$\left[\mathrm{TKM}^{+}\right.$09] R. Tokita, T Katoh, Y. Maeda, J. Wakita, M. Sano, T. Matsuyama, and M. Matsushita. Pattern Formation of Bacterial Colonies by Escherichia coli. J. Phys. Soc. Jpn., 78(7):074005, 2009 .

[TL47] EL Tatum and Joshua Lederberg. Gene recombination in the bacterium escherichia coli. Journal of bacteriology, 53(6):673, 1947. 
[TMLSF09] Marcel Tigges, Tatiana T Marquez-Lago, Jörg Stelling, and Martin Fussenegger. A tunable synthetic mammalian oscillator. Nature, 457(7227):309-312, 2009.

[TSS $\left.{ }^{+} 09\right]$ Jeffrey J Tabor, Howard M Salis, Zachary Booth Simpson, Aaron a Chevalier, Anselm Levskaya, Edward M Marcotte, Christopher A Voigt, and Andrew D Ellington. A synthetic genetic edge detection program. Cell, 137(7):1272-1281, jun 2009 .

[TTV11] Alvin Tamsir, Jeffrey J Tabor, and Christopher A Voigt. Robust multicellular computing using genetically encoded nor gates and chemical 'wires'. Nature, 469(7329):212-215, 2011.

[Tur37] Alan M Turing. Computability and $\lambda$-definability. The Journal of Symbolic Logic, 2(4):153-163, 1937.

[TW04] Seth Tisue and Uri Wilensky. Netlogo: A simple environment for modeling complexity. In International Conference on Complex Systems, volume 21, pages 16-21. Boston, MA, 2004.

[VCHT08] Dmitri Volfson, Scott Cookson, Jeff Hasty, and Lev S Tsimring. Biomechanical ordering of dense cell populations. Proc. Natl. Acad. Sci. U. S. A., 105(40):15346-15351, oct 2008.

[WB02] Ron Weiss and Subhyu Basu. The device physics of cellular logic gates. In NSC-1: The First Workshop of Non-Silicon Computing. Boston, Massachusetts, 2002.

[WB05] Christopher M Waters and Bonnie L Bassler. Quorum sensing: cell-to-cell communication in bacteria. Annu. Rev. Cell Dev. Biol., 21:319-346, 2005.

$\left[\mathrm{WBH}^{+}\right.$03] Ron Weiss, Subhayu Basu, Sara Hooshangi, Abigail Kalmbach, David Karig, Rishabh Mehreja, and Ilka Netravali. Genetic circuit building blocks for cellular computation, communications, and signal processing. Natural computing, 2(1):47-84, 2003.

$\left[\mathrm{WBS}^{+}\right.$01] Neil A Whitehead, Anne ML Barnard, Holly Slater, Natalie JL Simpson, and George PC Salmond. Quorum-sensing in gramnegative bacteria. FEMS microbiology reviews, 25(4):365-404, 2001.

[WRI ${ }^{+}$98] Jun-ichi Wakita, Ismael Rafols, Hiroto Itoh, Tohey Matsuyama, and Mitsugu Matsushita. Experimental Investigation on the Formation of Dense-Branching-Morphology-Like Colonies in Bacteria. Journal of the Physical Society of Japan, 67(10):36303636, 1998.

[YCWA04] Lingchong You, Robert Sidney Cox, Ron Weiss, and Frances H Arnold. Programmed population control by cell--cell communication and regulated killing. Nature, 428(6985):868-871, 2004. 
[YNFR $\left.{ }^{+} 14\right]$ Lei Yang, Alec AK Nielsen, Jesús Fernández-Rodriguez, Conor J McClune, Michael T Laub, Timothy K Lu, and Christopher A Voigt. Permanent genetic memory with > 1-byte capacity. Nature methods, 11(12):1261-1266, 2014.

$\left[\mathrm{ZDF}^{+} 12\right]$ Xue Zhong, Jason Droesch, Randal Fox, Eva M Top, and Stephen M Krone. On the meaning and estimation of plasmid transfer rates for surface-associated and well-mixed bacterial populations. J. Theor. Biol., 294:144-152, February 2012.

[ZJRVR90] MH Zwietering, Il Jongenburger, FM Rombouts, and K Van't Riet. Modeling of the bacterial growth curve. Applied and environmental microbiology, 56(6):1875-1881, 1990. 


\section{Appendix A}

\section{ProSpec specification reference}

The way of specifying simulations has been adapted to respond to a new paradigm in which structure and interactions of genetic circuits are stated (and behavior emerges). Other features and parameters that drive the simulations such as actions, variables and functions may also be part of this new specification. The new specification of GRO simulations is also compatible with the guarded command paradigm. In this document, the new syntax for simulation specifications will be described. Also, the new features will be identified to fully define the capabilities of the system.

\section{A.1 A GRO specification (with its new syntax)}

A GRO specification is usually written in four parts:

1. Library inclusion, parameter setting and definition of global variables and values

2. Genetic entities and their relationships

3. Actions associated to protein conditions

4. Top level programs (main function and other functions) and global control

Let's take a look into how these parts should be written.

\section{A.1.1 Library inclusion, parameter setting, definition of global variables and values}

Generally, library inclusion is standard and is covered by:

include gro 
That instruction is the first one that should be written in any specification. All of the instructions of the specification language may be used once this instruction has been added to the specification. Other libraries may be included by using the keyword include, however not many libraries have been developed for our new specification yet.

Once the libraries have been included in the specification, the parameters for the simulation must be set. To set the parameter values, the keyword set is used as in the following example, in which the duration of the timestep is set to 0.1 minutes:

set("dt", 0.1);

In the context of parameters, the specification syntax has two new groups of parameters. The first group is the group of parameters that control the nutrients in the simulator. The other group are the parameters and settings relative to environmental signal control in the simulation.

It is important to note that not all parameters have to be set explicitly. All of the parameters have a default value. Parameters must only be set when a specific value is needed for the parameter and that parameter will be used. In all of the examples in this document, an arbitrary value is set, just for the sake of exemplification.

The parameters for the nutrient module are the following ones. The description for each parameter is in a comment at the end of each line:

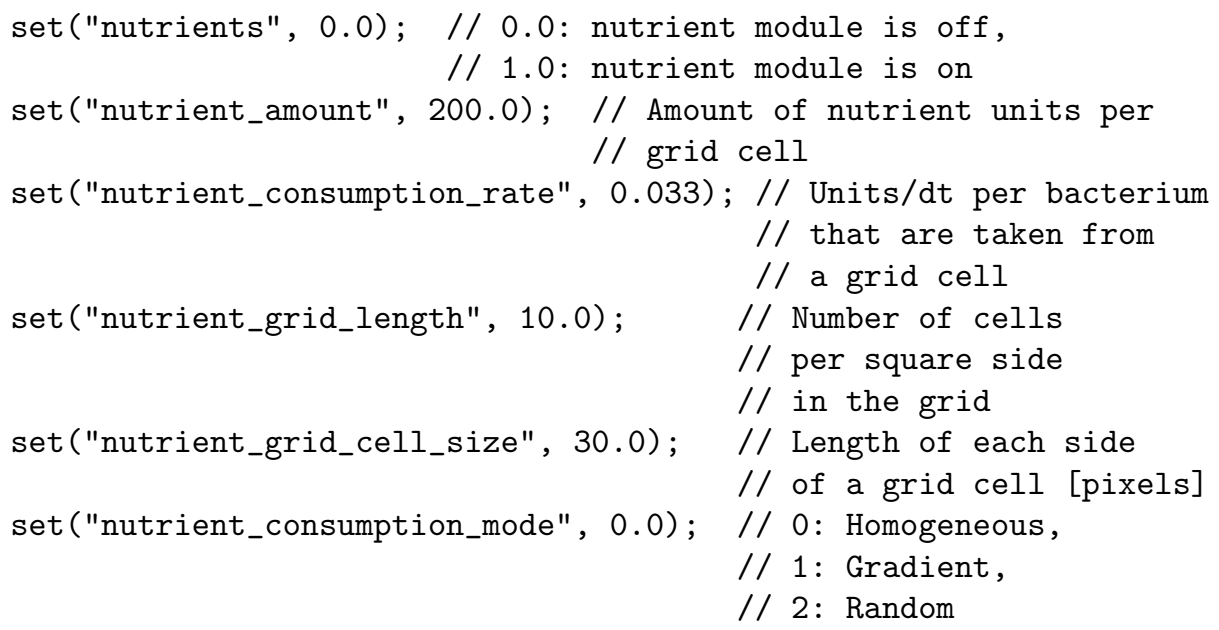

In the case of environmental signal control, the following parameters can be set:

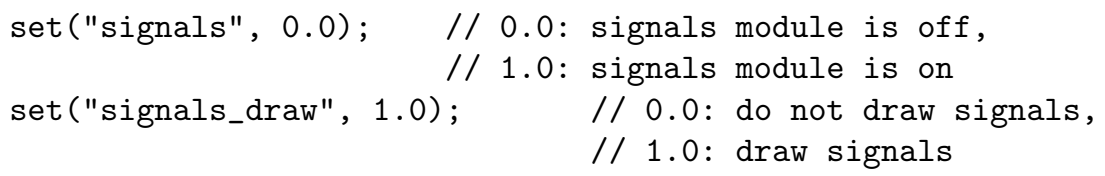


Any signal definition should be placed after setting all the parameter values. In the following example, a signal identified by the symbol $\mathrm{s} 1$ is defined and its parameters (diffusion coefficient, degradation coefficient and maximum concentration, respectively) are set:

// Grid settings (type of grid, diffusion method,

// grid length, grid cell size and neighborhood size)

// and signal parameters: diffusion, degradation.

grid("continuous", "gro_original", 10, 10, 8);

s1 := s_signal ([kdiff $:=0.02, \operatorname{kdeg}:=0.005])$;

Further along the specification, the symbol s1 will always be used to refer to this signal. Examples of operations on these signals will be shown when we reach the action section of the specification.

Any other global variable may be defined at this stage also (such as a global time counter, strings that represent a route or filename, flags, for example), although it is not mandatory to do it in this section.

Once this section is defined, the next part of the specification consists of the genetic elements that will participate in the simulation.

\section{A.1.2 Genetic entities and their relationships}

The next section defines the core of the simulation. It specifies all of the genetic elements participating in the simulation, their dynamics and the relationships between them. Since the goal is to achieve a configurable environment, the vocabulary for names of genetic entities is open. This means that the programmer may choose a name for the elements, and that name shall act as an identifier throughout the specification. Therefore, the first step in this section is to identify the genetic elements, and define their dynamics and interactions.

An operon is a set of genes under the control of a single promoter. In this context, the operon is an intermediate stage of grouping, whereas the highest level of grouping comes in the form of plasmids. Both types of grouping are necessary to the specification and will be shown in this document.

Operon definition is done through the keyword genes. Each operon definition may hold a lot of information related to the operon, however, it is important to note that it is not mandatory to fill in all of the information. Whatever information is important should be filled in, and what is not specified takes a default value. Mandatory fields are indicated below. 
An example of operon specification is now shown and will be explained:

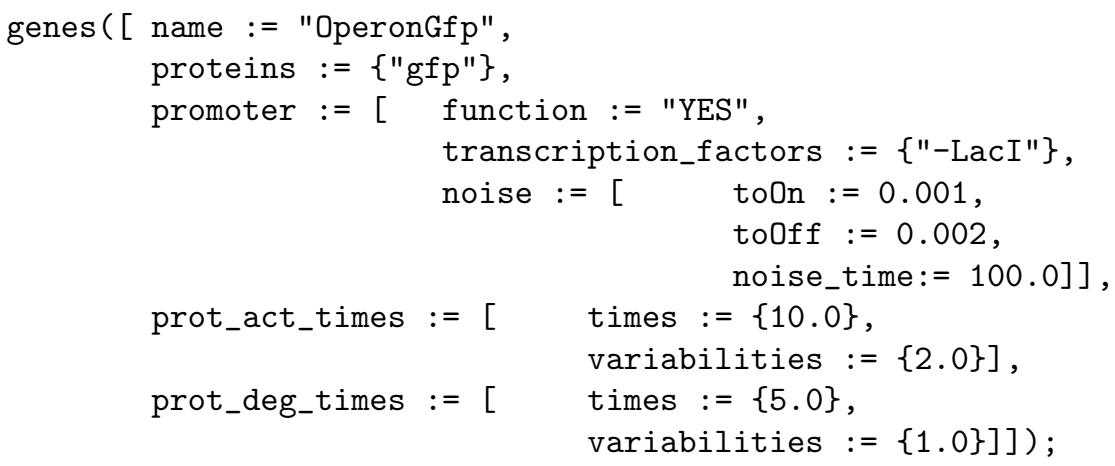

This is a complete example of how an operon is defined. The information held by an operon is the following:

- name: A name is assigned to the operon as an id. This id will later be used for setting up plasmids containing these operons. MANDATORY.

- proteins: This field lists the output proteins that the operon expresses. Usually, the operon expresses at least one protein. The proteins expressed by the operon are listed by their name. MANDATORY.

- promoter: This is a "superfield", in the form of a record, that includes several configurable features of the promoter controlling the operon. The superfield itself is MANDATORY. The subfields composing promoter are described here:

- function: Sets the logic function according to which the promoter will operate. This function may be set to YES, NOT, TRUE, FALSE, OR, XOR, AND or NAND. Any logic function may take any number of transcription_factor as input to the promoter. If this subfield is skipped, it is set to the default value YES.

- transcription_factors: It is a list of proteins that acts as the inputs for the promoter. These proteins are referred to by their name. The list may be of any size. Generally, this subfield is MANDATORY, however, when a TRUE or FALSE gate are defined for the function subfield, no transcription_factors are required. Additionally, it is possible to specify the need for the absence of a protein by adding a - in front of the protein in question, like in the example ("-LacI").

- noise: This subfield is a list of probabilities of failure of normal behavior. This is: the necessary conditions for the output proteins to be expressed at the current operon may be met, but still these proteins could fail to be expressed due to noise. Two failure cases may be set. The promoter is permanently set to off or it is permanently set to on. toOff and toOn are the respective probabilities of occurrence over a time span of noise_time. By default, these three subfield values are set to 0 . Note that to0ff and toOn may go from 0.0 to 1.0 . 
- prot_act_times: This field specifies a list of protein expression times for the operon and their variabilities (in minutes). MANDATORY. In the example, activation times are specified for each of the proteins stated in the instructions. The first part (times) specifies protein activation times and summarizes to the following: "Protein gfp has an activation mean time of 10.0 minutes and a standard deviation of 2.0 minutes". It is possible to skip the variability definition altogether and have genetic elements activate always exactly at their average activation time.

- prot_deg_times: Very much like in the case of prot_act_times, this field specifies a list of protein degradation times for the proteins and the variabilities (in minutes) of the times. MANDATORY. Note: In case several different prot_deg_times are specified for the same protein, the lowest one will be the used one for all of the same proteins.

The user may define as many operons as necessary. Once these operons are defined, they should be organized in plasmids. A plasmid may include any number of operons. The association between plasmids and operons (genes) is done through an instruction called plasmids_genes. This instruction defines a list of operons associated to each plasmid. The plasmids will be identified by their names, and so will the operons.

An example is shown for clarity:

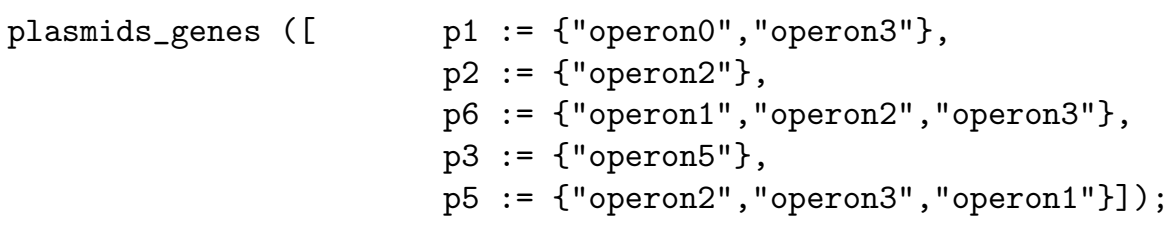

In this example, five plasmids are assigned different operons. Note that the same operon may be assigned to several plasmids.

As the last feature of genetic elements, environmental signals will be mentioned. Signals are transferred to a genetic element in the form of a transcription_factor. Hence, a signal may be a trigger for the function of an operon. How the transfer is carried out will be discussed further along this document in the next section, related to actions. 


\section{A.1.3 Actions associated to protein conditions}

Once the core of the elements and their interactions are in place, the actions that are executed by bacteria must be specified. The way in which this is done is by linking an action to a specific protein condition. The condition is expressed as a set composed of boolean states corresponding to protein expression. This represents the fact that whenever the condition is met, it will fire a certain action with its parameters. The keyword action is used to define the instruction linked to the condition. An example will now be shown and explained, and then all currently implemented actions will be listed:

action (\{"-ara", "tetR"\}, "lose_plasmid" ,\{"p5"\});

This clause states that whenever the protein ara is absent (-ara) and protein tet $R$ is present in a bacterium, plasmid p5 is removed from the list of plasmids of the bacterium. The list containing p5 is the list of parameters associated to the action lose_plasmid. This parameter list is always passed in the form of a list of strings. Whichever protein that is NOT explicitly mentioned in the condition, is assumed to be of status "don't care". This means, it does not affect the evaluation of the logical condition. Protein ids should come from the glossary and the specific actions to be executed will be listed now along with the parameters they take in:

1. paint (green, red, yellow, cyan): This action paints the bacterium according to the color combination given by the four color channels green, red, yellow and cyan. Valid values for these channels are integer numbers ranging from 0 to 50 (this is defined in gro.gro, these thresholds can be changed). Note that this instruction is absolute and imposes the color combination without regard for the previous color state in the bacterium.

2. d_paint (green, red, yellow, cyan): Unlike the previous action, this one adds (or subtracts) a certain amount of "channel concentration". This is, it modifies the previous color distribution in the bacterium by adding or subtracting a difference in each of the channels. As in the previous case, the channels may take values ranging from 0 to 50 (integer).

3. die: This action kills the bacterium and makes it disappear from the simulation space. At this time, it is the only action that does not take a parameter list in.

4. conjugate (plasmid, rate): action that copies a plasmid from a source bacterium (the one executing the action) to a random neighboring bacterium. This copy is done at an average rate, given by the average number of conjugations that occur during the lifetime of a bacterium.

5. conjugate_directed (plasmid, rate): Similar to the previous action, except that it considers a mechanism (called eex) in the destination bacterium that may not allow plasmid entry. Whenever entry is closed to the plasmid in the destination bacterium, this destination bacterium is not considered as a viable neighbor and therefore, the destination selection is directed toward all other viable neighbors. The rate parameter in this action has the same meaning as in the conjugate action. 
6. lose_plasmid (plasmid): action that removes plasmid from the list of plasmids residing in the bacterium.

7. set_eex (plasmid): Sets a constraint against plasmid entering the current bacterium (by conjugation).

8. remove_eex (plasmid): Removes the constraint placed upon plasmid that forbids its entry to the current bacterium (by conjugation).

9. set_growth_rate (rate): Sets the rate at which a bacterium will grow. This rate is given in $\mu \mathrm{m} / \mathrm{min}$.

10. emit_cf (signal_id, concentration): action that commands the current bacterium to emit concentration amount of the cross-feeding signal identified by signal_id.

11. get_cf (signal_id, benefit): This action reads the concentration of cross-feeding signal signal_id in the medium. Depending on the value of the benefit parameter (1 simbolizes a positive interaction -growth increases- with the signal, -1 simbolizes a negative interaction -growth decreases-), the growth rate of the bacterium will be changed.

12. s_emit_signal (signal_id, concentration, emission_type): This action is associated to the emission of a signal (identified by signal_id) concentration into the environment by a bacterium. It is emitted according to an emission_type, being "exact", "area" or "average". The first type emits the exact concentration only at the cell under the center of the bacterium, the second one emits the given concentration to all grid cells in the area under the bacterium and the third mode emits to this same area, but the amount is an average concentration over the number of cells. These modes always have the same range meaning for all signal actions, but of course refer to the specific action.

13. s_absorb_signal (signal_id, concentration, absorption_type): action that prompts the absorption of a given signal (identified by signal_id) concentration from the environment. Whenever absorption is executed, the given concentration amount is absorbed, but if there is not enough signal at the specified location (or locations), whatever present concentration is absorbed. In addition, a fourth type is included in this case: random. This absorption_type takes signal concentration from a randomly chosen cell in the area under the bacterium.

14. s_get_signal (signal_id): senses the concentration of a given signal (identified by signal_id) from the environment.

15. s_set_signal (signal_id, concentration, $\mathrm{x}, \mathrm{y}$ ): Emission of a determinate concentration of a given signal(identified by signal_id) into the environment (at location $(\mathrm{x}, \mathrm{y})$ ).

16. s_emit_cf (signal_id, concentration, emission_type): Refers to the emission of a signal (identified by signal_id) into the environment by 
a given concentration. This directive is related to cross-feeding experiments, meaning the emitted concentration is scaled by a detrimental/beneficial coefficient that is associated to the bacterium's metabolism. The emission_types can be: "exact", "area" or "average".

17. s_absorb_cf(signal_id, concentration, benefit, absorption_type): Absorbs a certain concentration of signal (identified by signal_id) from the environment. This kind of absorption affects cross-feeding experiments. The absorbed concentration triggers a response (represented by benefit: -1 is a detrimental effect and 1 is a beneficial effect) in the metabolism of the bacterium that absorbs. The absorbed amount of signal modulates the effect. Signal can be absorbed in four types: "exact", "area", "average" or "random".IMPORTANT NOTE: Using several signals to directly modulate growth of a cell affects the cross-feeding signal emission and in consequence growth. Independent modulation passing through a logic gate is how multiple cross-feeding signal growth modulation should be done.

18. s_get_cf(signal_id, concentration, benefit): Reads a certain concentration of signal (identified by signal_id) from the environment. This kind of absorption affects cross-feeding experiments. The read concentration triggers a response (represented by benefit: -1 is a detrimental effect and 1 is a beneficial effect) in the metabolism of the bacterium that is sensing. The read amount of signal modulates the effect. The IMPORTANT NOTE from s_absorb_cf holds for this action as well.

19. s_absorb_QS(signal_id, comparison, threshold, protein): Absorbs a certain amount of signal (identified by signal_id) from the environment. If the absorbed amount satisfies a condition given by a comparison sign ("<" or ">") and a threshold, the genetic element protein is activated. Otherwise, protein is deactivated. VERY IMPORTANT NOTE: any protein defined in an action of this kind CANNOT be used in action conditions.

20. S_get_QS (signal_id, comparison, threshold, protein): Is the same action as the above, except that it does not absorb signal from the environment and only senses. The VERY IMPORTANT NOTE holds for proteins defined by this action as well.

Several other actions may be implemented in the future, such as CRISPR related functions or phage control. It is very important to note that despite defining a new specification paradigm for controlling the bacteria in the simulations, this paradigm is still compatible with the previous one defined through guarded commands (meaning all (but one, due to constraints of the physical engine - chemostat mode) of the instructions that come from the original GRO still work within the scope of programs. 


\section{A.1.4 Top-level programs and global control}

This section will simply be a reminder, since the definition of top-level programs and global control come from the original GRO specification. Programs are code spaces where conditions are evaluated and instructions are executed in response to these conditions (this is the guarded command paradigm). Most of the time, programs are assigned to a bacterium as their behavior.

Another important program is the main program. The main program controls the environment and the top level settings of the simulator. Examples of these settings may be the data gathering, iteration control of simulations, bacteria creation and placement. The only difference in the pre-existing instructions lie in the bacterial creation. The instructions ecoli and $c_{-}$ecolis have been modified to include more information to initialize bacteria in the simulation space. An example of each instruction will be shown now and will be briefly explained:

ecoli([y := 20, $\mathrm{x}:=50],\{" \mathrm{p} 1 "\}, \operatorname{program} \mathrm{p}())$;

ecoli $([y:=20, x:=50]$, program $p())$;

This instruction creates a single ecoli agent. It receives two or three parameters. The first parameter is a record that specifies the physical definitions of the bacterium. These variables are: $\mathrm{x}, \mathrm{y}$, theta and volume (all float values). Next (optional) is the plasmid_list parameter that specifies what plasmids are initially present in the bacterium. The plasmids, as always, are identified through their name (id). Finally, a program to run is associated to the bacterium. Note that when the new paradigm is used, this program is usually empty $(\operatorname{skip}() ;)$.

c_ecolis $(200,0,0,200,\{" p 1 "\}$, program $p())$;

c_ecolis is an instruction that creates a group of bacteria in a random circular pattern in batch. Only the four first parameters will be described, as the two last ones are the same final parameters of the ecoli instruction with 3 parameters. The first parameter is $\mathrm{n}$ (integer). It determines the number of ecolis to create. The two following parameters are $\mathrm{x}$ and $\mathrm{y}$ (both floats), and establish where the circular pattern will be centered in the simulation space. The fourth parameter is the maximum radius in pixels (float) of the circular pattern up to where bacteria may be placed. The rest of the parameters have already been explained for the previous instruction and have the same meaning in this instruction. 


\section{A.2 Basic specification examples}

A couple of real examples will be appended to demonstrate how all of the syntax discussed in this document may be used.

The first example, newLtest.gro, is a specification in which the features of the language are tested. It was designed as the skeleton for building the new specification language. Specifically, there is a large list of parameter setting, data collection (stored in files), circuit definition (very extensive, using most of the capabilities of the specification) and a showcase of several options of actions.

\section{A.2.1 newLtest.gro}

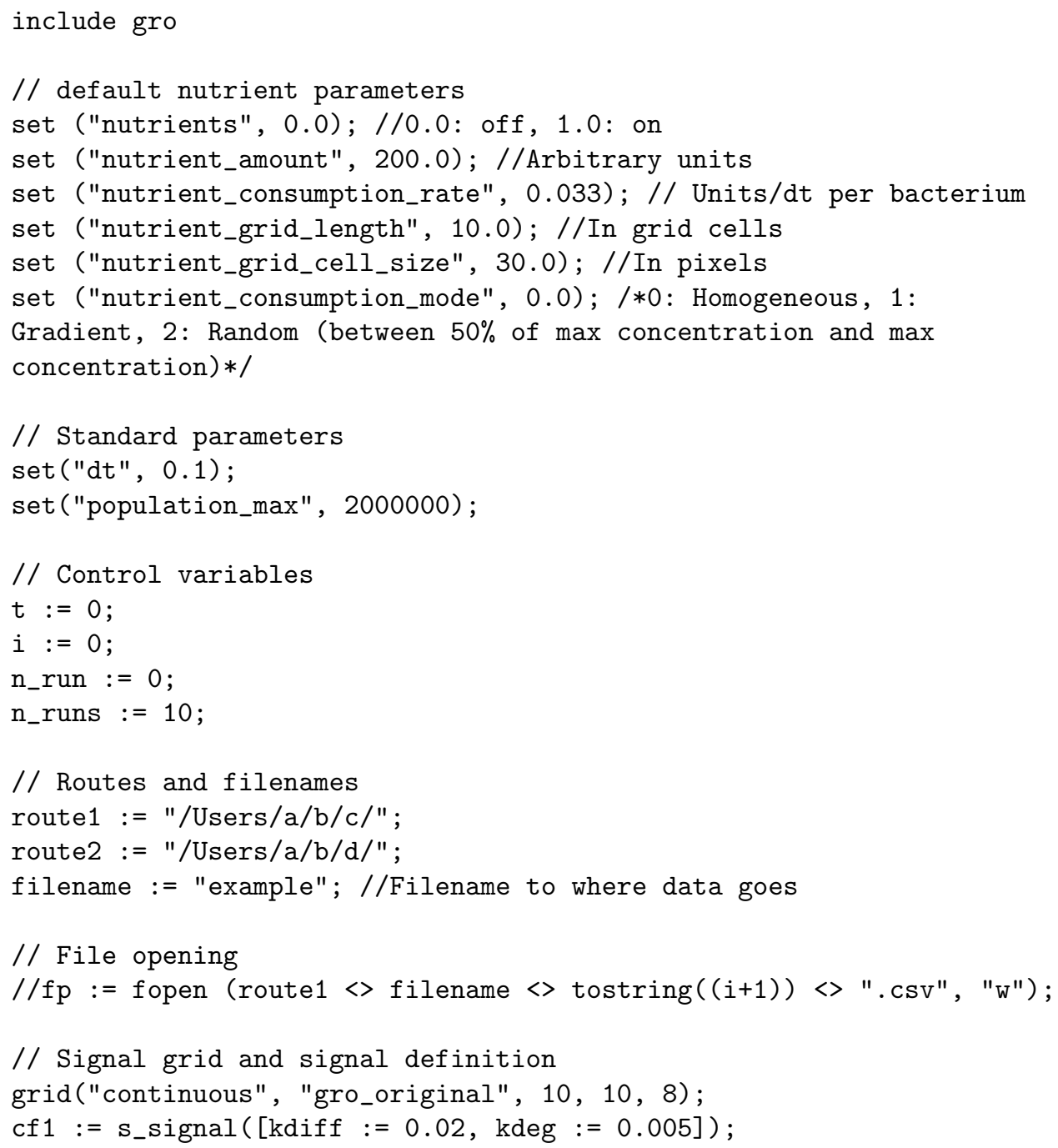


/* Example of cell program using selected cell for single dump (to file $\mathrm{fp}) * /$

/*program $\mathrm{p}():=$

\{

selected:

\{

\}

dump_single (fp) ;

\}$; * /$

//Empty program example

program $\mathrm{p}():=$

\{

\}

$\operatorname{skip}()$

// Movie program example

/*program movie() :=

\{

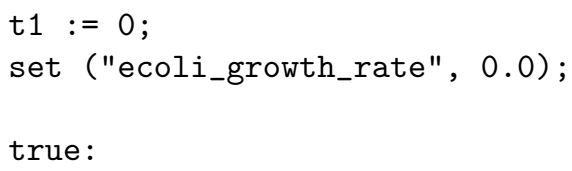


// Plasmid inclusion matrix (of operons)

plasmids_genes ( [

p1 := \{"operon0", "operon3"\},

p2 := $=\{$ "operon $2 "\}$,

p6 := "operon1", "operon2", "operon3" $\}$,

p3 $:=\{$ "operon5" $\}$,

p5 := \{"operon2", "operon3", "operon1"\}]);

// Example of how to use several actions.

action ( $\{$ "gfp" \}, "paint" , \{"500", "0", "0", "0" $\})$;

action (\{"-gfp" $\},$ "d_paint", \{"-1", "0", "0", "0" $\})$;

action ( $\{$ "ara" $\}$, "conjugate" , \{"p5", "1.0" $\})$;

action ( $\{$ lacI", "cI"\}, "die", \{"0"\});

action (\{"-ara", "tetR" $\},$ "lose_plasmid" , \{"p5"\});

action (\{"gfp"\}, "set_growth_rate", \{"0.011"\});

action (\{"-tetR", "-ara"\}, "s_emit_cf", \{tostring (cf1), "10.0" , "exact"\});

action (\{"tetR"\}, "s_get_cf", \{tostring (cf1), "5", "-1"\});

// Example of main program

/*program main() :=

\{

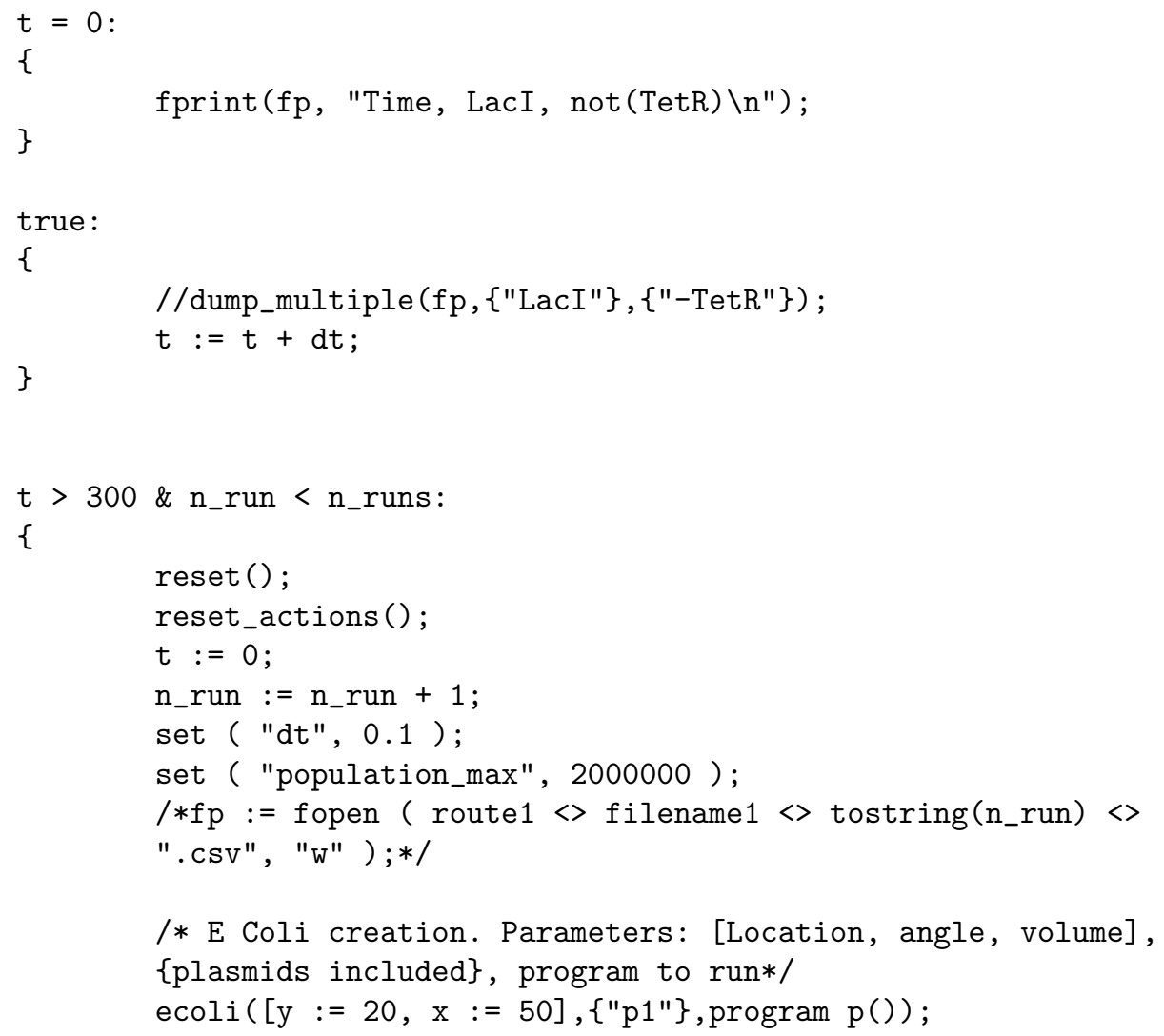




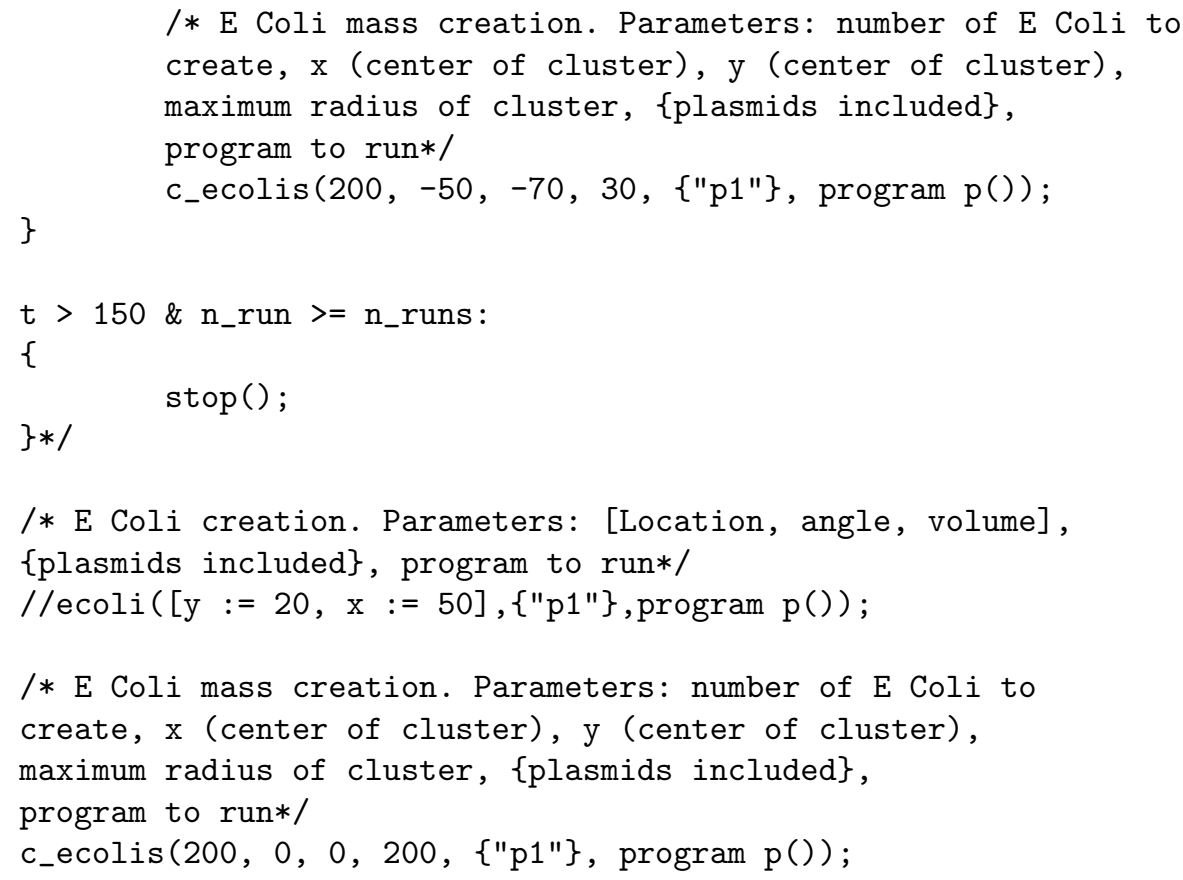


The second example is meant to test the implementation of the promoters for a circuits that have different actions associated to them, such as glowing or dying. Data is also collected in this example, and action showcase is present, although not all actions are used.

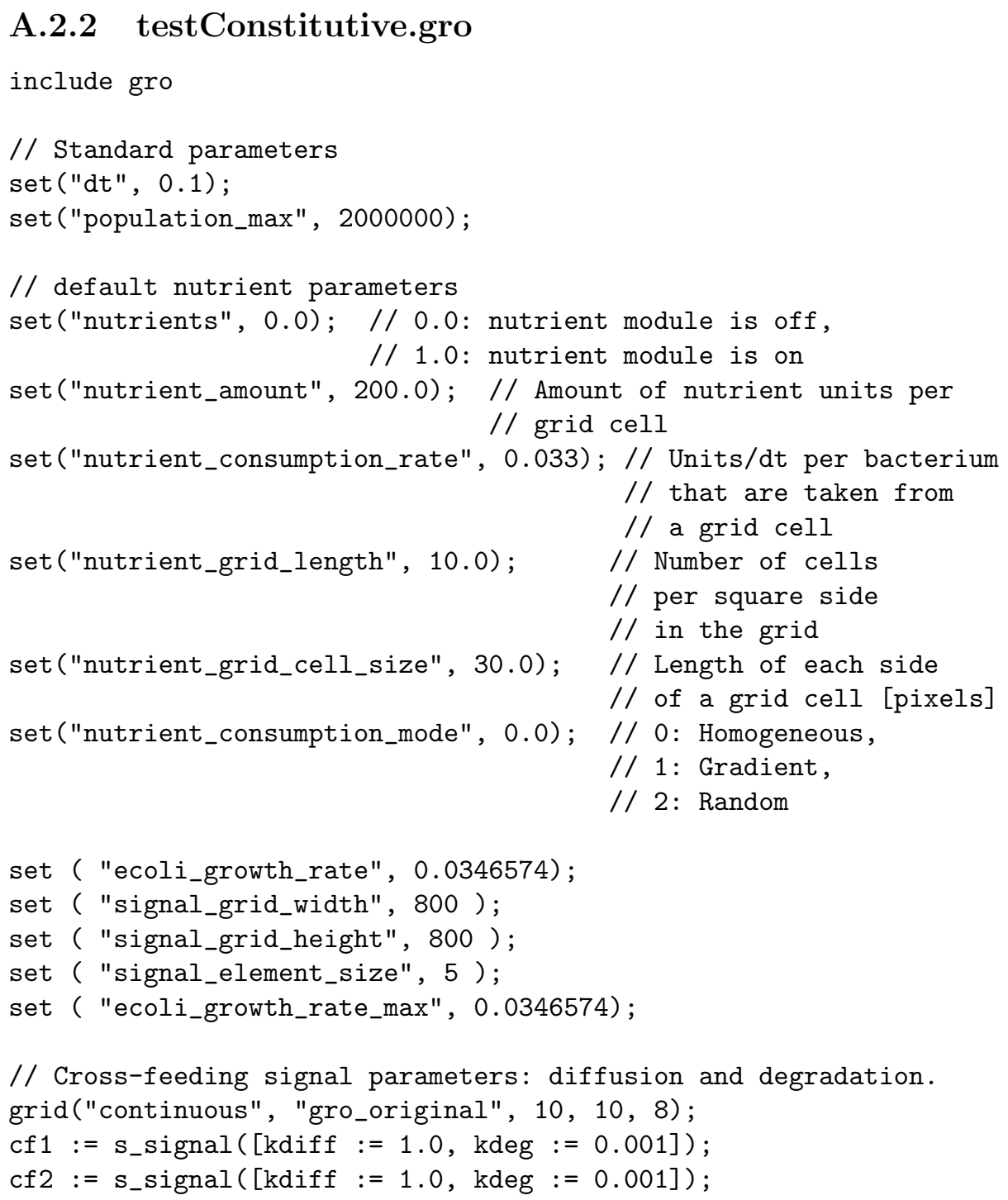


// Operon definition

genes([ name := "Operon1",

proteins := "gfp", "rel1" $\}$,

promoter $:=[$ function $:=$ "OR",

transcription_factors := "lacI", "-rel2" $\}$,

prot_act_times $:=$ [times $:=\{0.5,20.0\}$ ],

prot_deg_times $:=$ [times $:=\{10.0,10.0\}]])$;

genes([ name := "Operon2",

proteins := $\{$ "rel2", "lacI" $\}$,

promoter $:=[$ function $:=$ "TRUE"],

prot_act_times $:=[$ times $:=\{0.2,50.0\}]$,

prot_deg_times $:=[$ times $:=\{10.0,10.0\}]])$;

// Plasmid inclusion matrix (of operons)

plasmids_genes ( $[\quad \mathrm{p} 1:=\{$ "Operon1" $\}$,

p2 :=\{"Operon2" $\}]$ );

\section{//ACTIONS}

//Express "color" with a fixed saturation. Code: $\{G, R, Y, C\}$

action (\{"gfp", "-rel2"\}, "paint", \{"500", "0" , "0" , "0"\});

//Express "color" gradually. Code: $\{\mathrm{G}, \mathrm{R}, \mathrm{Y}, \mathrm{C}\}$

//action (\{"-gfp" \}, "d_paint", \{"-1", "0", "0", "0"\});

action (\{"rel2", "-gfp"\}, "paint" , \{"0" , "500", "0", "0"\});

action ( $\{" r e 12 "$, "gfp", , paint", \{"0", "0", "500", "0" $\})$;

//action (\{"gen2"\}, "paint", \{"0", "500", "0", "0"\});

//action (\{"-gfp"\}, "d_paint", \{"-20", "0", "0", "0" $\})$;

//Plasmid id, conjugation rate

//action(\{"rel1"\}, "conjugate", \{"p1", "1.0"\});

//action (\{"rel2"\}, "conjugate", \{"p2", "1.0"\});

action ( $\{$ "lacI" $\}, " d i e ")$;

//Plasmid id

//action (\{"-ara", "tetR" \}, "lose_plasmid", \{"p1"\});

$/ / \ln (2) / \mathrm{gt}(\mathrm{min})[\mathrm{um} / \mathrm{min}]$

//action(\{"gfp"\}, "set_growth_rate", \{"0.011"\});

//Cross-feeding emission tag, concentration, emission mode

//action (\{"gen1"\}, "s_emit_cf", \{tostring (cf1), "20" , "exact"\});

//action (\{"gen2"\}, "s_emit_cf", \{tostring (cf2), "20" , "exact"\});

//Cross-feeding reception tag, concentration, benefit: 1. good, -1. toxin

//action (\{"gen1"\}, "get_cf", \{tostring (cf2), "20", "-1"\});

//action (\{"gen2"\}, "get_cf", \{tostring (cf1), "20", "-1"\}); 


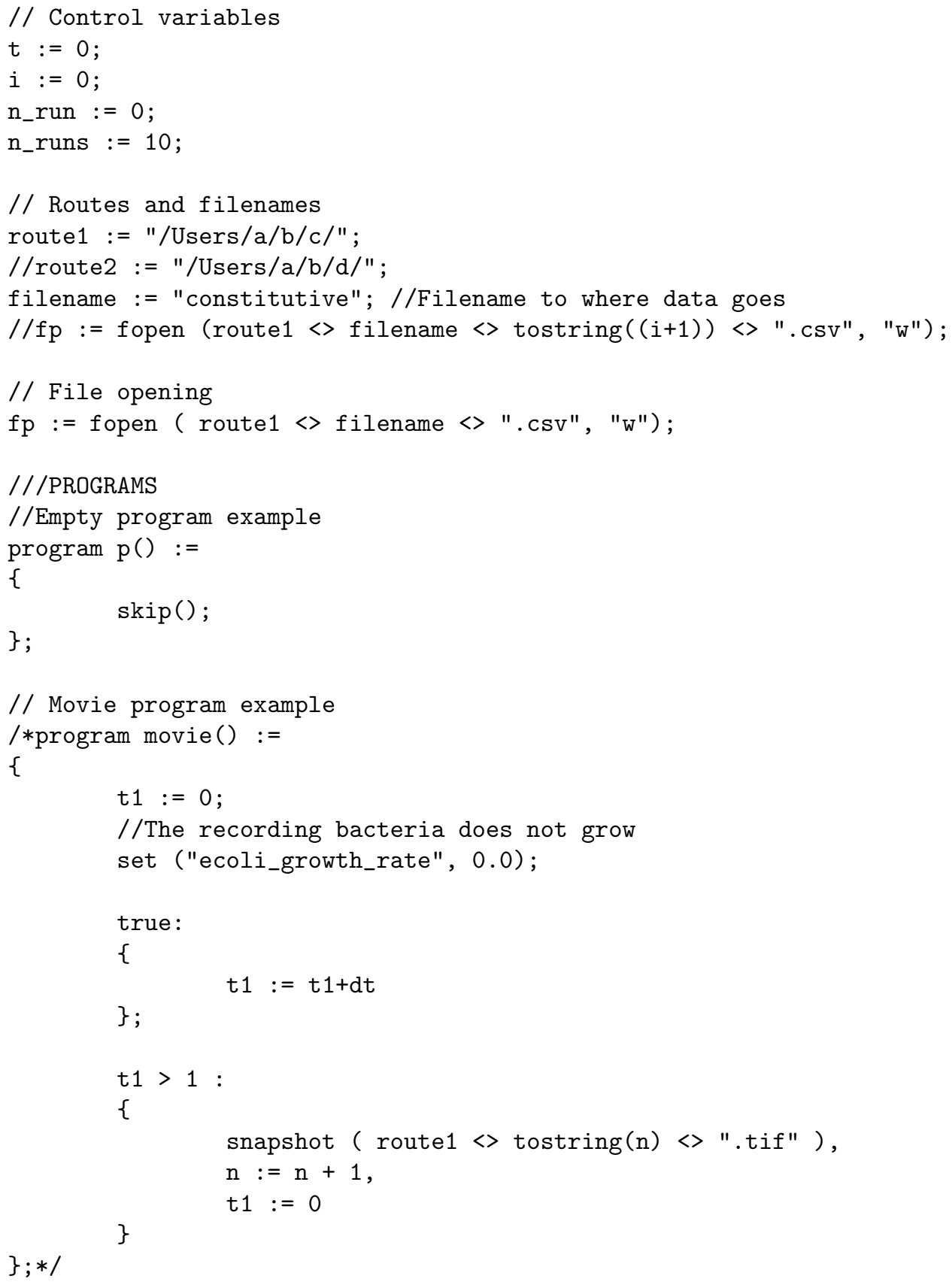




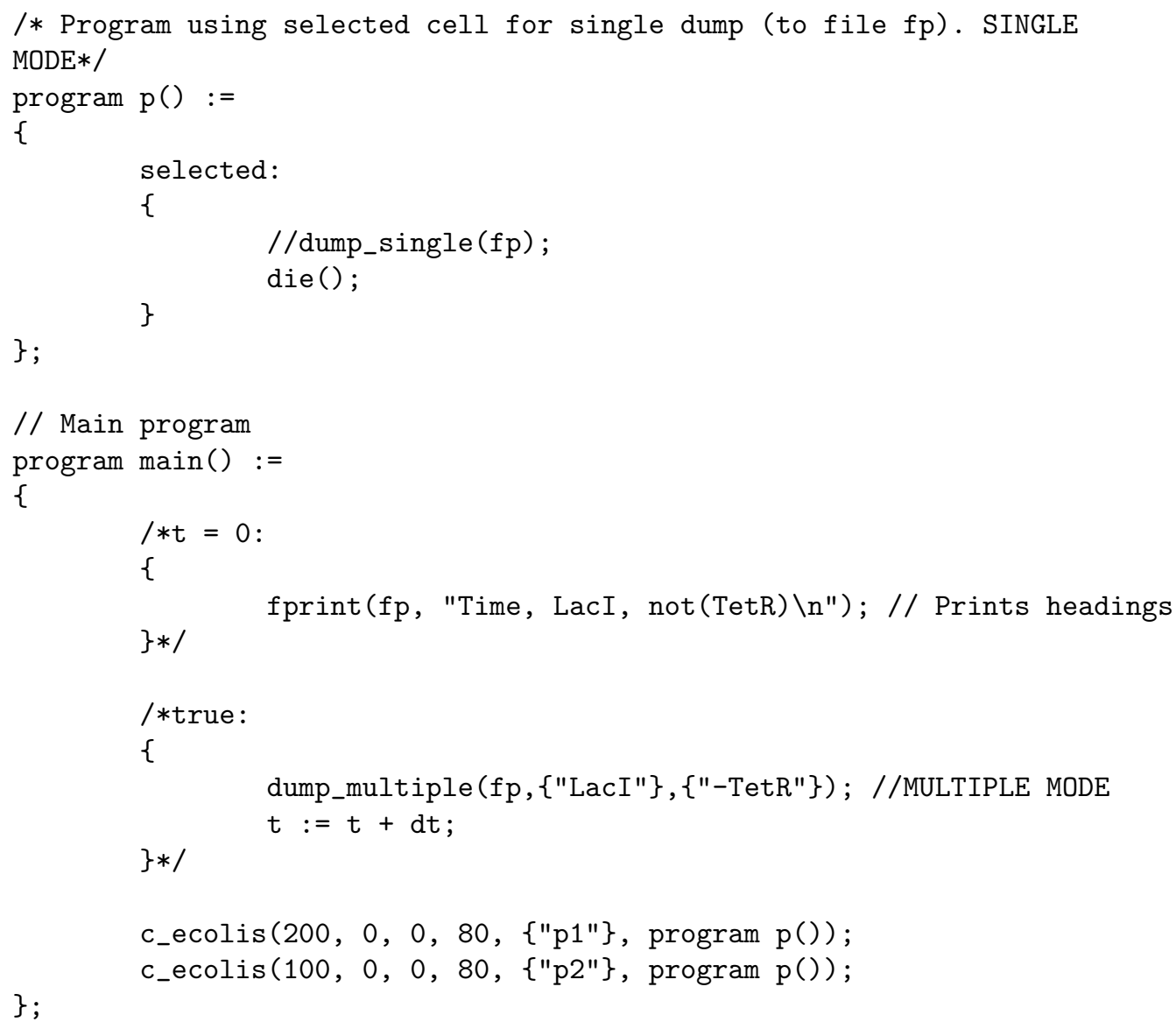

More examples can be found at https://github.com/liaupm/GRO-LIA in the "PaperExamples" folder. 


\section{Appendix B}

\section{-gro sources}

This appendix concentrates all of the .gro source codes used for simulating designs in this document. All of the parameters used in the simulations can be found here.

\section{B.1 YES Gate specification with guarded com- mands}

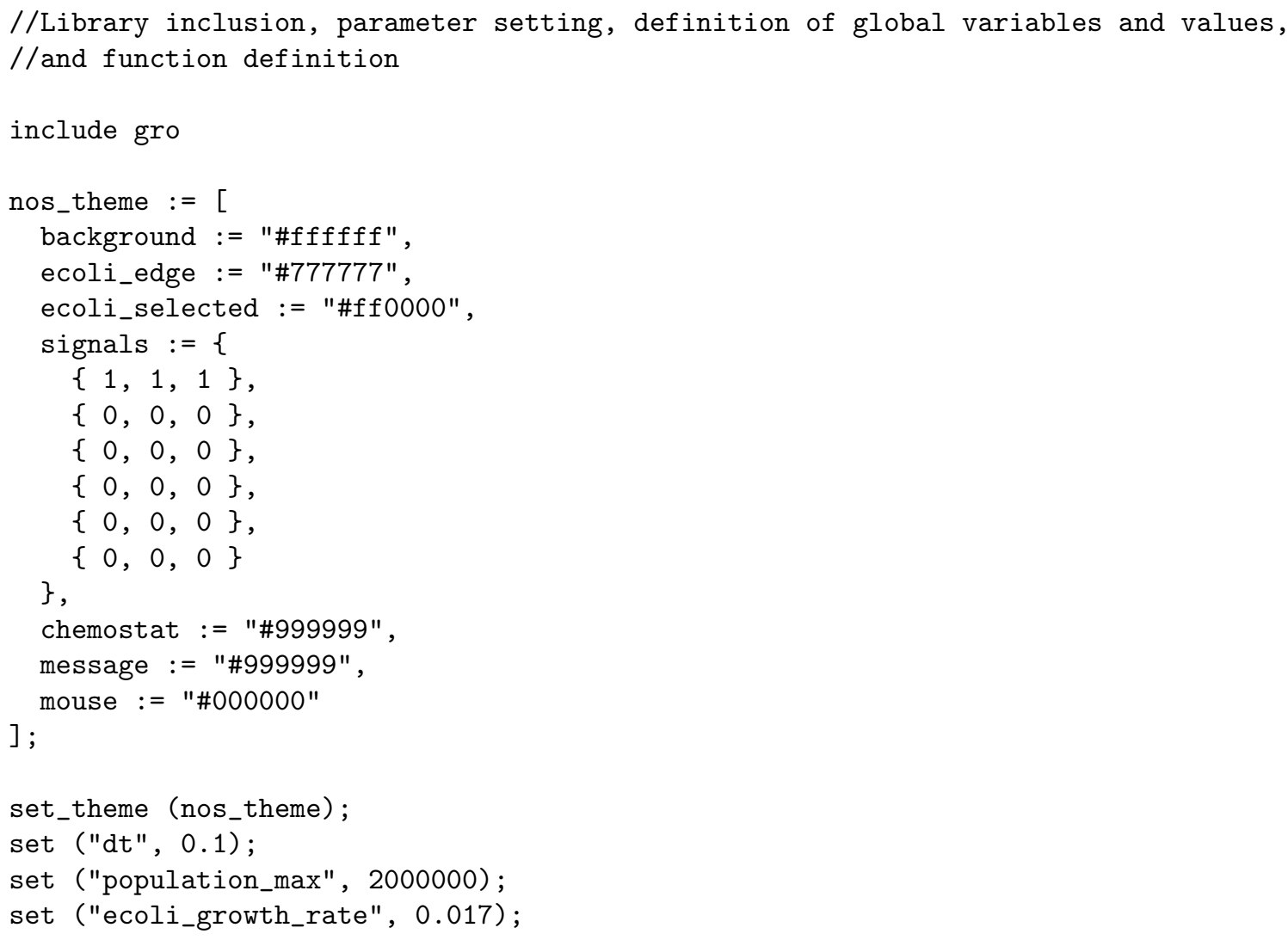




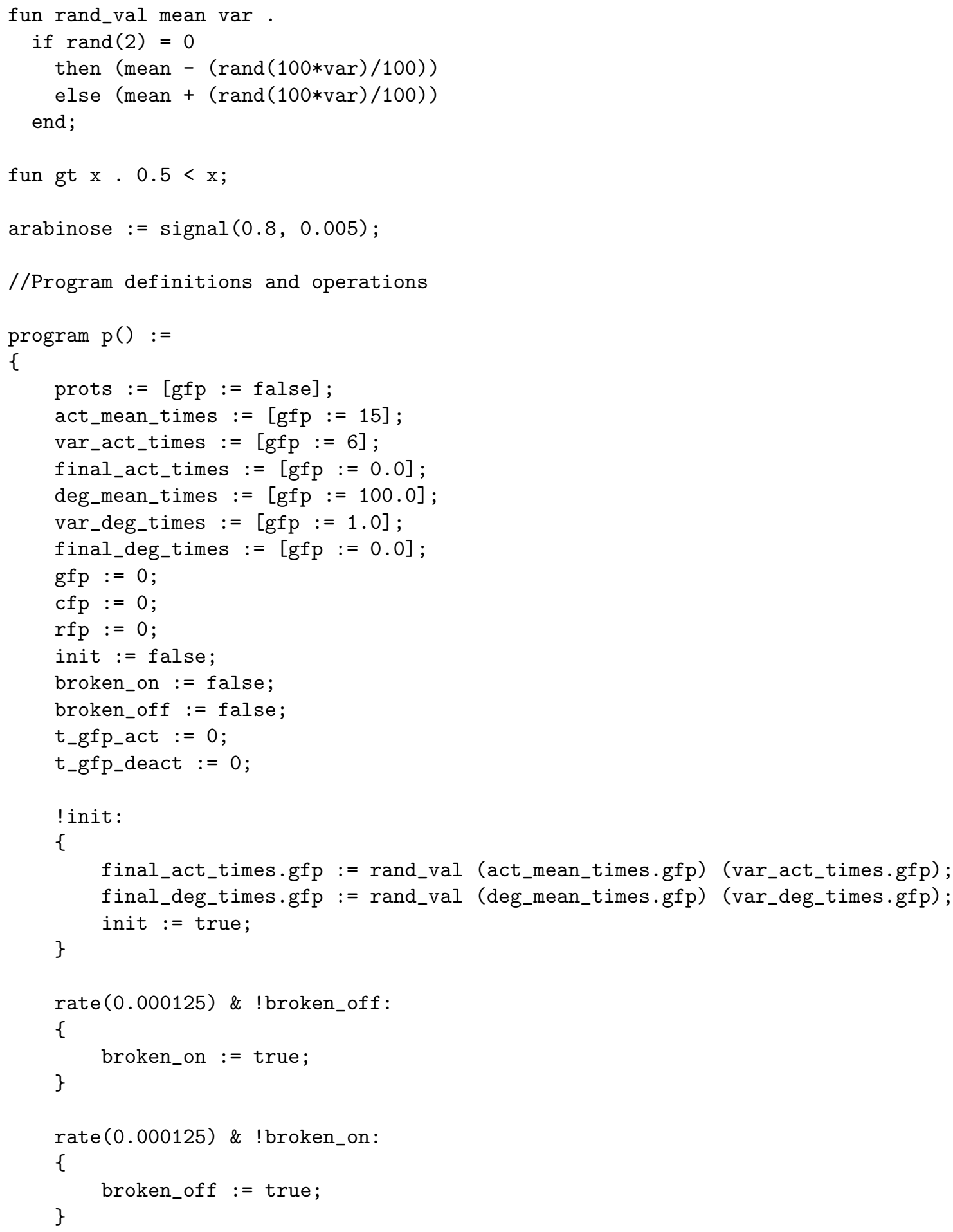




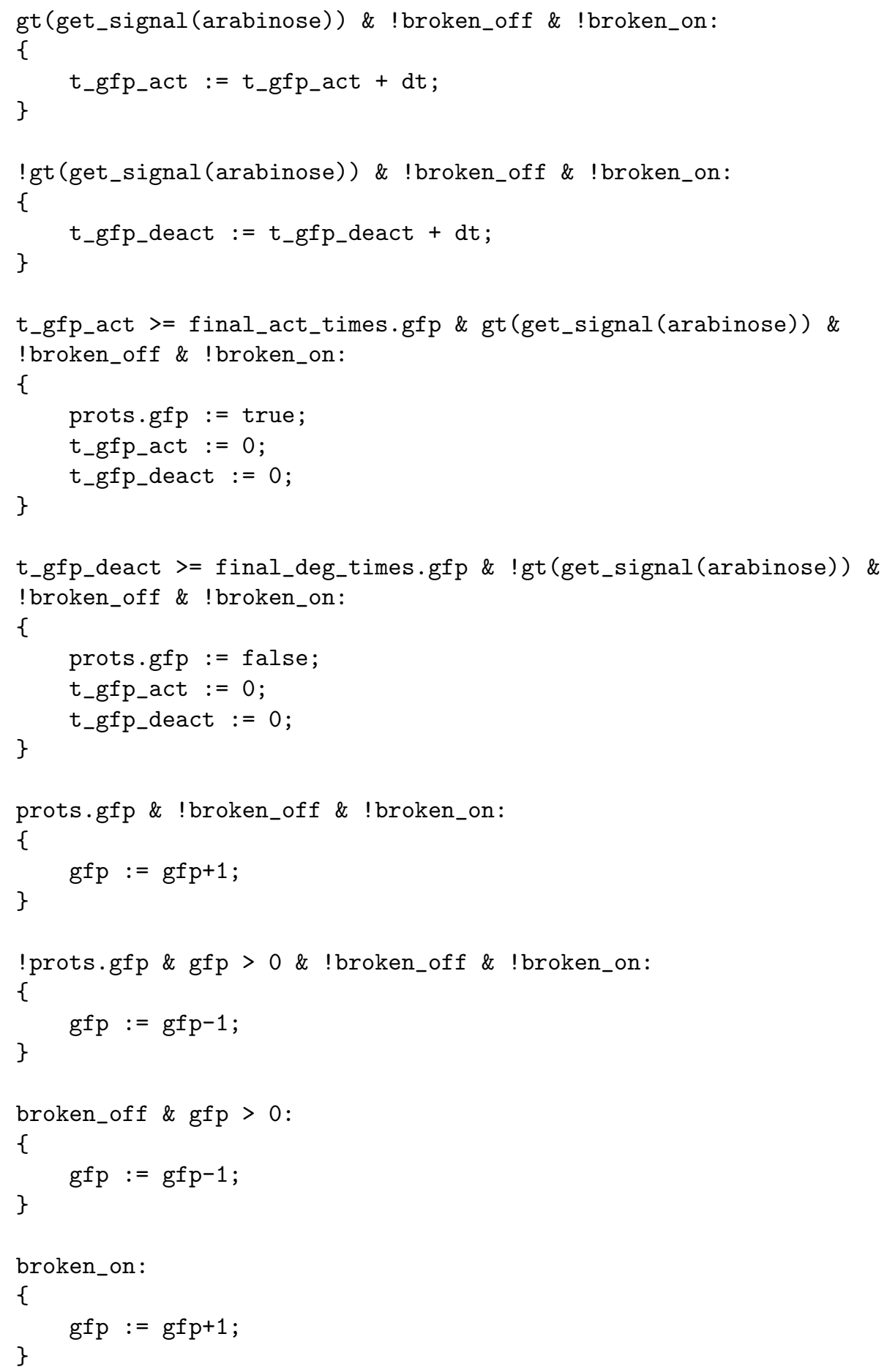




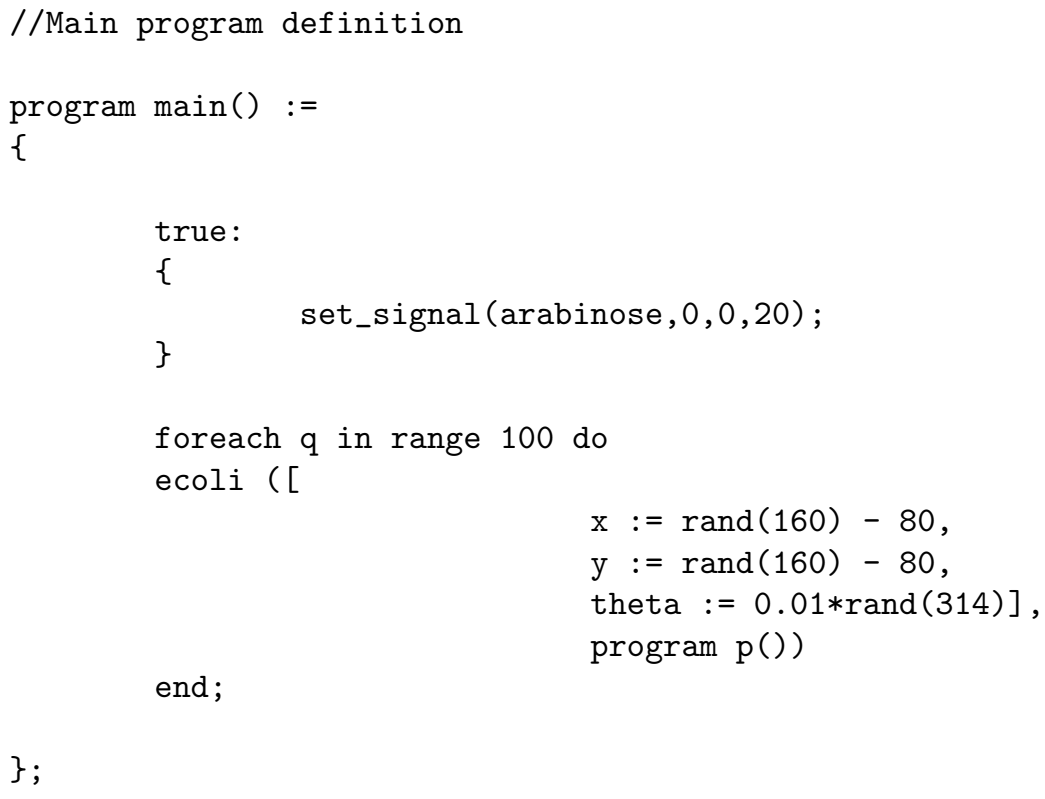




\section{B.2 YES Gate specification in ProSpec}

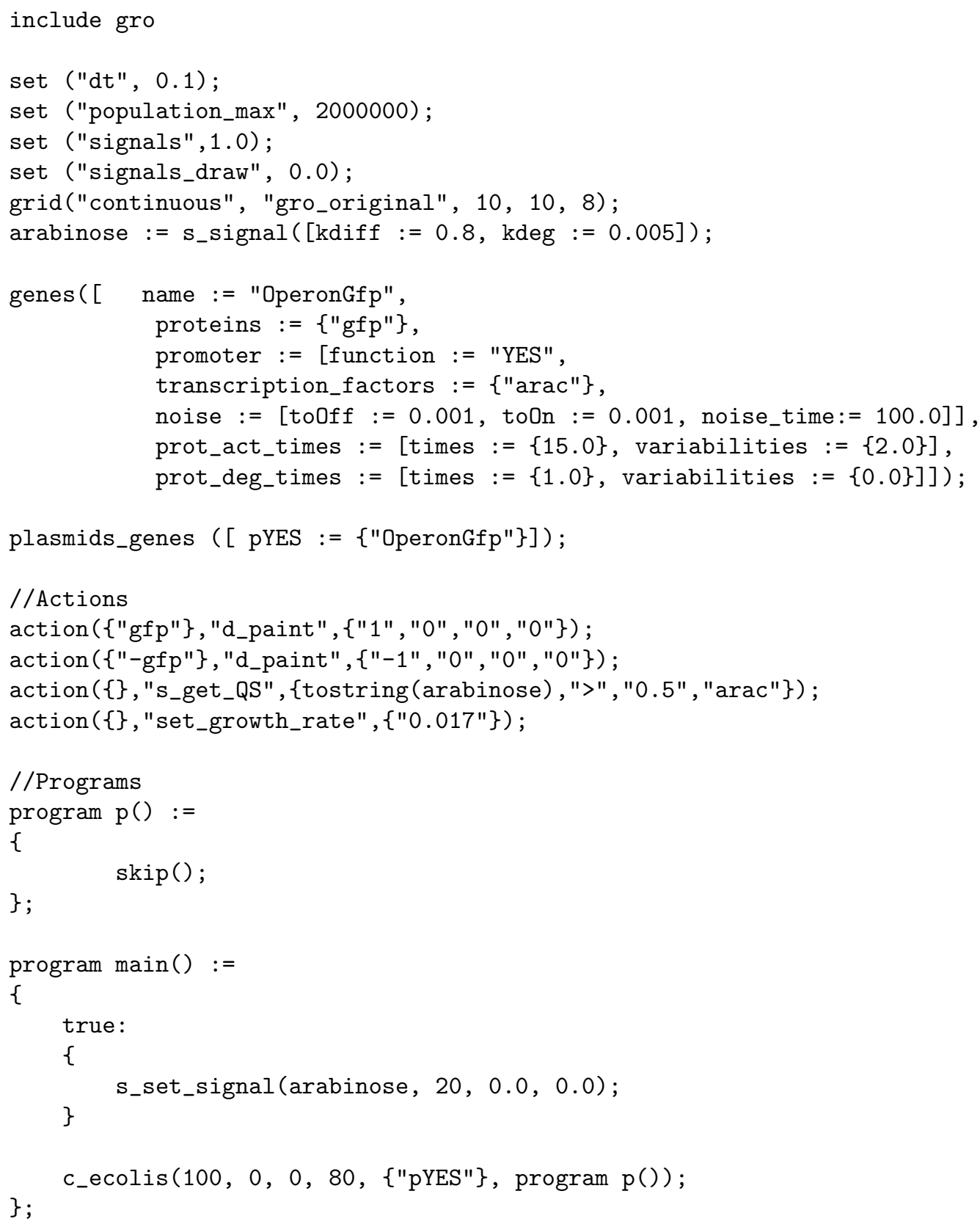




\section{B.3 Two-level bullseye pattern}

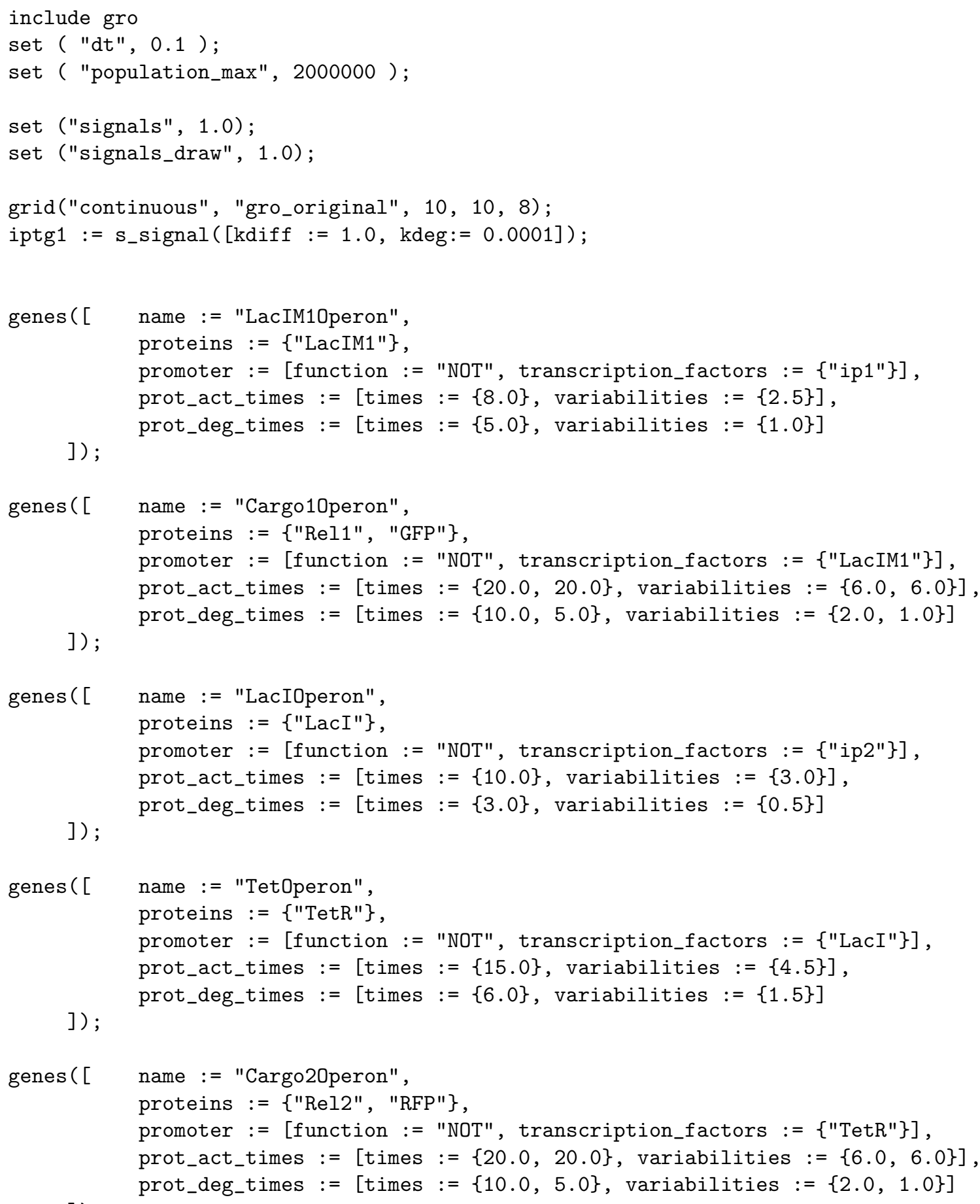




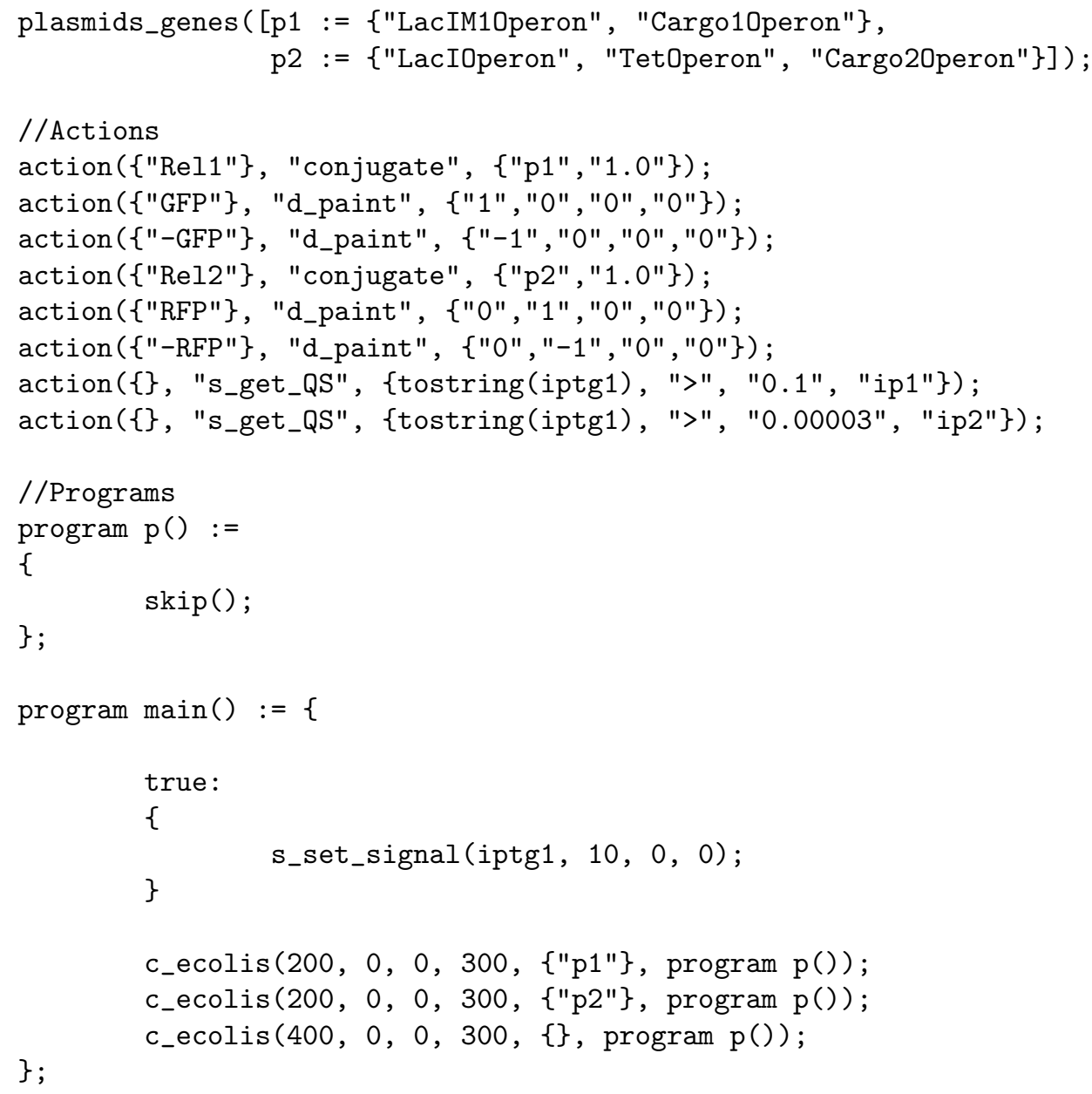




\section{B.4 Three-level bullseye pattern}

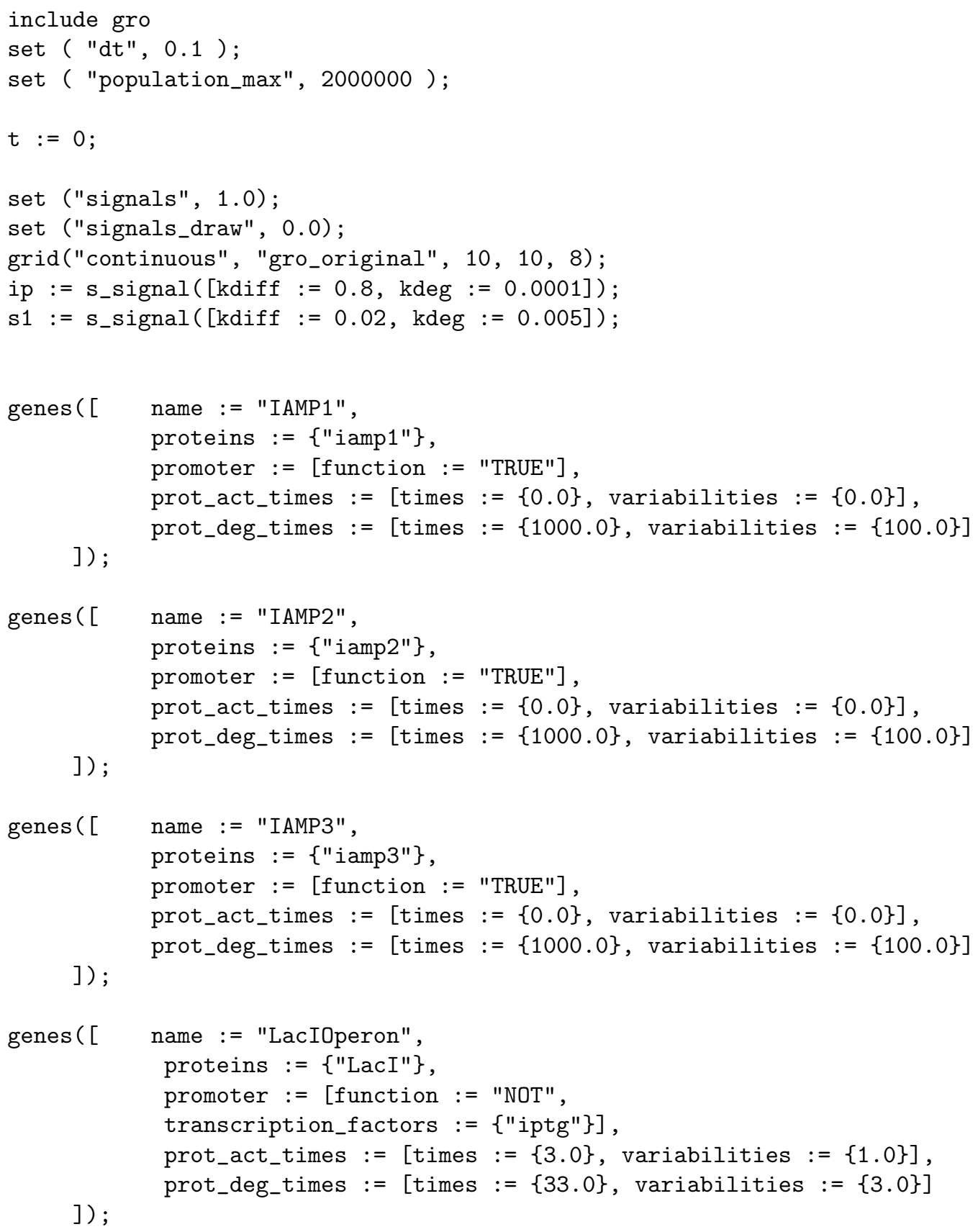




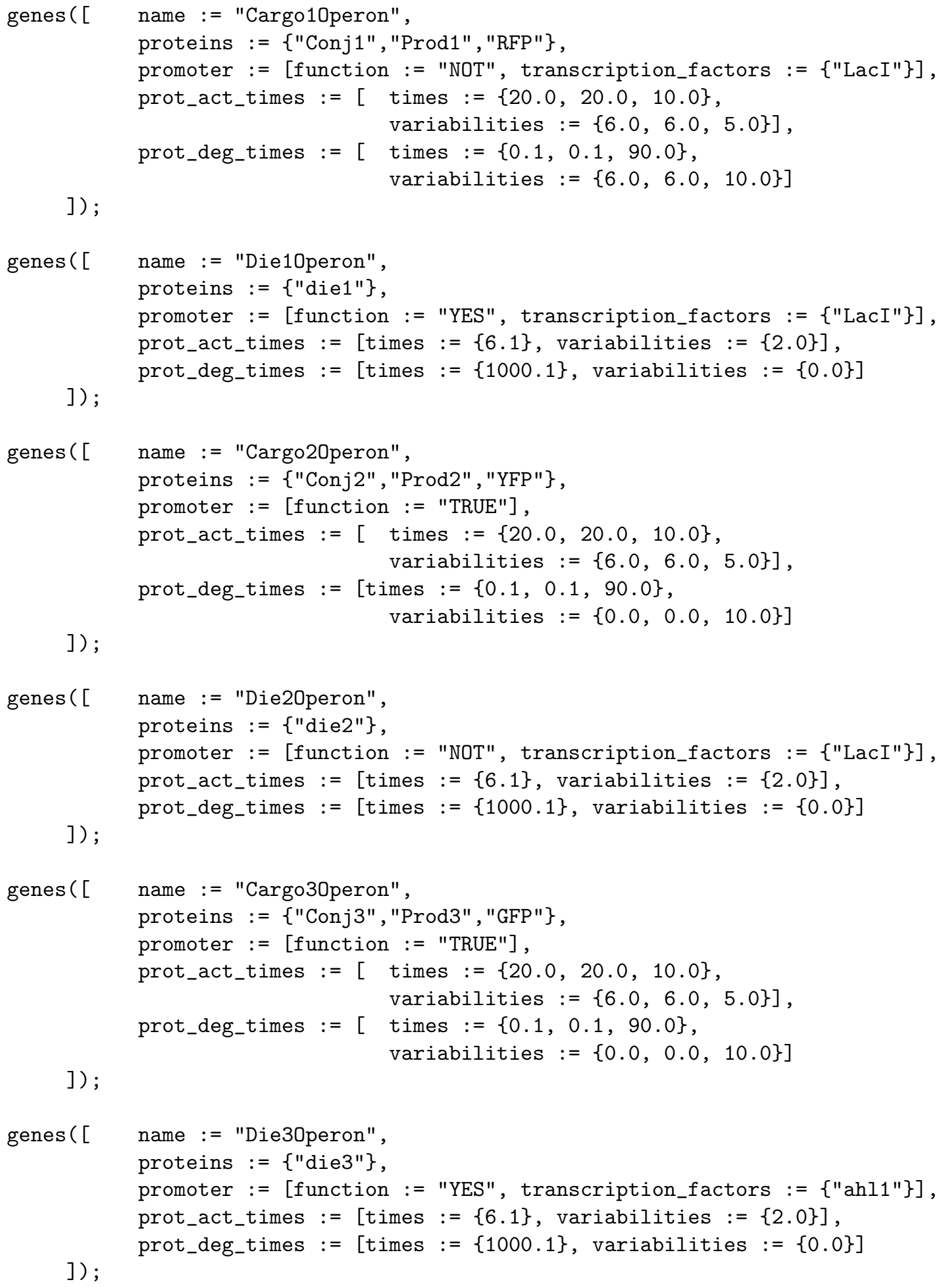




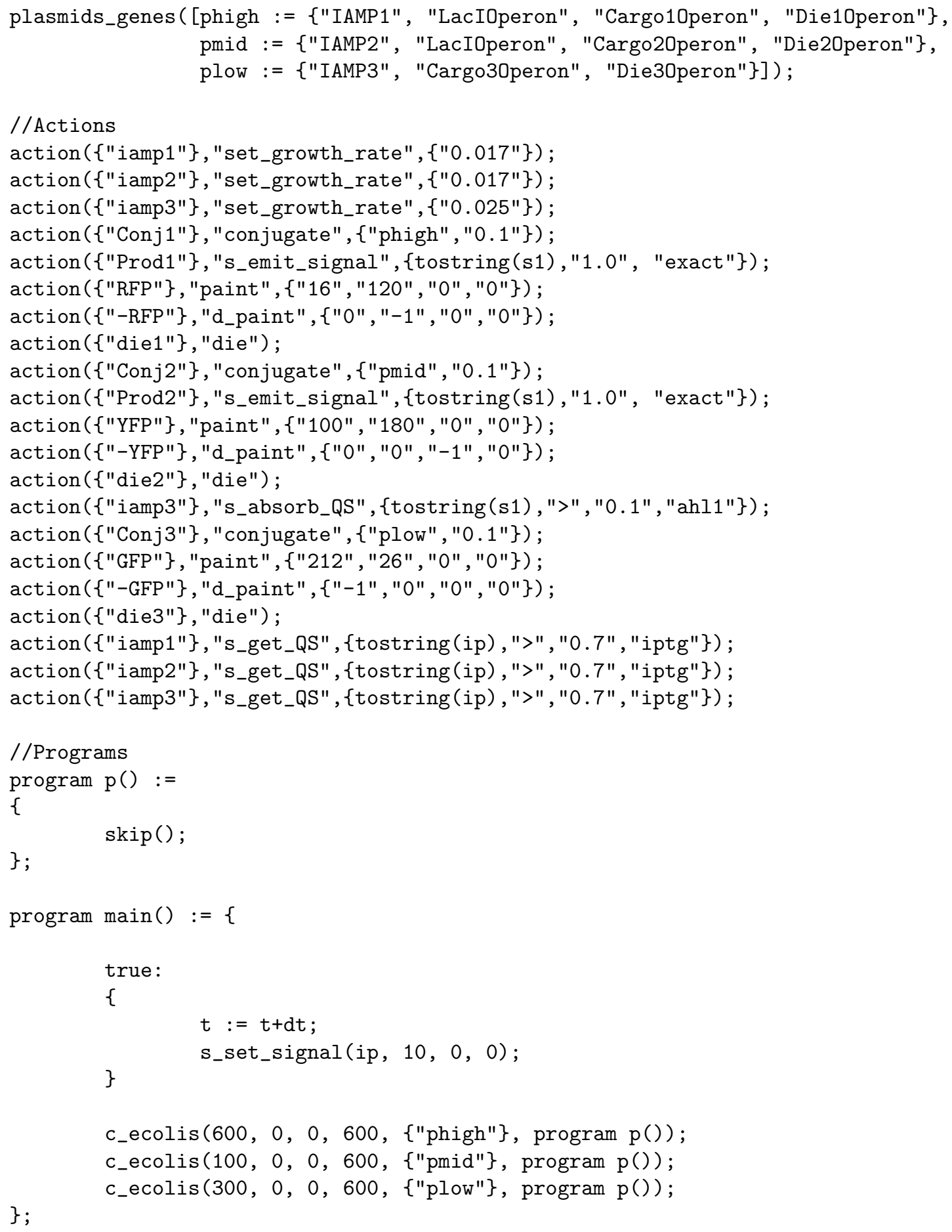




\section{B.5 Four-level bullseye pattern}

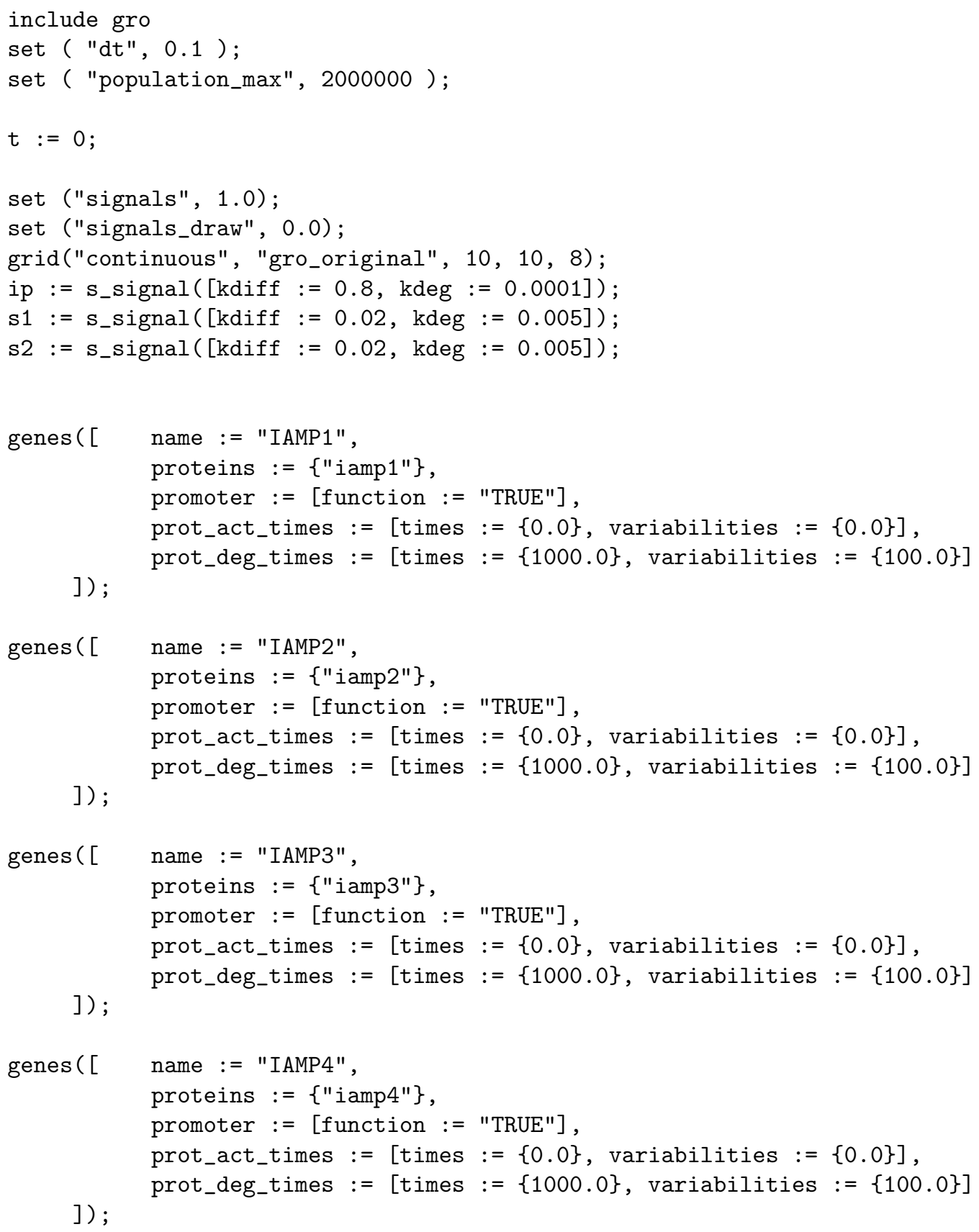




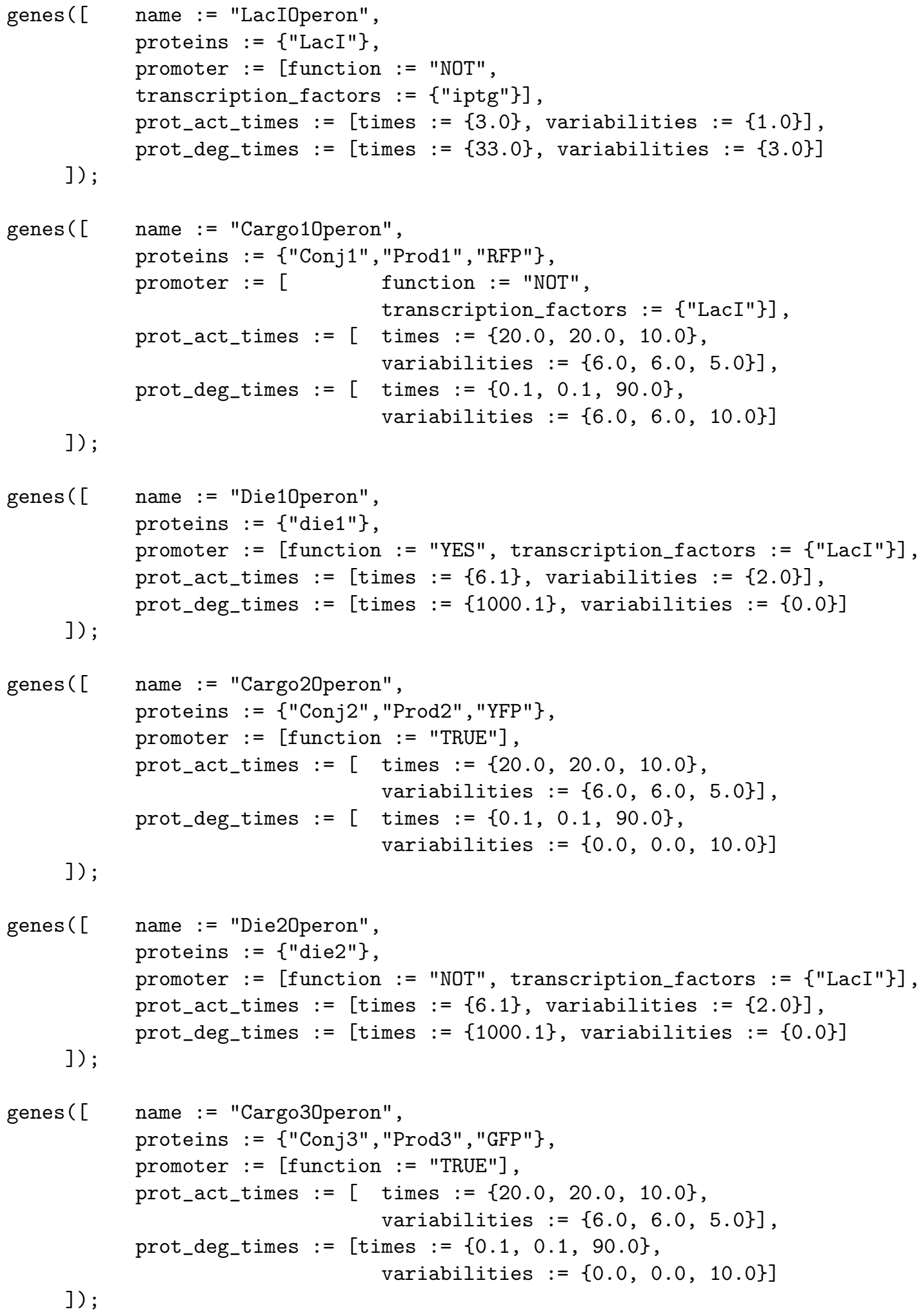

]); 


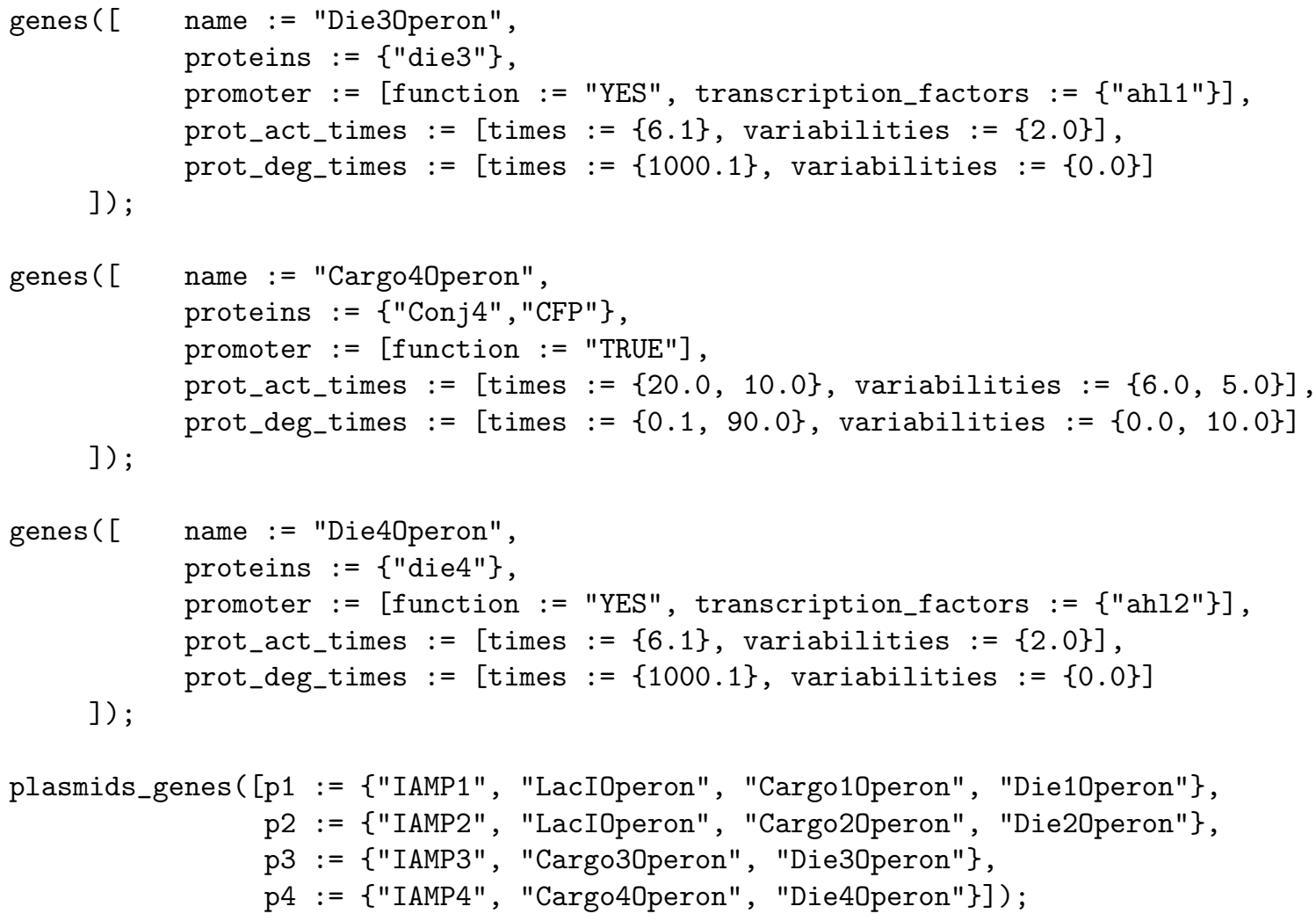

//Actions

action (\{"iamp1"\}, "set_growth_rate", \{"0.017"\});

action ( $\{$ "iamp2" $\}$, "set_growth_rate", \{"0.017"\});

action (\{"iamp3"\}, "set_growth_rate", $\{" 0.017 "\})$;

action (\{"iamp3"\}, "set_growth_rate", \{"0.034"\});

action ( $\{$ "Conj 1"\}, "conjugate", \{"p1", "0.1"\});

action (\{"Prod1"\},"s_emit_signal", \{tostring(s1),"1.0", "exact"\});

action (\{"RFP" $\}$, "paint", \{"16", "120", "0", "0"\});

action (\{"-RFP" $\},$ "d_paint", \{"0", "-1", "0", "0" $\})$;

action ( $\{$ "die1"\}, "die");

action (\{"Conj2"\}, "conjugate", \{"p2", "0.1"\});

action (\{"Prod2"\},"s_emit_signal", \{tostring(s1),"1.0", "exact"\});

action(\{"Prod2"\},"s_emit_signal",\{tostring(s2),"1.0", "exact"\});

action (\{"YFP" $\},$ "paint" , \{"100", "180" , "0" , "0" $\})$;

action (\{"-YFP" $\},$ "d_paint", \{"0", "0" , "-1", "0" $\})$;

action ( $\{$ "die2" $\}, " d i e ")$;

action (\{"iamp3"\}, "s_absorb_QS", \{tostring (s1), ">" , "0.1" , "ahl1"\});

action ( $\{$ "Conj3"\}, "conjugate", \{"p3", "0.1"\});

action(\{"Prod3"\},"s_emit_signal",\{tostring(s2),"1.0", "exact"\});

action (\{"GFP" $\}$, "paint", \{"212", "26", "0", "0" $\})$;

action (\{"-GFP" $\},$ "d_paint", \{"-1", "0" , "0" , "0" $\})$;

action ( $\{$ die3" $\}$, "die");

action( $\{$ "iamp4"\}, "s_absorb_QS", \{tostring(s2), ">", "0.1" , "ahl2"\}) ; 


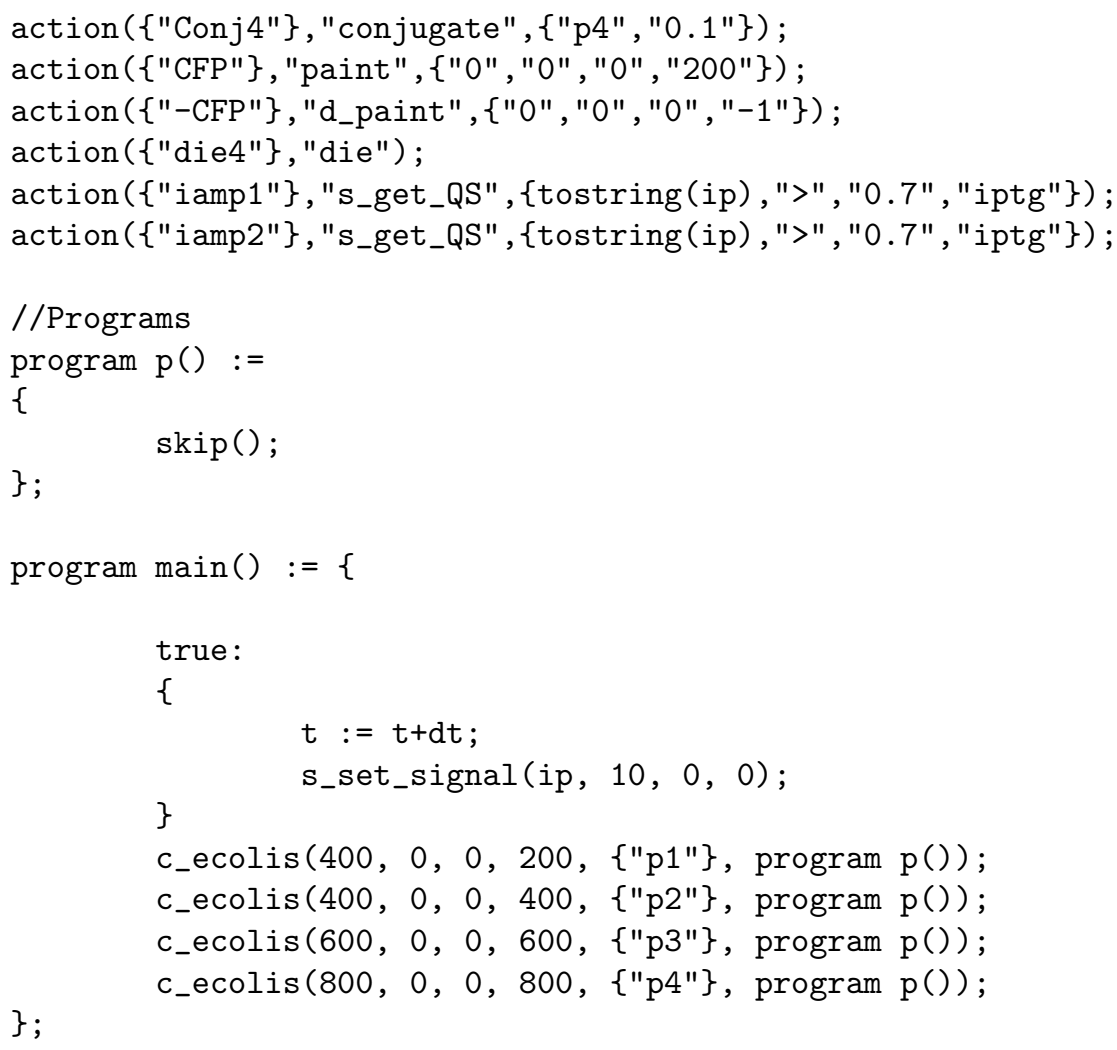




\section{B.6 Spatial oscillator with no initiating switch, variability or noise}

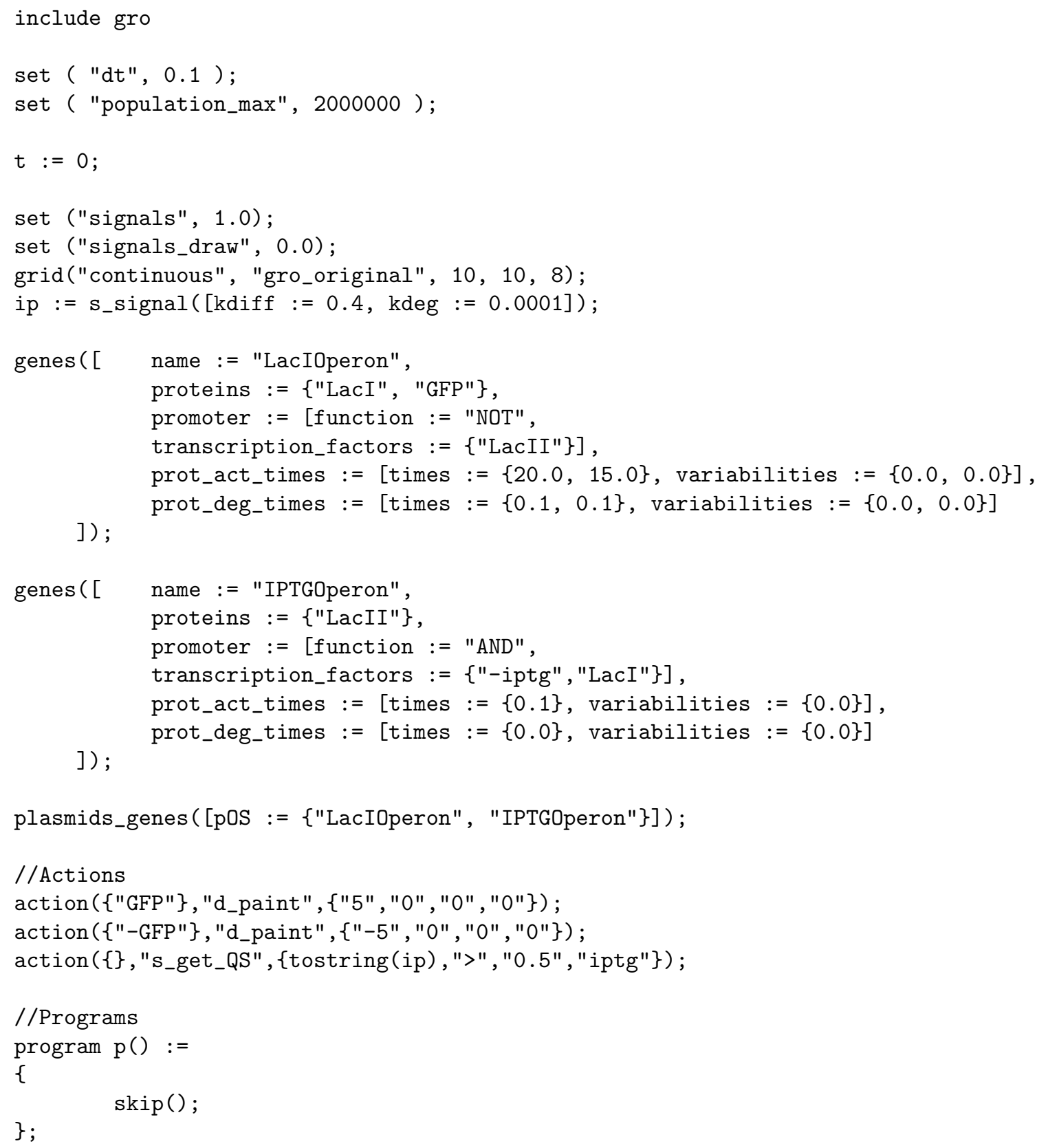




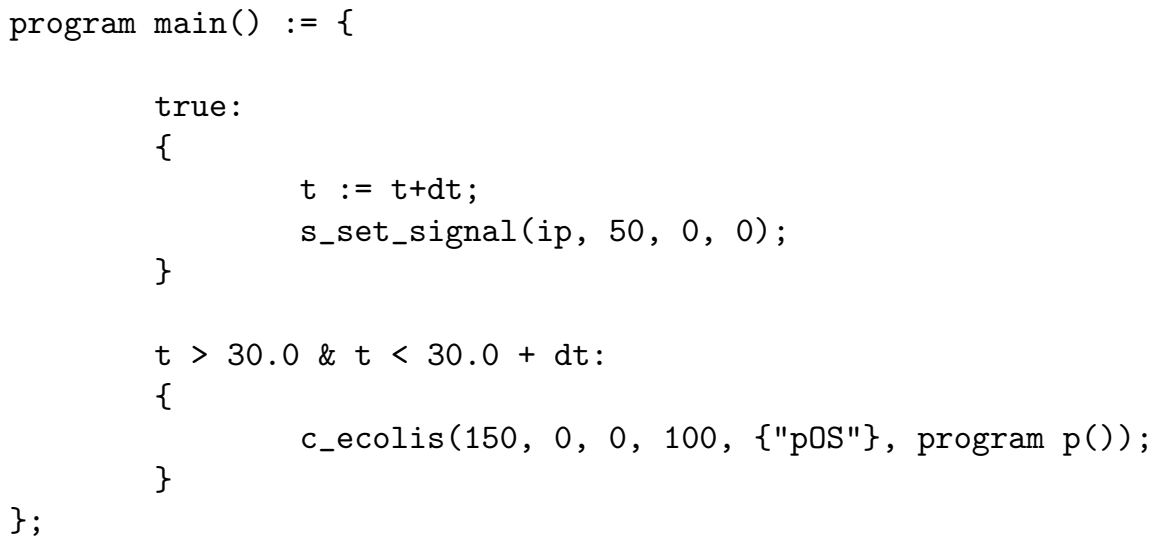




\section{B.7 Spatial oscillator with initiating switch but no variability or noise}

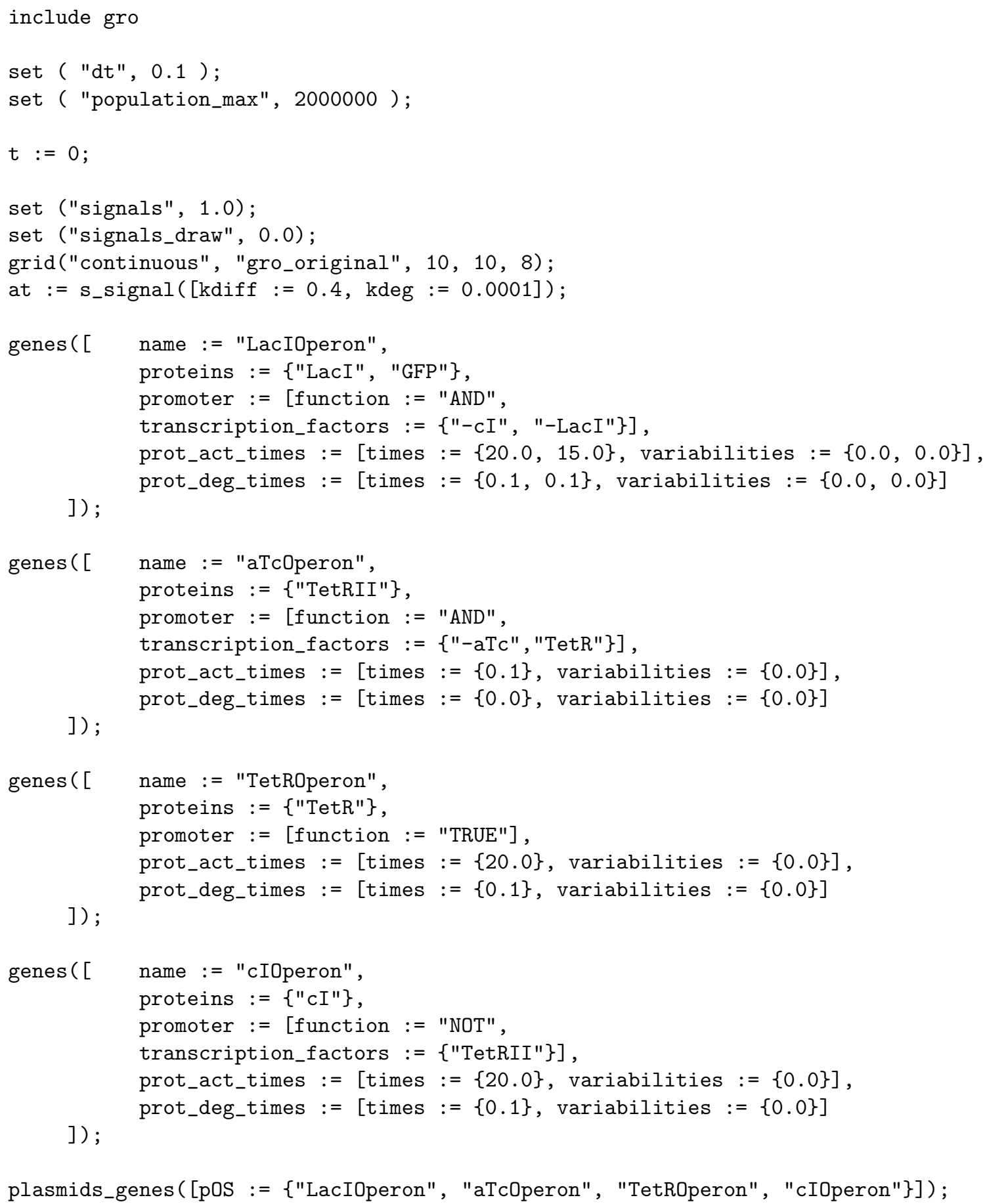




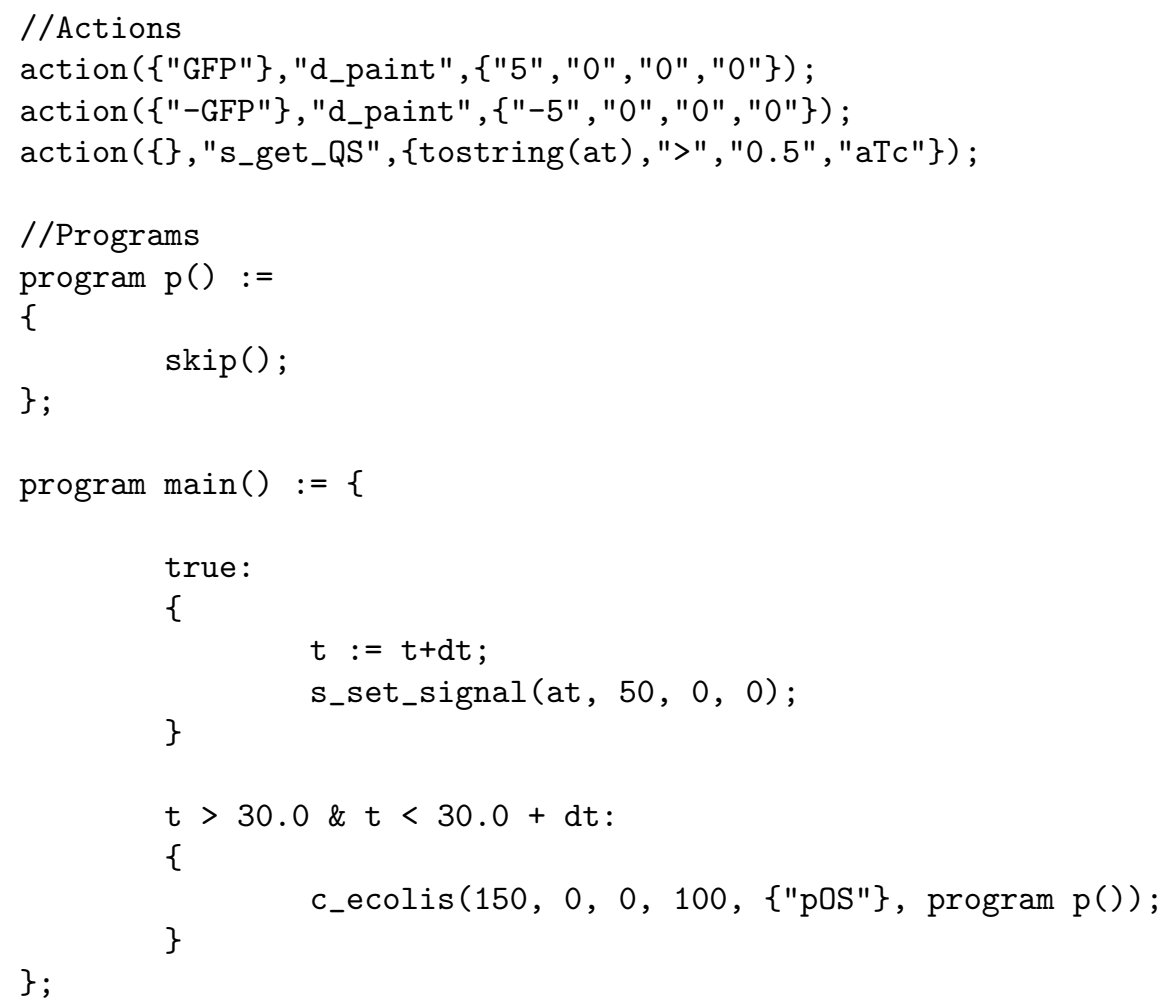




\section{B.8 Spatial oscillator with no initiating switch having variability and noise}

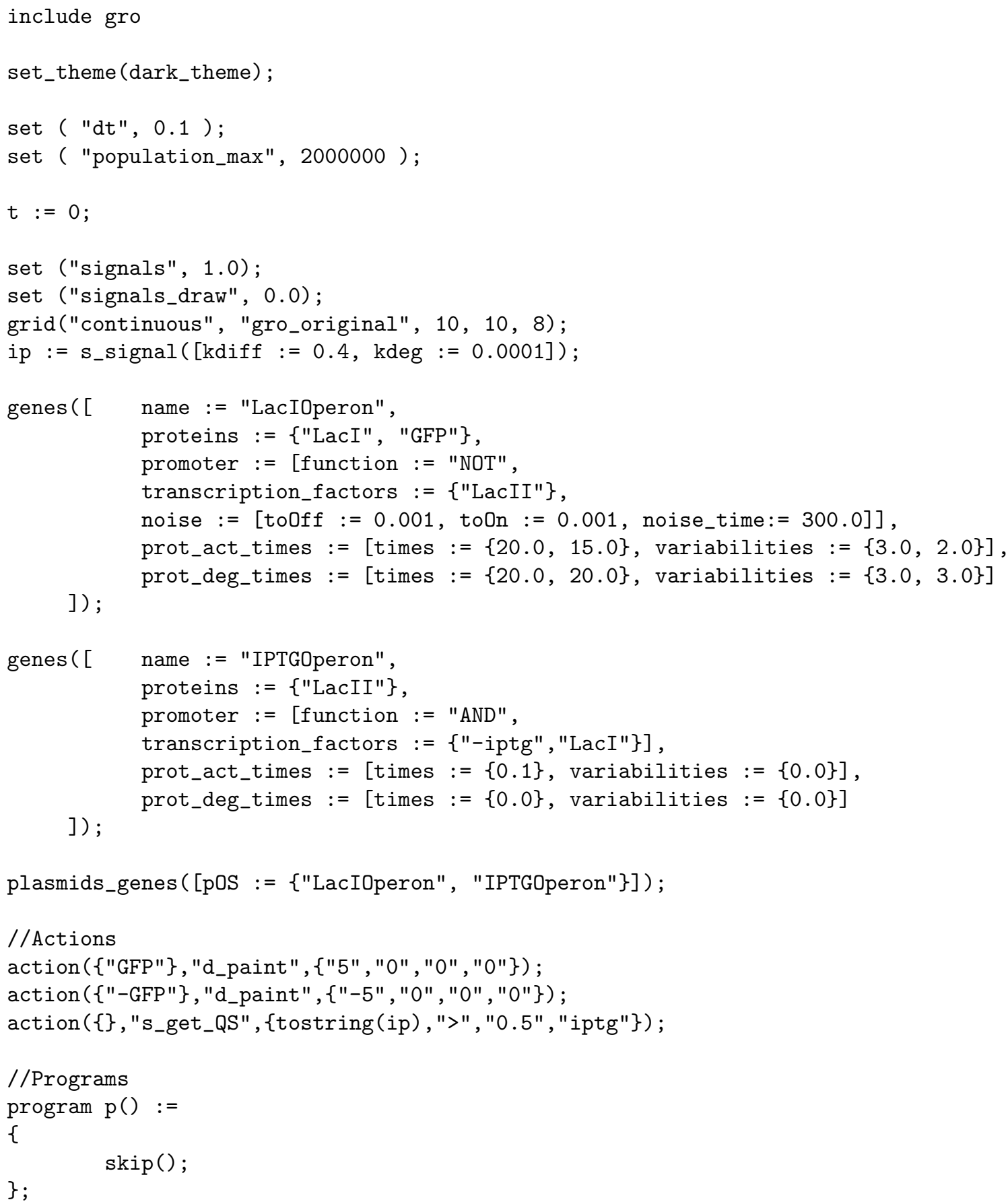




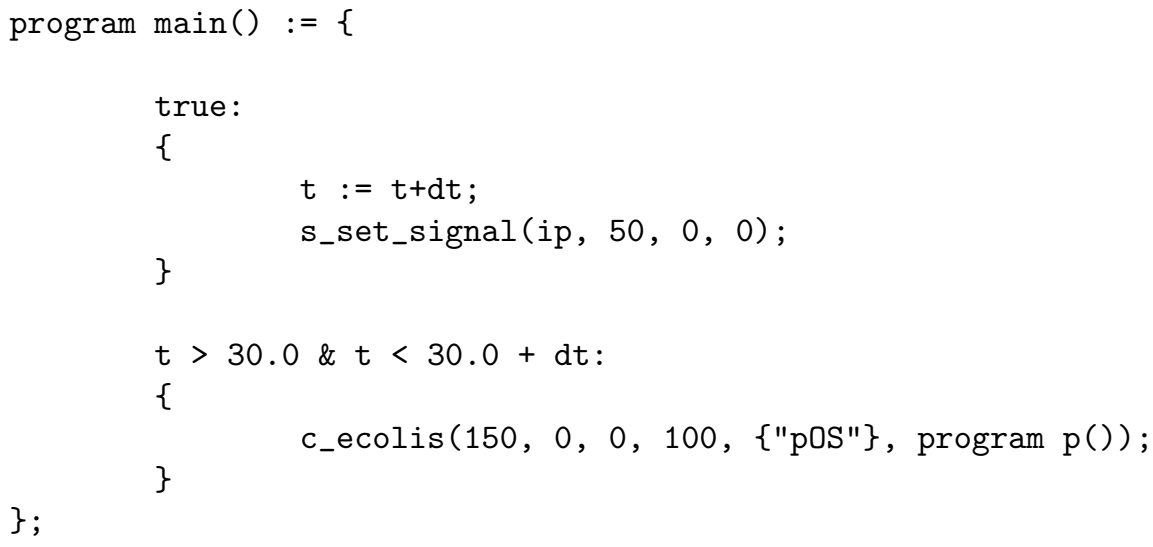




\section{B.9 Spatial oscillator with initiating switch hav- ing variability and noise}

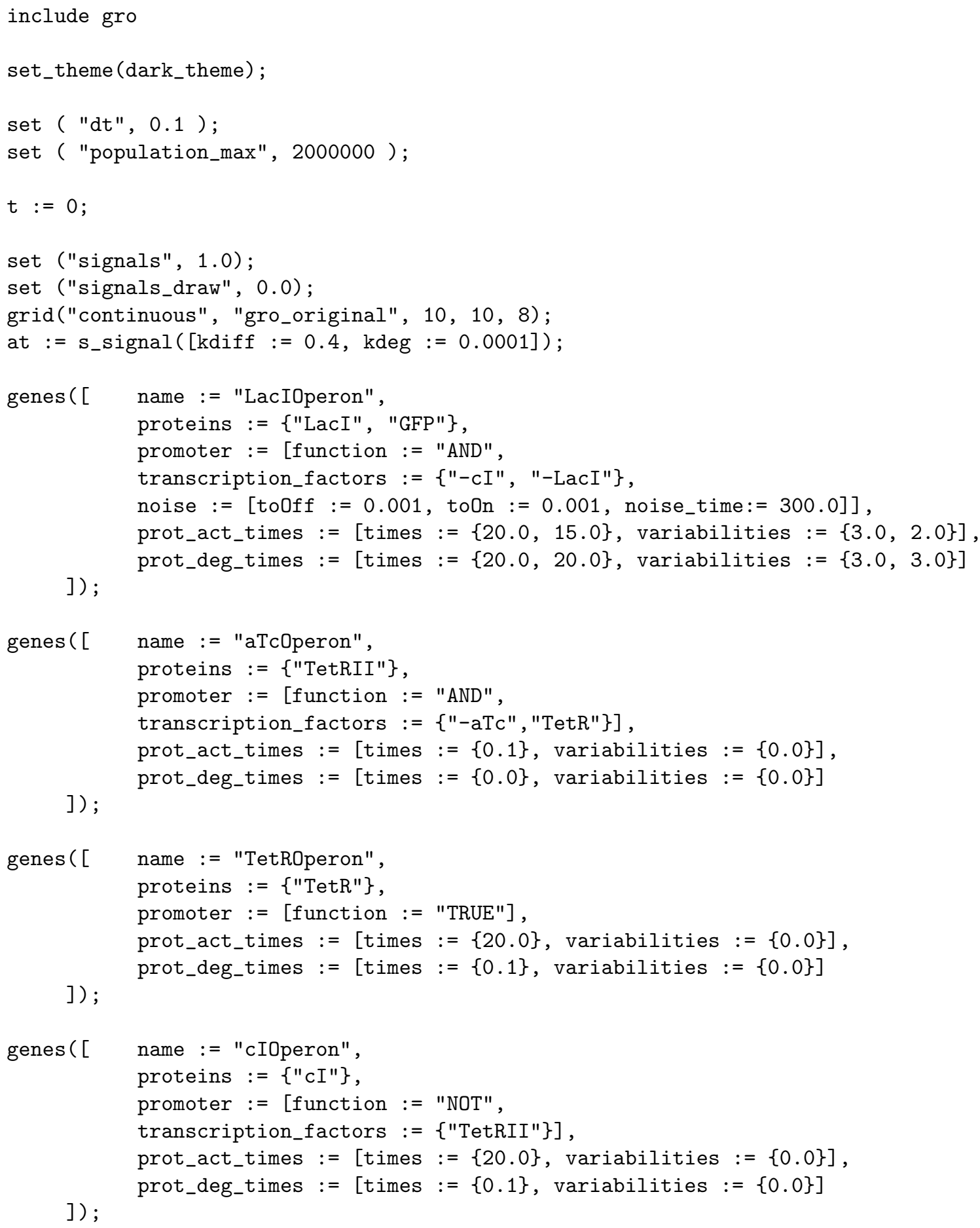




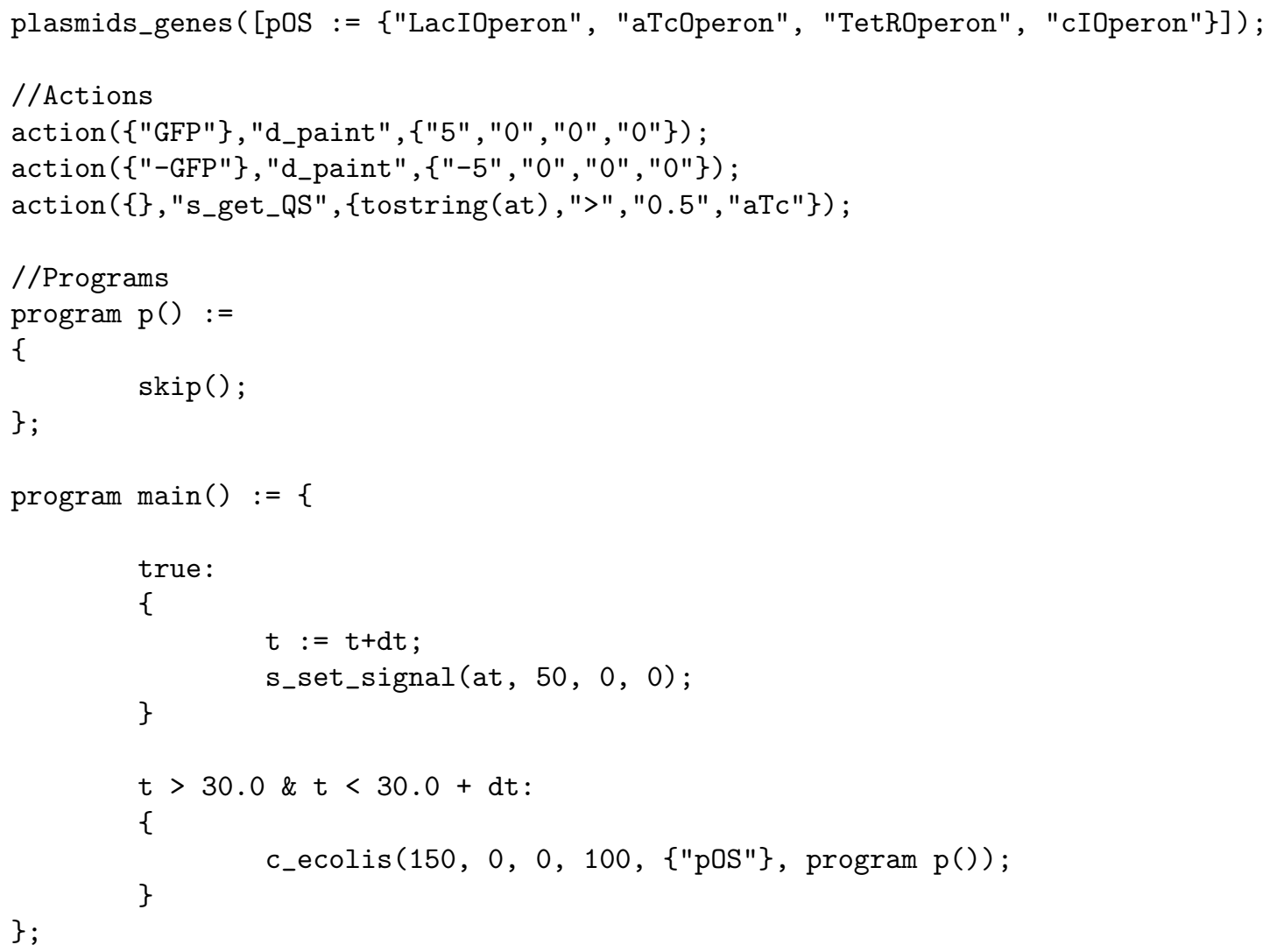




\section{B.10 Nutrient generated sun pattern}

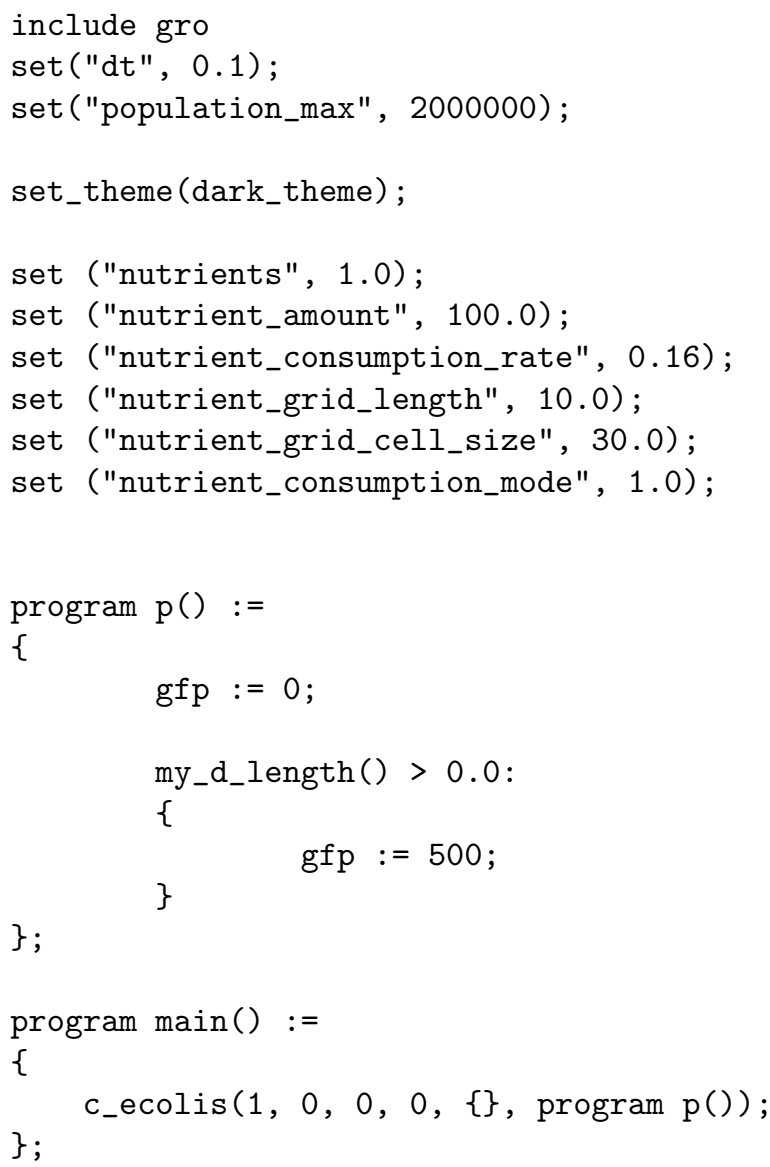




\section{B.11 Synthetic generated sun pattern}

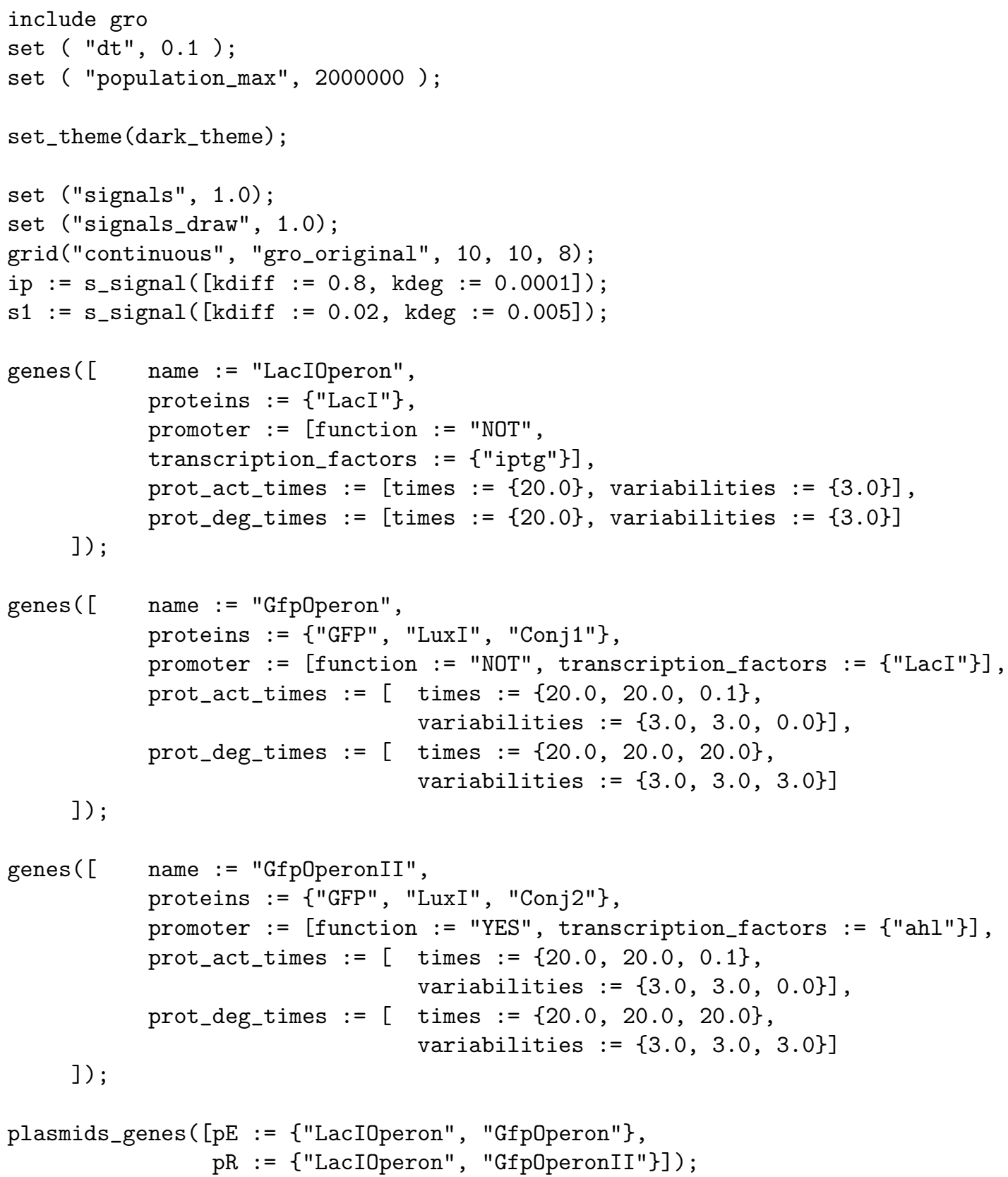




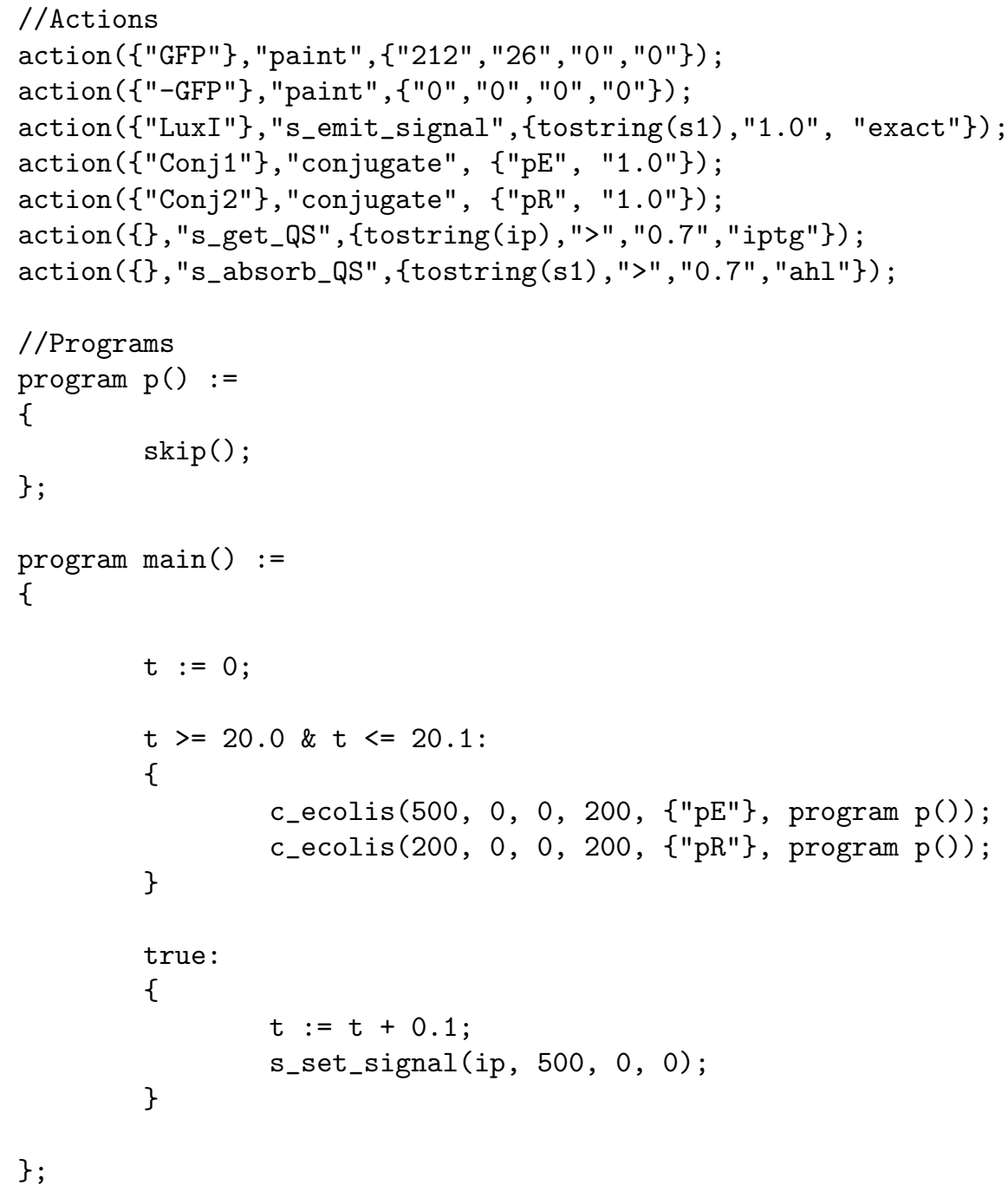




\section{B.12 Autonomous bioreactor}

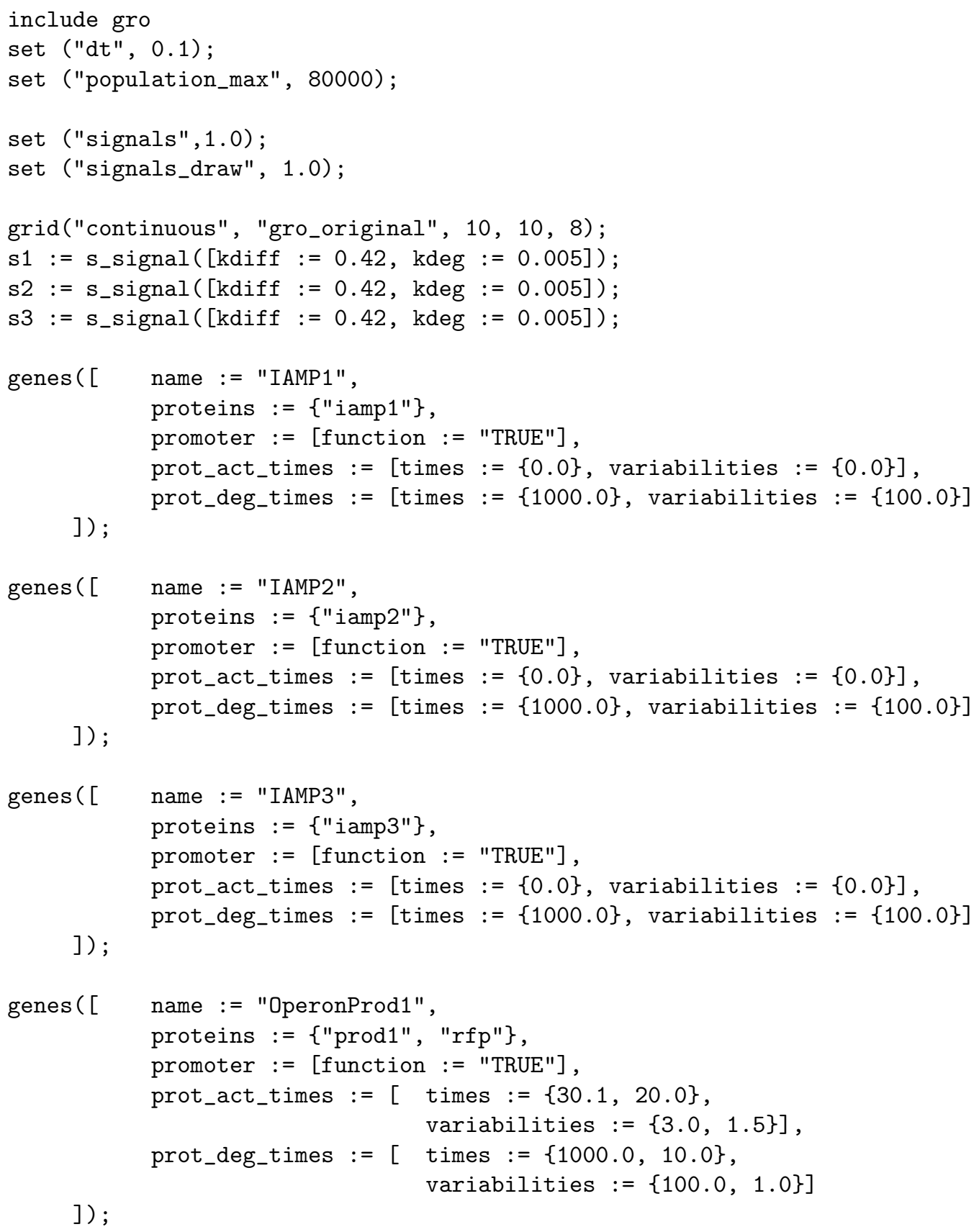




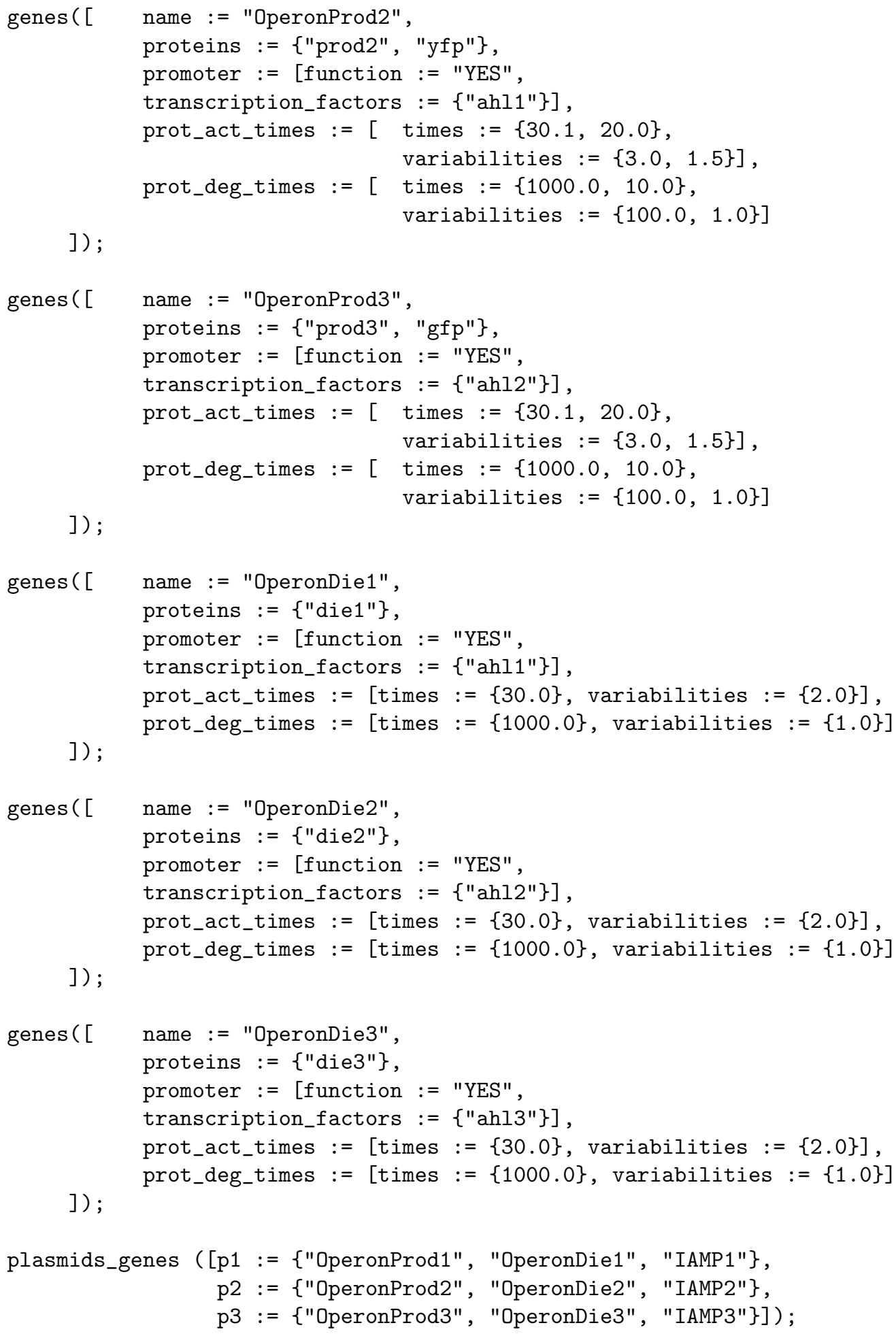




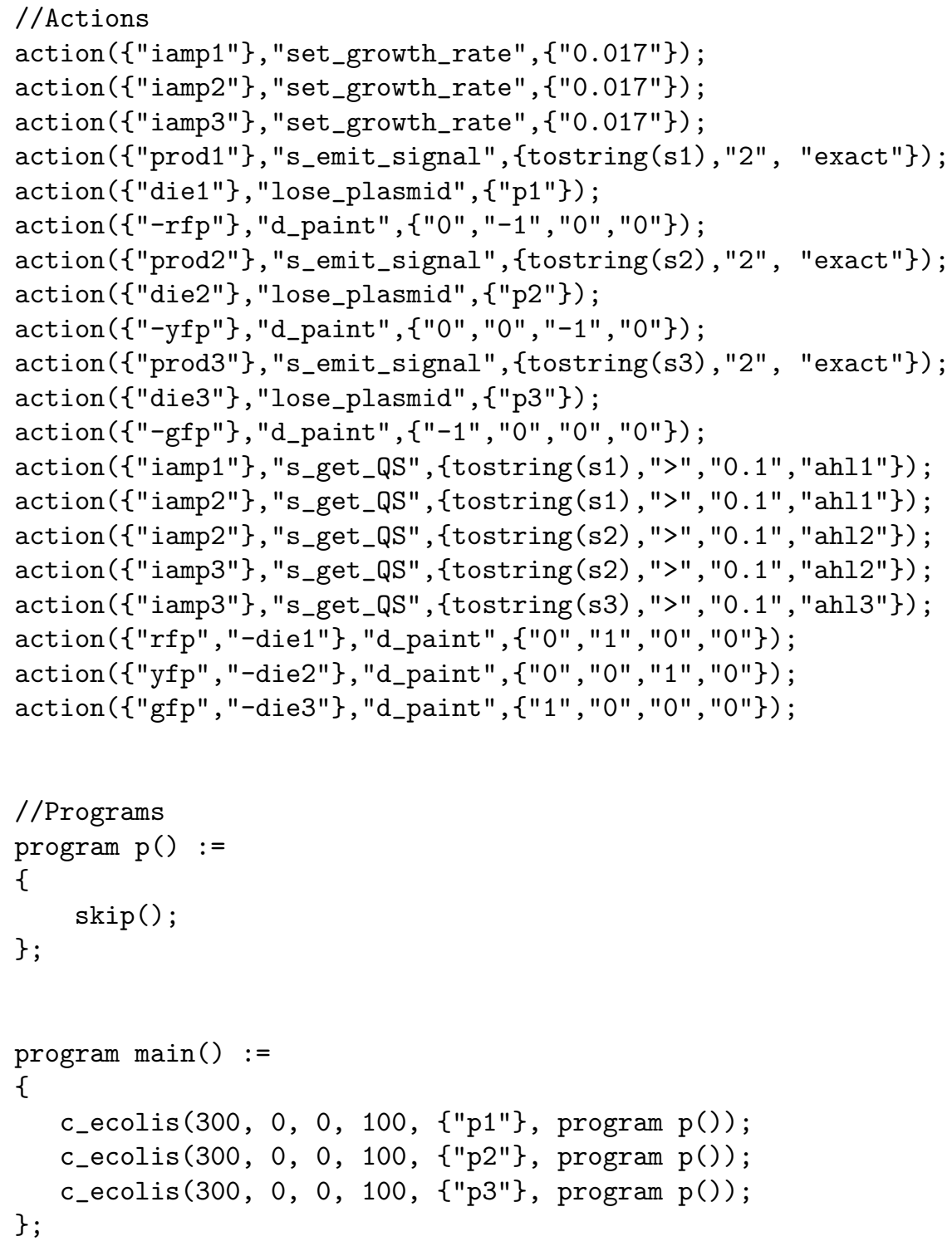

